\title{
HARMONICITY OF QUASICONFORMAL MEASURES AND POISSON BOUNDARIES OF HYPERBOLIC SPACES
}

\author{
CHRIS CONNELL ${ }^{\dagger}$ AND ROMAN MUCHNIK ${ }^{\ddagger}$
}

\begin{abstract}
We consider a group $\Gamma$ of isometries acting on a (not necessarily geodesic) $\delta$-hyperbolic space and possessing a radial limit set of full measure within its limit set. For any continuous $\alpha$-quasiconformal measure $\nu$ supported on the limit set, we produce a stationary measure $\mu$ on $\Gamma$. Moreover the limit set together with $\nu$ forms a $\mu$-boundary and $\nu$ is harmonic with respect to the random walk induced by $\mu$. In the case when $X$ is a $\operatorname{CAT}(-\kappa)$ space and $\Gamma$ acts cocompactly, for instance, we show that $\mu$ has finite first moment. This implies that $(\partial X, \nu)$ is the unique Poisson boundary for $\mu$. In the course of the proofs, we establish sufficient conditions for a set of continuous functions to form a positive basis, either in the $L^{1}$ or $L^{\infty}$ norm, for the space of uniformly positive lower-semicontinuous functions on a metric measure space.
\end{abstract}

On the hyperbolic plane $\mathbb{H}^{2}$ we can represent any bounded harmonic function $h$ by the formula

$$
h(x)=h_{f}(x)=\int_{\partial \mathbb{H}^{2}} f d \nu_{x}
$$

for some $f \in L^{\infty}\left(\partial \mathbb{H}^{2}\right)$ where $\partial \mathbb{H}^{2}$ is the circle at infinity and $\nu_{x}$ are the harmonic measures. Representing $\mathbb{H}^{2}$ by the unit disk in $\mathbb{C}$, the harmonic measure corresponding to the origin, $\nu_{0}$, is just the unit Lebesgue measure on $S^{1}$. The others are given by

$$
\frac{d \nu_{x}}{d \nu_{0}}(z)=\frac{1-|x|^{2}}{|z-x|^{2}}
$$

The measures $\nu_{x}$ also arise from $\nu_{0}$ by image measures under the transitive isometry group: $\nu_{x}=g_{*} \nu_{0}$ for any $g \in \operatorname{Isom}\left(\mathbb{H}^{2}\right)$ such that $g(0)=x$.

Since $\nu_{x}$ tends to the dirac measure at $z \in \partial \mathbb{H}^{2}$ as $x \rightarrow z$, we obtain

$$
\left\|h_{f}-h_{g}\right\|_{L^{\infty}\left(\mathbb{H}^{2}\right)}=\operatorname{ess~sup}_{x \in X}\left|\int_{\partial \mathbb{H}^{2}} f(z)-g(z) d \nu_{x}(z)\right|=\|f-g\|_{L^{\infty}\left(\partial \mathbb{H}^{2}\right)} .
$$

In other words, we have a maximum principle so that the map $f \mapsto h_{f}$ determines an isometry, with respect to the $L^{\infty}$ norms, between the Banach spaces $H^{\infty}\left(\mathbb{H}^{2}\right)$ of all bounded harmonic functions and $L^{\infty}\left(\partial \mathbb{H}^{2}, \nu_{0}\right)$. Another consequence of the maximum principle is that harmonic functions satisfy the averaging condition. If we identify $\mathbb{H}^{2}$ with any transitive Lie subgroup of $\operatorname{Isom}\left(\mathbb{H}^{2}\right)$, then at each point $x \in \mathbb{H}^{2}$ any harmonic function $h_{f}$ satisfies

$$
h_{f}(x)=\int_{S} h_{f}(x y) d \mu(y)
$$

where $\mu$ is the uniform measure on the unit distance circle $S \subset \mathbb{H}^{2}$ around the identity element with respect to the hyperbolic metric.

$\dagger$ The author was supported in part by an NSF postdoctoral fellowship and DMS-0306594.

$\ddagger$ The author was supported in part by an NSF postdoctoral fellowship. 
We can generalize these concepts greatly to any measure space $X$ with a Markov operator $P$. We say that a function $h$ is $P$-harmonic if $P h=h$. A space $B$ together with a family of mutually absolutely continuous measures $\left\{\nu_{x}\right\}_{x \in X}$ is a Poisson Boundary if the map $f \mapsto h_{f}$ given by the Poisson formula

$$
h_{f}(x):=\int_{B} f(y) d \nu_{x}(y)
$$

is an isometry between $L^{\infty}\left(B,\left\{\nu_{x}\right\}\right)$ and bounded $P$-harmonic functions on $X$. In this event, $\nu_{x}$ is called the harmonic measure at $x$. The Poisson boundary is a purely measurable object and is unique up to measurable isomorphism. As such, even when $X$ is a manifold, the underlying space $B$ could be quite different from that of the topological Martin boundary, which gives a representation space for $\mu$ harmonic functions. The Poisson boundary always exists and has many equivalent descriptions. For instance, it can be identified with the space of ergodic components of the shift map $T$ acting on the space of sample paths of the Markov chain on $X$ associated with the operator $P$. The harmonic measures $\nu_{x}$ are the images under the quotient map of the measures $\mathbb{P}_{x}$ in the path space corresponding to starting the Markov process from state $x \in X$. For other characterizations of the Poisson boundary see Kai03.

Before moving to random walks on groups, we recall the notion of convolution measures. Suppose $(X, \nu)$ is a measure space and $\Gamma$ is any set of $\nu$-measurable transformations of $X$. For any measure $\mu$ on $\Gamma$ we define the convolution of the two measures $\mu \star \nu$ to be the measure on $X$ given by

$$
\mu \star \nu:=\int_{\Gamma} \gamma_{*} \nu d \mu(\gamma)
$$

As a special case of the above constructions, we can define the (right sided) random walk determined by a finite measure $\mu$ on a group $G$ as follows. Let $G^{\mathbb{Z}_{+}}=$ $\prod_{i=1}^{\infty} G$ and denote by $\mathbb{P}$ the measure obtained as the image of $\mu \times \mu \times \cdots$ under the map $\left(x_{1}, x_{2}, x_{3}, \ldots\right) \mapsto\left(e, x_{1}, x_{1} x_{2}, x_{1} x_{2} x_{3}, \ldots\right)$. The conditional measure for $\mathbb{P}$ on the $n$-th coordinate of $G^{\mathbb{Z}_{+}}$is the $n$-fold convolution measure $\mu_{n}=\mu^{\star n}$ on $G$. The measure of all paths starting from an initial distribution $\theta$ is $\theta \star \mathbb{P}\left(G^{\mathbb{Z}_{+}}\right)$. In this context, the natural Markov operator associated to the random walk is $P_{\mu}: L^{\infty}(G, \mu) \rightarrow L^{\infty}(G, \mu)$ defined by

$$
P_{\mu}(f)(g)=\int_{G} f(g h) d \mu(h) .
$$

In this setting we call $P_{\mu}$-harmonic functions simply $\mu$-harmonic. Moreover, for a Poisson boundary $\left(B,\left\{\nu_{g}\right\}_{g \in G}\right)$ it follows that $\nu_{g}=g_{*} \nu_{e}$ so we may simply write the boundary as $(B, \nu)$ where $\nu=\nu_{e}$. It follows from the definition of $\mu$-harmonicity and the Poisson formula (0.1) that for all $f \in L^{\infty}(B, \nu)$ at $e$ we have,

$$
\nu(f)=h_{f}(e)=\int_{G} h_{f}(g) d \mu(g)=\int_{G} g_{*} \nu(f) d \mu(g) .
$$

In short, $\mu \star \nu=\nu$, in which case we also say $\nu$ is $\mu$-stationary. The importance of $\mu$ stationary measures is that the Poisson formula (0.1) yields $\mu$-harmonic functions: for any $x \in G$, 


$$
\begin{aligned}
\int_{G} h_{f}(x g) \mu(g) & =\int_{G} \int_{B} f(z) d(x g)_{*} \nu(z) d \mu(g) \\
& =\int_{B} f(z) d x_{*}\left(\int_{G} g_{*} \nu d \mu(g)\right)(z)=\int_{B} f(z) d x_{*} \nu(z)=h_{f}(x) .
\end{aligned}
$$

A measured $G$-space $\left(B^{\prime}, \nu^{\prime}\right)$ is called a $\mu$-boundary of $G$ if the corresponding Poisson formula, $f \mapsto\left(g \mapsto h_{f}(g):=g_{*} \nu^{\prime}(g)\right)$, defines an isometric embedding from $L^{\infty}\left(B^{\prime}, \nu^{\prime}\right)$ into the space of bounded $\mu$-harmonic functions $H^{\infty}(G, \mu)$. Any $\mu$ boundary arises as a $G$-equivariant measurable quotient $\pi:(B, \nu) \rightarrow\left(B^{\prime}, \nu^{\prime}\right)$ of the Poisson boundary $(B, \nu)$ since the induced lift map $\pi_{*}: L^{\infty}\left(B^{\prime}, \nu^{\prime}\right) \rightarrow L^{\infty}(B, \nu)$ is an isometric embedding. In particular, the Poisson boundary can be characterized as the maximal $\mu$-boundary (see the unpublished survey Kai03). Since the Poisson formula still holds, we also have $\mu \star \nu^{\prime}=\nu^{\prime}$ for any $\mu$-boundary $\left(B^{\prime}, \nu^{\prime}\right)$. On the other hand, it does not follow that any $G$-space with a $\mu$-stationary measure is isomorphic to a $\mu$-boundary. However, if $\nu^{\prime}$ is $\mu$-stationary and all of the Dirac measures $\left\{\delta_{z}\right\}_{z \in B^{\prime}}$ occur in the weak-* closure of $\left\{g \nu^{\prime}\right\}_{g \in \Gamma}$, then as in the case of $\mathbb{H}^{2}$ above, we have $\left\|h_{f}-h_{g}\right\|_{L^{\infty}(G, \mu)}=\|f-g\|_{L^{\infty}\left(B^{\prime}, \nu^{\prime}\right)}$ which implies that $\left(B^{\prime}, \nu^{\prime}\right)$ is a $\mu$-boundary. This last condition will hold in the case that the action of $G$ on $\left(B^{\prime}, \nu^{\prime}\right)$ is $\mu$-proximal in the sense of Furstenberg (see Fur73). This construction can be useful for identifying $\mu$-boundaries as subsets of geometrically defined boundaries for $G$.

For example, consider the case $G=\operatorname{Isom}\left(\mathbb{H}^{2}\right)=S L(2, \mathbb{R})$ with a maximal compact subgroup $K=\operatorname{Stab}(o) \equiv S O(2)$ for a fixed basepoint $o \in \mathbb{H}^{2}$. Let $m_{K}$ denote the (bi-invariant) Haar measure on $K$ which we will think of as a measure on $G$ supported on $K$, and choose an element $g \in G$ such that $d(o, g o)=1$. If $\mu_{0}=m_{K} \star g_{*} m_{K}$, then $\mu_{0}$-harmonic functions on $G$ are right $K$ invariant and their quotients in $G / K=\mathbb{H}^{2}$ are ordinairy harmonic functions. Moreover every $\mu_{0}$ harmonic function is the lift of one on $\mathbb{H}^{2}$. Hence $\left(\partial \mathbb{H}^{2}, \nu_{0}\right)$ is the Poisson boundary of $\left(G, \mu_{0}\right)$.

In fact this same correspondence was established by Furstenberg in Fur63 for any symmetric space $G / K$ where $G$ is semisimple Lie group of noncompact type and $K$ is a maximal compact subgroup. If we again take the bi- $K$ invariant measure $\mu_{0}=m_{K} \star g_{*} m_{K}$, then the Poisson boundary of $\left(G, \mu_{0}\right)$ is the Furstenberg boundary $G / P$ together with the unique $K$-invariant measure $\nu_{0}$. Later, Furstenberg extended this in Fur67 and Fur71 to show that for any lattice $\Gamma<G$, one can build a measure $\mu$ on $\Gamma$ for which $\left(G / P, \nu_{0}\right)$ is the Poisson boundary. Geometric intuition makes it tempting to believe that passing from a Lie Group to a lattice, at least a uniform lattice, should be a simple operation when it comes to garnering asymptotic information of any kind. However, the measure $\mu$ constructed by Furstenberg is quite different from the measure $\mu_{0}$ on $G$. For instance, there are no $K$-invariant measures on the lattice and the measure $\mu$ need not be compactly supported. At least in the rank one case, we will show that there is an infinite dimensional space of measures $\mu$ on $\Gamma$ for which $\left(G / P, \nu_{0}\right)$ is a Poisson boundary.

Furstenberg proved this result for $\Gamma$ in two steps. First he constructed a $\mu$ for which $\nu_{0}$ was $\mu$-stationary. By the argument mentioned above he concluded that this was a $\mu$-boundary. Next he showed that any $\mu$-boundary which has finite first 
moment is the Poisson boundary. Here finite first moment means,

$$
\sum_{\gamma \in \Gamma} \mu(\gamma) d(e, \gamma)<\infty
$$

He used this geometric characterization of the Poisson boundary to distinguish envelopes of discrete groups. Namely, a discrete group $G$ cannot be a lattice in $S L(n, \mathbb{R})$ for two different values of $n$.

Kaimanovich and Vershik in KV83 gave sufficient and necessary conditions for the Poisson boundary of a random walk on a locally compact group to be trivial. In Kai00, Kaimanovich generalized this to a criterion to decide when a certain geometric boundary for a group together with a family of exit measures could be a Poisson boundary for a given random walk on the group. For $(B, \nu)$ to be a Poisson boundary for $(G, \mu)$ they showed that in addition to stationarity, $\mu \star \nu=\nu$, it is sufficient for $\mu$ to have both finite first log-moment and finite entropy:

$$
\sum_{\gamma \in \Gamma} \mu(\gamma) \log d(e, \gamma)<\infty \quad \text { and } \quad \mathrm{h}(\mu):=-\sum_{\gamma \in \Gamma} \mu(\gamma) \log \mu(\gamma)<\infty .
$$

The goal of the present paper is to generalize Furstenberg's results to Gromov hyperbolic groups and to a general class of boundary measures. By so doing, we can partially answer a converse question to that answered by Kaimanovich and Vershik's results stated above. Namely, starting with a measure $\nu$ on $\partial G$ can we find a measure $\mu$ on $G$ such that $(\partial G, \nu)$ is the Poisson boundary of $G$ ? In fact, not every measure $\nu$ can arise as a Poisson boundary, and there are examples of measures $\nu$ which do arise but $f \nu$ does not for certain positive measurable functions $f$ (see Remark 5.3). Nevertheless, we shall give an affirmative answer for any measure Lipschitz equivalent to a Patterson-Sullivan measure on a CAT $(-1)$ group. If one asks the same question for $\mu$-boundaries instead, then we show existence for continuous measures in this class on a large family of groups which includes the Gromov hyperbolic groups.

In a second paper, we will broaden some of these results to multiple measure classes within the family of Gibbs streams.

To achieve the stated goal we will extend the approach of Furstenberg's original work (Fur63] ) to our wider context. We restrict our attention here to spaces which are negatively curved in a general sense. We hold out the hope that in the future some of these techniques should also be able to address similar problems for certain nonamenable nonpositively curved groups.

Consider a locally compact $\delta$-hyperbolic metric space $(X, d)$, and let $\Gamma$ be an arbitrary group of isometries of $X$. We will assume $(X, d, \Gamma)$ satisfies two mild conditions which we call "Gromov product bounded" and "uniformly quasigeodesic" which hold whenever $X$ is a complete geodesic space. The first, guarantees that the metric is well behaved near infinity and the second guarantees that points are sufficiently well distributed in $X$. Since $\Gamma$ acts by isometries on $\partial X$, we can consider its ideal limit set $\Lambda \subset \partial X$ and radial (or conical) limit set $\Lambda_{r} \subset \Lambda$ (see Section 1). To avoid dealing with uninteresting cases, we assume $\Gamma$ is nonelementary. This means that $\Lambda$ has at least 3 points in it. Note that $\Gamma$ itself need not be a hyperbolic group. In fact, many relatively hyperbolic convergence groups (in the sense of Bowditch $[\mathrm{Bow}$ ) will satisfy the hypotheses of our first theorem below. For instance, if $X=\mathbb{H}^{3}$ and $\Gamma$ is a geometrically finite discrete group of isometries then the 
complement of $\Lambda_{r}$ in $\Lambda$ is a countable dense set of points, and $\Lambda$ may have topological codimension 1 or 2 in $\partial X$.

The metric structure $(X, d)$ induces a natural 1-parameter family of metrics on $\partial X$ parameterized by $\epsilon$. If $\Gamma$ is discrete and acts properly discontinuously, then the metric corresponding to $\epsilon$ gives $\Lambda$ Hausdorff dimension $\delta(\Gamma) / \epsilon$, where $\delta(\Gamma)$ is the critical exponent for the action of $\Gamma$. The corresponding Hausdorff measure is in the same measure class as the Patterson-Sullivan measures. If the Gromov product extends continuously to $\partial X$, then these measures are examples of continuous $\alpha$ quasiconformal densities for $\alpha=\delta(\Gamma)$ (see Section 1 for all definitions and details).

Theorem 0.1 (Stationarity). Let $X$ be a Gromov product bounded, uniformly quasigeodesic, $\delta$-hyperbolic space and choose a nonelementary $\Gamma<\operatorname{Isom}(X)$ with limit set $\Lambda \subset \partial X$. Let $\nu$ be a continuous $\alpha$-quasiconformal measure on $\Lambda \subset \partial X$ for any $\alpha>0$. Suppose $\nu^{\prime}$ is an equivalent measure whose Radon-Nikodym derivative $\frac{d \nu^{\prime}}{d \nu}$ is a uniformly positive lower semicontinuous function. Assume $\Gamma$ posesses a radial limit set $\Lambda_{r}$ of full $\nu$-measure in $\Lambda$. If $(X, \Gamma)$ is quasiconvex cobounded or else $\nu$ belongs to a bounded quasiconformal density, then there exists a (nontrivial) measure $\mu$ on $\Gamma$ such that $\mu \star \nu=\nu^{\prime}$.

Remark 0.2 . The assumption that $X$ is uniformly quasiconvex and that $\Gamma$ is quasiconvex cobounded in the case that $\nu$ does not arise from a density are only used to establish a decay condition for $\nu$ (3.1) which akin to a weakened form of upper Ahlfors regularity. In particular, these conditions can be dropped if this regularity can be established by some other means. Note that the uniformly quasiconvex assumption is much weaker than being geodesic.

Coornaert showed in Coo93 that when $X$ is geodesic, $\alpha$-quasiconformal densities only exist for $\alpha \geq \delta(\Gamma)$. When $X$ is not geodesic this follows from our version of Sullivan's Shadow Lemma (1.26). The main significance of the above theorem is the following.

Corollary 0.3. If $\Gamma, X$ and $\nu$ are as in the above theorem, then $(\Lambda, \nu)$ is a $\mu$ boundary of $\Gamma$.

Unfortunately, even in the case of negatively curved manifolds, it does not follow that $(\Lambda, \nu)$ is a Poisson boundary for $\mu$ despite the fact that $\nu$ is supported on all of $\Lambda$. The problem is twofold: two divergent sequences may actually asymptotically represent the same $\mu$ walk, and two asymptotically metrically convergent sequences may asymptotically represent different $\mu$ walks. To guarantee the maximality of the above boundary we need to connect the large scale behavior of $\mu$ to the large scale behavior of the metric. Kaimanovich Kai00 has formulated very general criterion for establishing maximality. When $X$ is a $\mathrm{CAT}(-\kappa)$ space, we were able to establish these criteria in certain cases resulting in our second main result.

Theorem 0.4 (Poisson Boundary). Suppose that in addition to the hypotheses of Corollary 0.3 we assume $X$ is a CAT $(-\kappa)$ space, $\Gamma$ is locally compact and that a uniform neighborhood of a $\Gamma$ orbit contains the convex hull of $\Lambda$. If $\nu$ is a Lipschitz $\alpha$-quasiconformal measure, then there is a measure $\mu$ on $\Gamma$ such that $(\Lambda, \nu)$ is a Poisson boundary for $(\Gamma, \mu)$.

The most important examples to which we apply this theorem is given by the following result which we prove in Section 8 
Corollary 0.5. If $(X, \Gamma)$ is as in the above theorem and $\nu$ is bounded Lipschitz equivalent to either the Hausdorff measure of a Busemann metric or a PattersonSullivan measure, then there is a measure $\mu$ on $\Gamma$ such that $(\Lambda, \nu)$ is the Poisson boundary for $(\Gamma, \mu)$.

Remark 0.6. In each of the results above, we find solution measures $\mu$ which are an infinite sum of atomic measures. However in Corollary 8.5 we show that, in each case, the family of stationizing measures $\mu$ is infinite dimensional and has members in any Borel measure class supported on all of $\Gamma$. Unfortunately, we were unable to determine whether a symmetric example always exists; i.e. one with $\mu(\gamma)=\mu\left(\gamma^{-1}\right)$ for all $\gamma \in \Gamma$.

We shall prove Theorem 0.4 by showing that both the finite entropy and the finite first log-moment condition are satisfied by a carefully constructed measure given by Theorem 0.1 The results of Kaimanovich mentioned above will finish the proof. Since we do not assume that $\Gamma$ acts cocompactly, there is a strong constraint on the log-moment property, but it will be satisfied whenever the limit set contains a conical limit set of full measure. In fact, we will give criteria in terms of the limit set, for when the usual first moment is finite. This implies some properties of the random walk.

Before concluding the introduction, we mention an explicit application in the following example; a setting which has seen considerable recent interest.

Example 0.7 (Fuchsian buildings). Throughout this example we refer to BP00 for details and proofs of stated facts. Let $R$ be a right angled regular $r$-gon in the hyperbolic plane $\mathbb{H}^{2}$. Given an $r$-tuple of integers $\left(q_{1}, \ldots, q_{r}\right)$ with $q_{i} \geq 2$ we assign the cyclic group of order $q_{i}+1$ to the $i$-th edge and the trivial group to the face of $R$. This gives an "orbihedron" structure to $R$ which is developable in the sense of Hae91. Its universal developing cover $\Delta$ is a two dimensional cell complex called a right-angle Fuchsian building. Moreover, $\Delta$ with the induced path metric is a CAT(-1) space. Let $\Gamma \leqslant I \operatorname{som}(\Delta)$ be the fundamental group of the orbihedron $R$, so that $\Gamma \backslash \Delta=R$. Since $R$ is a compact orbihedron, the limit set and conical limit set coincide, so we may apply Corollary 0.5] In particular, for a Patterson-Sullivan measure $\nu_{p}$ on the boundary $\partial \Delta$ we can find a probability measure $\mu \in P(\Gamma)$ such that $\left(\partial \Delta, \nu_{p}\right)$ is the Poisson boundary for $\mu$. The flexibility of our approach allows us to handle other measures as well. Let $G(\Gamma)$ be the dual graph to the 1-skeleton of $\Delta$. If we adjust the weight of edges by requiring the length of each edge that crosses an edge associated to $q_{i}$ to be $\log \left(q_{i}\right)$, then we obtain a $\delta$-hyperbolic space for some $\delta>0$. Moreover, the Patterson-Sullivan measures for this metric graph satisfy our conditions in Theorem 0.1 The associated Busemann metric based at each chamber $p$ is called combinatorial metric $\delta_{p}$. Denote the Hausdorff measure of $\delta_{p}$ by $\mathcal{H}_{p}^{\text {comb }}$. The most important thing is that the Hausdorff measures form a conformal density $\frac{d\left(\gamma^{*} \mathcal{H}_{p}^{c o m b}\right)}{d \mathcal{H}_{p}^{\text {comb }}}(z)=e^{(\tau+1) N_{z}\left(p, \gamma^{-1} p\right)}$ where $\tau$ is the critical exponent of $\Gamma$ with respect to $\delta_{p}$ and $N_{z}$ is the associated Busemann function. Moreoever, this is a locally constant, hence continuous, function on the boundary $\partial \Delta$ outside of a set of measure 0 (this set is the complement of the so called tree-wall ends) ${ }^{1}$. So we can apply Corollary $\left[0.3\right.$ to conclude that there exists a measure $\mu^{\prime} \in P(\Gamma)$ such

\footnotetext{
${ }^{1}$ Personal communication with Marc Bourdon
} 
that $\mu^{\prime} \star \mathcal{H}_{p}^{c o m b}=\mathcal{H}_{p}^{c o m b}$ and $\left(\partial \Delta, \mathcal{H}_{p}^{c o m b}\right)$ is a $\mu$-boundary. Since the PattersonSullivan measures have the same Radon-Nikodym derivatives, the ergodicity of the action of $\Gamma$ on $\Lambda$ implies that the Patterson-Sullivan measures of the combinatorial metric coincide up to a constant multiple with the Hausdorff measure with respect to the same base chamber (see Proposition 1.29).

We conclude with a brief outline of the paper. In Section 1 we introduce the basic tools used in working with nongeodesic Gromov hyperbolic spaces, and we generalize some well known estimates to this setting for later use. Section 2 sets up the presentation of Patterson-Sullivan theory in this context. Sections 3 and 4 present the notation and background for the conditions which will be needed in order to guarantee that a family of functions can form a positive basis. In Section [5] we present a general theorem of independent interest which establishes when lower semicontinuous functions can be approximated by positive sums of basis functions. In Section 6 this theorem is extended to give conditions for which the functional approximations can be done with finite first moment or log-moment. These theorems are applied to the case of Patterson-Sullivan measures in Sections 7 and 8 where the two main theorems are also proved. Finally we demonstrate the theorems in the simple example of a free group in Section 9

\section{ACKNOWLEDGEMENTS}

Both authors would like to thank Alex Eskin, Alex Furman and Vadim Kaimanovich for helpful communications, and Yehuda Shalom and Marc Bourdon for pointing out the application in Example 0.7 The second author would also like to thank Gregory Margulis and Carlos Kenig for insightful discussions.

\section{BACKGROUND ON $\delta$-HYPERBOLIC SPACES}

We first recall the definition of a geodesic Gromov $\delta$-hyperbolic metric space. After doing so, we recall the general definition which may be found in [BH99]. The remainder of the section is devoted to reconstructing the basic facts about such spaces which we will need later.

\subsection{Geodesic $\delta$-hyperbolic spaces.}

Definition 1.1. We say that a geodesic metric space $(X, d)$ is $\delta$-hyperbolic if for every geodesic triangle $\Delta \subset X$, each side of $\Delta$ has Hausdorff distance at most $\delta$ to the union of the other two sides. Equivalently, each side is contained in the uniform $\delta$ neighborhood of the other two sides.

1.2. General definition. First we define one of the basic quantities used in our asymptotic analysis.

Definition 1.2. Let $(X, d)$ be a metric space and $x \in X$. The Gromov product of $y, z \in X$ with respect to $x$ is defined to be

$$
(y \cdot z)_{x}=\frac{1}{2}(d(x, y)+d(x, z)-d(y, z)) .
$$

It is easy to observe that $(y \cdot z)_{x} \leq \min \{d(x, y), d(x, z)\}$. Now we present a definition of $\delta$-hyperbolic space without resorting to geodesics. 
Definition 1.3. Let $\delta \geq 0$. A metric space $(X, d)$ is said to be $\delta$-hyperbolic (or Gromov hyperbolic) if

$$
(x \cdot y)_{w} \geq \min \left\{(x \cdot z)_{w},(y \cdot z)_{w}\right\}-\delta,
$$

for all $w, x, y, z \in X$.

For geodesic spaces, we could allow $w$ in the second definition to vary along one side of the triangle $\Delta(x, y, z)$. A simple application of the triangle inequality then shows that a $\delta$-hyperbolic space in the second sense is $2 \delta$-hyperbolic in the first sense. The converse is proved in Chapter III.H of [BH99].

Definitions 1.4. We say a sequence $\left(x_{i}\right)$ of points in $X$ converges at infinity if $\left(x_{i} \cdot x_{j}\right)_{p} \rightarrow \infty$ as $i, j \rightarrow \infty$. Two sequences converging at infinity, $\left(x_{i}\right)$ and $\left(y_{i}\right)$, are equivalent if $\left(x_{i} \cdot y_{j}\right)_{p} \rightarrow \infty$ as $i, j \rightarrow \infty$. The space of all equivalence classes of sequences converging to infinity is denoted by $\partial X$, and is called the boundary of $X$. For a sequence $\left(x_{i}\right)$ converging at infinity and an equivalence class $x \in \partial X$, we write $x=\lim _{i \rightarrow \infty} x_{i}$ if $\left(x_{i}\right) \in x$.

Note that if $X$ is geodesic, then there is a natural bijection from this boundary to the geodesic boundary. Now we can extend the definition of Gromov product to $\partial X$.

Definition 1.5. Let $(X, d)$ be a $\delta$-hyperbolic space with base point $p \in X$. Let $\bar{X}=X \cup \partial X$. We extend the Gromov product to $x, y \in \bar{X}$ by

$$
(x \cdot y)_{p}=\sup \liminf _{i, j \rightarrow \infty}\left(x_{i} \cdot y_{j}\right)_{p},
$$

where the supremum is taken over all sequences $\left(x_{i}\right)$ and $\left(y_{j}\right)$ in $X$ such that $x=$ $\lim _{i \rightarrow \infty} x_{i}$ and $y=\lim _{j \rightarrow \infty} y_{j}$. (As a consequence, $(x \cdot y)_{p}=\sup \limsup _{i, j \rightarrow \infty}\left(x_{i} \cdot y_{j}\right)_{p}$.)

Since the metric $d$ is by definition continuous, the extended Gromov product and the original Gromov product agree on $X \times X$. The extended product allows us to define a topology on $\bar{X}$. Namely, a set is closed if and only if it contains all of its limit points.

Proposition 1.6. (BH99) Let $X$ be a $\delta$-hyperbolic space and fix $p \in X$.

(1) The extended product $(\cdot)_{p}$ is continuous on $X \times X$, but not necessarily on $\bar{X} \times \bar{X}$.

(2) In the definition of $(x \cdot y)_{p}$, if we have $x \in X$ (resp. $\left.y \in X\right)$, then we may always take the respective sequence to be the constant value $x_{i}=x$ (resp. $\left.y_{j}=y\right)$.

(3) For all $x, y \in \bar{X}$ there exist sequences $\left(x_{n}\right)$ and $\left(y_{n}\right)$ such that $x=\lim _{n \rightarrow \infty} x_{n}$ and $y=\lim _{n \rightarrow \infty} y_{n}$ and $(x \cdot y)_{p}=\lim _{n \rightarrow \infty}\left(x_{n} \cdot y_{n}\right)_{p}$.

(4) For all $x, y, z \in \bar{X}$ by taking limits we still have

$$
(x \cdot y)_{p} \geq \min \left\{(x \cdot z)_{p},(y, \cdot z)_{p}\right\}-2 \delta .
$$

(5) For all $x, y \in \partial X$ and all sequences $\left(x_{i}^{\prime}\right)$ and $\left(y_{j}^{\prime}\right)$ in $X$ with $x=\lim _{i \rightarrow \infty} x_{i}^{\prime}$ and $y=\lim _{j \rightarrow \infty} y_{i}^{\prime}$,

$$
(x \cdot y)_{p}-2 \delta \leq \liminf _{i, j \rightarrow \infty}\left(x_{i} \cdot y_{j}\right)_{p} \leq(x \cdot y)_{p} .
$$


Recall that a $(\lambda, C)$-quasi-isometric embedding between two metric spaces $\left(X, d_{X}\right)$ and $\left(Y, d_{Y}\right)$ is a map $f: X \rightarrow Y$ such that for all $x, y \in X$,

$$
\frac{1}{\lambda} d_{X}(x, y)-C \leq d_{Y}(f x, f y) \leq \lambda d_{X}(x, y)+C .
$$

A $C$-quasigeodesic in $X$ is the image of a $(1, C)$-quasi-isometric embedding of $\mathbb{R}$ into $X$.

Definition 1.7. We say that a metric space $X$ is $(C$-) uniformly quasigeodesic if there is a $C \geq 0$ such that for any two distinct points in $X$ there is a $C$-quasigeodesic joining them. In particular, a 0 -uniformly quasigeodesic space is geodesic.

Definition 1.8. Let $(X, d)$ be a $\delta$-hyperbolic space with base point $p \in X$. Fix $\epsilon>0$. We consider the following measure of separation of the points in $\bar{X}$

$$
d_{p}^{\epsilon}(x, y)=e^{-\epsilon(x \cdot y)_{p}},
$$

for $x, y \in \bar{X}$. Denote $d_{p}^{1}=d_{p}$.

1.3. Metrics on $\partial X$. Now we recall the existence of a compatible metric on $\partial X$. (See BH99 or GdlH90 for the nongeodesic case.)

Proposition 1.9. Gro87 Let $(X, d)$ be a $\delta$-hyperbolic space. If $0<\epsilon \leq \frac{\log 2}{4 \delta}$, then there exists a metric $\delta_{p}^{\epsilon}$ on $\partial X$ so that

$$
\left(3-2 e^{2 \delta \epsilon}\right) d_{p}^{\epsilon}(z, y) \leq \delta_{p}^{\epsilon}(z, y) \leq d_{p}^{\epsilon}(z, y),
$$

for all $z, y \in \partial X$

Since the Gromov product is nonnegative, the above proposition implies $\operatorname{diam}(\partial X) \leq$ 1 in the metric $\delta_{p}^{\epsilon}$ for any $p \in X$ and $0<\epsilon \leq \frac{\log 2}{4 \delta}$. Moreover, we have

Proposition 1.10 (6.2 of [BS00]). With respect to $\delta_{p}^{\epsilon}$, the boundary $\partial X$ is complete.

Note that $\partial X$ need not be compact. For instance, the boundary of $\mathbb{H}^{\infty}$ is the Hilbert sphere. By taking a tree whose level $n$ leaves form a $1 / n$ net in the $n$ dimensional sphere, one obtains a locally compact example with the same boundary. On the other hand, if all of the closed balls in $X$ are compact, then we say $X$ is proper. This condition is much stronger, and we omit the (straightforward) proof of the following.

Proposition 1.11. If $X$ is a proper $\delta$-hyperbolic space, then $\partial X$ is compact and $\operatorname{Isom}(X)$ is locally compact.

With some difficulty we avoid using this assumption on $X$. In fact, we don't even assume $\Gamma$ is locally compact until Theorem 0.4 and its corollaries. The point is to allow groups in the first theorem which come from infinite dimensional constructions.

Recall that a $C$-quasimetric is a function $d: X \times X \rightarrow \mathbb{R}$ satisfying all of the properties of a metric except for the triangle inequality which is substituted by the condition that $d(x, y) \leq C(d(x, z)+d(z, y))$ for all $x, y, z \in X$ and some $C \geq 1$. Proposition 1.9 implies that $d_{p}^{\epsilon}(z, y)$ is a $\frac{1}{\left(3-2 e^{2 \delta \epsilon}\right)}$-quasimetric which can be used in most computations instead of the more complicated metric. However, the $s$ power of a $C$-quasimetric is a $C 2^{s-1}$-quasimetric, so for any $s>0$ and any $0<\epsilon<\frac{\log 2}{4 \delta}$, $d_{p}^{s}$ is a $\frac{2^{\frac{s}{\epsilon}-1}}{3-2 e^{2 \delta \epsilon}}$-quasimetric. Whenever $\delta>\frac{(4+3 \sqrt{2}) \log (2)}{4 s}$, the choice $\epsilon=\frac{\log 2}{4 \delta}$ is optimal in the valid interval giving that $d_{p}^{s}$ is a $\left(\frac{3}{2}+\sqrt{2}\right) e^{4 \delta s}$-quasimetric. 
Lemma 1.12. Let $(X, d)$ be a $\delta$-hyperbolic space. Fix two points $p, q \in X$. Then for all $z, y \in \partial X$ and $C>0$ such that

$$
d_{p}(z, y) \leq e^{C} e^{-d(p, q)} \leq e^{C} \min \left\{e^{-(z \cdot q)_{p}}, e^{-(y \cdot q)_{p}}\right\},
$$

we have $\left|(z \cdot q)_{p}-(y \cdot q)_{p}\right| \leq 2 \delta+C .\left(\right.$ Recall that $\max \left\{(y \cdot q)_{p},(z \cdot q)_{p}\right\} \leq d(p, q)$.)

Proof. Without loss of generality assume that $(y \cdot q)_{p} \geq(z \cdot q)_{p}$. Since $d_{p}(z, y)=$ $e^{-(z \cdot y)_{p}}$, we have

$$
(z \cdot y)_{p} \geq d(p, q)-C \geq \max \left\{(z \cdot q)_{p},(y \cdot q)_{p}\right\}-C=(y \cdot q)_{p}-C .
$$

By Item 4in Proposition 1.6 we have

$$
\begin{aligned}
(z \cdot q)_{p} & \geq \min \left\{(y \cdot q)_{p},(z \cdot y)_{p}\right\}-2 \delta=\min \left\{(y \cdot q)_{p},(y \cdot q)_{p}-C\right\}-2 \delta \\
& =(y \cdot q)_{p}-2 \delta C .
\end{aligned}
$$

This proves the lemma.

Now we present some estimates we shall need later for how $d_{p}(x, y)$ varies on $\bar{X}$.

Lemma 1.13. Let $(X, d)$ be a $\delta$-hyperbolic space and $p, q \in X$. Let $U_{p}(q)=$ $\sup _{z \in \partial X}\left\{(z \cdot q)_{p}\right\}$. Let $z_{p, q}$ be any point in $\partial X$ such that $\left(z_{p, q} \cdot q\right)_{p} \geq U_{p}(q)-\delta$.

a) For all $y \in \partial X$, we have

$$
e^{(y \cdot q)_{p}} \leq \frac{e^{3 \delta}}{d_{p}\left(z_{p, q}, y\right)}
$$

b) If $d_{p}\left(z_{p, q}, y\right) \geq e^{-U_{p}(q)}$, then

$$
e^{(y \cdot q)_{p}} \geq \frac{e^{-3 \delta}}{d_{p}\left(z_{p, q}, y\right)}
$$

Proof. For part a), recall that

$$
\left(z_{p, q} \cdot y\right)_{p} \geq \min \left\{\left(z_{p, q} \cdot q\right)_{p},(y \cdot q)_{p}\right\}-2 \delta .
$$

Since $\left(z_{p, q} \cdot q\right)_{p} \geq U_{p}(q)-\delta$ and $U_{p}(q) \geq(y \cdot q)_{p}$, we obtain that

$$
\left(z_{p, q} \cdot y\right)_{p} \geq \min \left\{(y \cdot q)_{p}-\delta,(y \cdot q)_{p}\right\}-2 \delta=(y \cdot q)_{p}-3 \delta .
$$

For b), the condition $d_{p}\left(z_{p, q}, y\right) \geq e^{-U_{p}(q)}$ implies that $\left(z_{p, q} \cdot y\right)_{p} \leq U_{p}(q)$. Since $\left(z_{p, q} \cdot q\right)_{p} \geq U_{p}(q)-\delta$, we have

$$
\begin{aligned}
(y \cdot q)_{p} & \geq \min \left\{\left(z_{p, q} \cdot q\right)_{p},\left(z_{p, q} \cdot y\right)_{p}\right\}-2 \delta \geq \\
& \geq \min \left\{\left(z_{p, q} \cdot y\right)_{p}-\delta,\left(z_{p, q} \cdot y\right)_{p}\right\}-2 \delta=\left(z_{p, q} \cdot y\right)_{p}-3 \delta .
\end{aligned}
$$

This proves the lemma. 
1.4. Isometric actions on $\bar{X}$. Assume that a group $\Gamma$ acts by isometries on $X$. Fix $p \in X$.

One easily observes that $(\gamma x \cdot \gamma y)_{\gamma p}=(x \cdot y)_{p}$ for all $x, y \in X$ and $\gamma \in \Gamma$. The same is true for all $x, y \in \bar{X}$. For if $\left(x_{n}\right)$ and $\left(y_{n}\right)$ are two sequences such that

$$
(x \cdot y)_{p}=\lim _{n \rightarrow \infty}\left(x_{n}, y_{n}\right)_{p}
$$

then

$$
\lim _{n \rightarrow \infty}\left(\gamma x_{n}, \gamma y_{n}\right)_{\gamma p}=(\gamma x, \gamma y)_{\gamma p}
$$

For a similar proof of the following in the manifold case, see Yue96.

Lemma 1.14. For all $x, y \in \partial X$ and all $p, q \in X$ we have

$$
e^{-d(p, q)-2 \delta} e^{\left((x \cdot q)_{p}+(y \cdot q)_{p}\right)} \leq \frac{d_{q}(x, y)}{d_{p}(x, y)} \leq e^{-d(p, q)+2 \delta} e^{\left((x \cdot q)_{p}+(y \cdot q)_{p}\right)} .
$$

Proof. Let $x, y \in \partial X$. Assume that $\left(x_{n}\right)$ and $\left(y_{n}\right)$ are two sequences in $X$ such

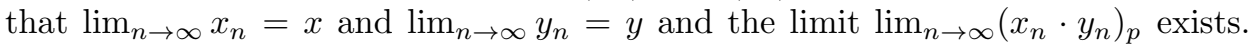
By item 3 in Proposition [1.6 the sequence $\left(x_{n}\right)$ and $\left(y_{n}\right)$ may be chosen so that $\lim _{n \rightarrow \infty}\left(x_{n} \cdot q\right)_{p}=(x \cdot q)_{p}$ and $\lim _{n \rightarrow \infty}\left(y_{n} \cdot q\right)_{p}=(y \cdot q)_{p}$.

By hyperbolicity, we have

$$
(x \cdot y)_{p}-2 \delta \leq \lim _{n \rightarrow \infty}\left(x_{n} \cdot y_{n}\right)_{p} \leq(x \cdot y)_{p},
$$

and

$$
(x \cdot y)_{q}-2 \delta \leq \liminf _{n \rightarrow \infty}\left(x_{n} \cdot y_{n}\right)_{q} \leq(x \cdot y)_{q}
$$

Therefore

$\liminf _{n \rightarrow \infty}\left(\left(x_{n} \cdot y_{n}\right)_{q}-\left(x_{n} \cdot y_{n}\right)_{p}\right)-2 \delta \leq(x \cdot y)_{p}-(\gamma x \cdot \gamma y)_{p} \leq \liminf _{n \rightarrow \infty}\left(\left(x_{n} \cdot y_{n}\right)_{q}-\left(x_{n} \cdot y_{n}\right)_{p}\right)+2 \delta$.

Now observe that

$$
\left(x_{n} \cdot y_{n}\right)_{q}-\left(x_{n} \cdot y_{n}\right)_{p}=-\left(x_{n} \cdot q\right)_{p}-\left(y_{n} \cdot q\right)_{p}+d(p, q) .
$$

So we obtain,

$$
-(x \cdot q)_{p}-(y \cdot q)_{p}+d(p, q)-2 \delta \leq(x \cdot y)_{q}-(x \cdot y)_{p} \leq-(x \cdot q)_{p}-(y \cdot q)_{p}+d(p, q)+2 \delta .
$$

Therefore,

$$
\frac{d_{q}(x, y)}{d_{p}(x, y)}=\frac{e^{-(x \cdot y)_{q}}}{e^{-(x \cdot y)_{p}}}=e^{-\left((x \cdot y)_{q}+(x \cdot y)_{p}\right)} \leq e^{2 \delta} e^{-d(p, q)} e^{\left((x \cdot q)_{p}+(y \cdot q)_{p}\right)},
$$

and

$$
\frac{d_{q}(x, y)}{d_{p}(x, y)} \geq e^{-2 \delta} e^{-d(p, q)} e^{\left((x \cdot q)_{p}+(y \cdot q)_{p}\right)}
$$

Corollary 1.15. For all $x, y \in \partial X$ and any $p, q \in X$ we have

$$
e^{(d(p, q)-2 \delta)} e^{-\left((x \cdot p)_{q}+(y \cdot p)_{q}\right)} \leq \frac{d_{q}(x, y)}{d_{p}(x, y)} \leq e^{(d(p, q)+2 \delta)} e^{\left((x \cdot p)_{q}+(y \cdot p)_{q}\right)} .
$$


Proof. Let $\left\{x_{n}\right\}$ be a sequence in $X$ such that $\lim _{n \rightarrow \infty}\left(x_{n} \cdot q\right)_{p}=(x \cdot q)_{p}$. Since $\left(x_{n} \cdot p\right)_{q}=d(q, p)-\left(x_{n} \cdot q\right)_{p}$ and $\liminf _{n \rightarrow \infty}\left(x_{n} \cdot p\right)_{q} \leq(x \cdot p)_{q}$, we have

$$
d(p, q)-(x \cdot q)_{p} \leq(x \cdot p)_{q} .
$$

Similarly, we have $d(p, q)-(x \cdot p)_{q} \leq(x \cdot q)_{p}$. So $d(p, q)-(x \cdot q)_{p}=(x \cdot p)_{q}$. This proves the corollary.

Before concluding this section, we will introduce a few more definitions and prove the Shadow Lemma for $\delta$-hyperbolic spaces.

Definitions 1.16. For fixed $x, p \in X$, let $\Lambda=\Lambda(\Gamma)$ denote the subset of $\partial X$ consisting of all asymptotic equivalence classes, with respect to $(\cdot)_{p}$, of sequences of the form $\left(\gamma_{i} x\right)$ for $\gamma_{i} \in \Gamma$. The set $\Lambda$ is called the limit set of $\Gamma$ and is sometimes denoted as $\partial \Gamma$.

The radial (or conical) limit set of $\Gamma$, denoted by $\Lambda_{r}$, is the subset of $\Lambda$ such that $z \in \Lambda_{r}$ if and only if there is a constant $C>0$ and a sequence $g_{i} \in \Gamma$ with $\left(g_{i} x\right)$ converging to $z$ such that $d\left(g_{i} x, p\right)-\left(g_{i} x \cdot z\right)_{p} \leq C$. Note that by Proposition [1.6] this is equivalent to $(p \cdot z)_{g_{i} x} \leq C^{\prime}$ for some constant $C-2 \delta<C^{\prime}<C+2 \delta$. In the case of a geodesic space, we may express this condition by saying that the orbit subsequence $\left(g_{i} x\right)$ must stay within distance $C$ of the geodesic between $p$ and $z$.

It is easy to see that these definitions do not depend on the choice of $p$ or $x$, so long as we are free to change the constant $C$.

Definitions 1.17. For a geodesic space $X$, the geodesic hull (sometimes called the Gromov envelope) of $\Lambda, \mathrm{GH}(\Lambda)$ is the union of all geodesics in $X$ with both endpoints in $\Lambda$. The convex hull of $\Lambda, \mathrm{CH}(\Lambda)$, is the smallest subset of $X$ containing $\mathrm{GH}(\Lambda)$ with the property that every geodesic segment between any pair of points $p, q \in \mathrm{CH}(\Lambda)$ also lies in $\mathrm{CH}(\Lambda)$. If $X$ is $C$-uniformly quasigeodesic, then we let $\mathrm{GH}(\Lambda)$ be the union of all $C$-quasigeodesics with both endpoints in $\Lambda$. Here we assume this constant $C$ has been chosen once and for all.

We say that $X$ is quasiconvex cocompact (resp. quasiconvex cobounded) with respect to the action of $\Gamma$ if $\mathrm{GH}(\Lambda) / \Gamma$ is compact (resp. $\mathrm{GH}(\Lambda)$ lies in the uniform neighborhood of some $\Gamma$-orbit). Similarly, the action of $\Gamma$ on $X$ is convex cocompact if $\mathrm{CH}(\Lambda) / \Gamma$ is compact.

It is important to note that $\mathrm{CH}(\Lambda)$ is strictly larger than $\mathrm{GH}(\Lambda)$ even for (most) actions of surface groups on $\mathbb{H}^{3}$. Nevertheless, for CAT(-1) spaces (see Section 2), the notion of quasiconvex cocompactness is equivalent to convex cocompactness. The quasiconvex cocompact actions are the simplest families of examples where $\Lambda_{r}$ and $\Lambda$ coincide.

Definitions 1.18. We say that a $\delta$-hyperbolic space $X$ is upper (resp. lower) Gromov product bounded from above (resp. below) if there exists a $p \in X$ and a constant $C$ such that

$$
\sup _{z \in \partial X}(z \cdot q)_{p} \geq d(p, q)-C, \quad\left(\inf _{z \in \partial X}(z \cdot q)_{p} \leq C,\right)
$$

for all $q \in X$. The space $X$ is Gromov product bounded if it is both upper and lower Gromov product bounded. The $\delta$-hyperbolic space $X$ is weakly Gromov product bounded if there exists a constant $C$ and $p \in X$ such that

$$
\inf _{z \in \partial X}(z \cdot q)_{p}+\sup _{z \in \partial X}(z \cdot q)_{p}-d(p, q) \leq C,
$$


for all $q \in X$. Lastly we say $X$ is (weakly,upper,lower) Gromov bounded with respect to $\Gamma$ if we only require the corresponding condition to hold for $q \in \Gamma \cdot p$. A straightforward application of the triangle inequality shows that if any of these conditions holds for one $p \in X$, then it also holds for every $p \in X$ for an appropriate choice of $C$.

If $\Gamma$ acts cocompactly, then any $p$ would do, and we could choose $C$ independently of $p$. Also, if $X$ is complete and geodesic, then we can set $C=0$.

Being Gromov product bounded from above (respectively below) morally means that for each $q \in X$ there is a point $z \in \partial X$ which assumes the role of the forward (backward) endpoint of a geodesic through $p$ and $q$, even though no such geodesic need exist. In particular, if for every $q \in X, p$ and $q$ are always connected by a discrete $C$-quasigeodesic, then $X$ is Gromov product bounded.

1.5. Quasiconformal Measures on $\Lambda$. We will need to consider certain classes of measures which transform nicely under the $\Gamma$ action.

Definitions 1.19. Let $\nu$ be a Borel measure on $\partial X$. We say that $\nu$ is upper (resp. lower) $\alpha$-quasiconformal for $\Gamma$ if for some $p \in X$,

$\frac{d \gamma_{\star} \nu}{d \nu}(z) \leq C e^{-\alpha d\left(p, \gamma^{-1} p\right)} e^{2 \alpha\left(z \cdot \gamma^{-1} p\right)_{p}}, \quad\left(\frac{d \gamma_{\star} \nu}{d \nu}(z) \geq C^{-1} e^{-\alpha d\left(p, \gamma^{-1} p\right)} e^{2 \alpha\left(z \cdot \gamma^{-1} p\right)_{p}},\right)$

for all $\gamma \in \Gamma$ and some $C>1$, (where $\gamma_{\star} \nu=\gamma^{-1} \nu$ ). If both inequalities are satisfied, then we say $\nu$ is $\alpha$-quasiconformal. If in addition for each $\gamma \in \Gamma, \frac{d \gamma_{\star} \nu}{d \nu}$ is continuous, then $\nu$ is said to be a continuous $\alpha$-quasiconformal measure.

Suppose $\nu$ is $\alpha$-quasiconformal and the bounded function $R_{\gamma}$ given by

$$
R_{\gamma}(z)=\frac{d \gamma_{\star} \nu}{d \nu}(z) e^{\alpha d\left(p, \gamma^{-1} p\right)-2 \alpha\left(z \cdot \gamma^{-1} p\right)_{p}}
$$

satisfies for some $\epsilon>0$ and each $x \in \Lambda$,

$$
\sup _{\left\{y \mid 0<d(x, y)<e^{-\epsilon d\left(p, \gamma^{-1} p\right)}\right\}} \frac{\left|R_{\gamma}(x)-R_{\gamma}(y)\right|}{d_{p}^{\epsilon}(x, y)} \leq \frac{C}{e^{-\epsilon d\left(p, \gamma^{-1} p\right)}}
$$

for some $C$, independent of $\gamma$. Then we say that $\nu$ is Lipschitz $\alpha$-quasiconformal. All of the above definitions are independent of $p$.

Note that if $\nu$ is a continuous $\alpha$-quasiconformal measure with constant $C$ and $f: \partial X \rightarrow \mathbb{R}$ is a uniformly positive and bounded continuous function, then $f \nu$ is also a continuous $\alpha$-quasiconformal measure with constant $\frac{\sup f}{\inf f} C$.

The reason for the specific Lipschitz estimate in the definition of Lipschitz $\alpha$ quasiconformal should become clear in Section 4 For our purposes, assuming that $R_{\gamma}$ is Lipschitz uniformly in $\gamma$ would exclude the standard examples, while just assuming the Lipschitz constant depends arbitrarily on $\gamma$ is too weak.

Definitions 1.20. A family of finite Borel measure $\left\{m_{x}\right\}_{x \in X}$ of $\partial X$ is called an $f$-density of $\Gamma$ for a measurable function $f: X \times X \times \partial X \rightarrow \mathbb{R}$ if for all points $x, y \in \partial X$ the measure $m_{x}, m_{y}$ are equivalent with Radon-Nikodym derivatives

$$
\frac{d m_{y}}{d m_{x}}(z)=f(x, y, z)
$$

for $m_{x}$-a.e. $z \in \partial X$ and for all $\gamma \in \Gamma, f\left(\gamma^{-1} x, x, \gamma^{-1} z\right)=f(x, \gamma x, z)$. This is equivalent to the condition that $\gamma_{\star} m_{x}=m_{\gamma x}$. An $f$-density is called continuous 
if $f$ is continuous. An $f$-density is called bounded if the mass $\left\|m_{x}\right\|=m_{x}(\partial X)$ is bounded for all $x \in X$.

In what follows it is common to express quantities in terms of Busemann functions instead of the Gromov product, especially when $X$ is a "nice" space. To this end we introduce this notation here.

Definition 1.21. For $x, y \in X$ and $z \in \partial X$, we define the Busemann function $\rho_{x, z}$ by

$$
\rho_{x, z}(y)=2(x \cdot z)_{y}-d(x, y) .
$$

Since $d$ is $\Gamma$-invariant, we have

$$
\rho_{\gamma x, \gamma z}(\gamma y)=\rho_{x, z}(y)
$$

for all $x, y \in X, z \in \partial X$ and $\gamma \in \Gamma$.

Definition 1.22. If $f(x, y, z)=e^{-\alpha \rho_{x, z}(y)}$ for all $(x, y, z) \in X \times X \times \partial X$ and some $\alpha \in \mathbb{R}$, then the corresponding $f$-density is called an $\alpha$-conformal density of $\Gamma$.

Note that a single member $m_{p}$ of an $f$-density on $\Lambda(\Gamma)$ is an $\alpha$-quasiconformal density of $\Gamma$ if and only if there exists some constant $C \geq 1$ such that for all $\gamma \in \Gamma$ and $z \in \partial X$,

$$
C^{-1} e^{-\alpha \rho_{p, z}\left(\gamma^{-1} p\right)} \leq f(p, \gamma p, z) \leq C e^{-\alpha \rho_{p, z}\left(\gamma^{-1} p\right)} .
$$

If this holds for every member of the $f$-density for a uniform $C$, then we say that the $f$-density is an $\alpha$-quasiconformal density of $\Gamma$.

Now we turn our attention to two examples of $\alpha$-quasiconformal densities.

1.5.1. Hausdorff measures. Let $(Z, \delta)$ be any metric space and $D \geq 0$ be a nonnegative constant. Let $A$ be a subset of $Z$. For each $\epsilon \geq 0$ we define

$$
H_{\epsilon}^{D}(A) \triangleq \inf \left\{\sum_{j=1}^{\infty} r_{j}^{D} \mid A \subset \cup_{j} B\left(x_{j}, r_{j}\right), r_{j} \leq \epsilon, x_{j} \in A\right\}
$$

where the infinum is taken among all coverings of $A$ by balls of radius no more than $\epsilon$. The limit measure $\mathcal{H}_{\delta}^{D}(A)=\lim _{\epsilon \rightarrow 0} H_{\epsilon}^{D}(A)$ is called the $D$-dimensional Hausdorff measure of $A$. The Hausdorff dimension $\operatorname{dim}_{H}(A)$ is defined to be

$$
\operatorname{dim}_{H}(A) \triangleq \inf \left\{D: H_{\delta}^{D}(A)=0\right\}=\sup \left\{D: H_{\delta}^{D}(A)=\infty\right\} .
$$

In the definition of $\mathcal{H}_{\delta}^{D}$ we could have used any quasimetric bilipschitz to $\delta$ and obtained the same measure. Now we return to the $\delta$-hyperbolic setting. As a consequence of Item [5 in Proposition [1.6] for all $x, y \in X$ and $z \in \partial X$, the Busemann functions satisfy

$$
\left|\rho_{x, z}(y)+\rho_{y, z}(x)\right| \leq 4 \delta .
$$

Using this, we can now extend Proposition 4.3 in Coo93 to this more general class of $\delta$-hyperbolic spaces.

Proposition 1.23. Suppose $X$ is a $\delta$-hyperbolic space, and suppose $A \subset \partial X$ is a $\Gamma$-invariant Borel set with $0<\mathcal{H}_{\delta_{p}^{\epsilon}}^{\alpha / \epsilon}(A)<\infty$ for some $p \in X$. Then $\left\{\mathcal{H}_{\delta_{x}^{\epsilon}}^{\alpha / \epsilon}\right\}_{x \in X}$ is an $\alpha$-quasiconformal density of $\Gamma$ on $\partial X$. 
Proof. The quasi-antisymmetric property of Busemann functions described above allows us to rewrite the conclusion of Lemma 1.12 to obtain Lemma 2.2 of [Coo93, except using our definition of the Busemann function. Armed with this lemma, the proof of the proposition is identical to that of Proposition 4.3 in Coo93 in the geodesic setting.

1.5.2. Patterson-Sullivan measures. Assume that $\Gamma$ is discrete and acts properly dicontinuously on $X$. For two points $x, y \in X$ and for any real number $s>0$, we consider the Poincare series

$$
g_{s}(x, y)=\sum_{\gamma \in \Gamma} e^{-s d(x, \gamma y)} .
$$

Let $S_{k}$ be the number of the orbit points $\Gamma y$ in annulus $B\left(x, k+\frac{1}{2}\right)$

$B\left(x, k-\frac{1}{2}\right)$. Then $g_{s}(x, y)$ is proportional to $\sum_{k=0}^{\infty} S_{k} e^{-s k}$.

We define

$$
\delta(\Gamma) \triangleq \limsup _{k \rightarrow \infty} \frac{1}{k} \log S_{k}
$$

to be the critical exponent of $\Gamma$ which depends on the action of $\Gamma$ as much as $\Gamma$ itself. Then $g_{s}(x, y)$ diverges for $s<\delta(\Gamma)$ and converges for $s>\delta(\Gamma)$. So we can consider the family of measures

$$
\nu_{x}^{s}=\frac{1}{g_{s}(y, y)} \sum_{\gamma \in \Gamma} e^{-s d(x, \gamma y)} \delta_{\gamma y}, \quad s>\delta(\Gamma),
$$

where $\delta_{\gamma y}$ is the Dirac mass at $\gamma y$. Since

$$
d(x, \gamma y)-d(x, y) \leq d(y, \gamma y) \leq d(x, \gamma y)+d(x, y),
$$

we can easily see that $g_{s}\left(\gamma x, \gamma^{\prime} y\right)=g_{s}(x, y)$ for all $\gamma, \gamma^{\prime} \in \Gamma$ and

$$
e^{-s d(x, y)} g_{s}(x, y) \leq g_{s}(y, y) \leq e^{s d(x, y)} g_{s}(x, y) .
$$

Thus $\left\{\nu_{x}^{s}\right\}_{\delta(\Gamma)<s}$ is a family of finite measures on $X$ with total mass bounded by

$$
e^{-s d(x, \Gamma \cdot y)} \leq\left\|\nu_{x}^{s}\right\| \leq e^{-s d(x, \Gamma \cdot y)} .
$$

If $\liminf \inf _{s \rightarrow \delta(\Gamma)+} g_{s}(y, y)<\infty$, then we introduce a weighting function in front of the exponential factors above so that $\liminf _{s \rightarrow \delta(\Gamma)^{+}} g_{s}(y \cdot y)=\infty$. This can be done so that the essential properties of $\nu_{x}^{s}$ are preserved (see [Coo93 following [Pat76]). Let $\nu_{x}=\lim _{s_{j} \rightarrow \delta(\Gamma)+\nu_{x}^{s_{j}}}$ be a weak limit in the space of uniformly bounded measures on $X \cup \partial X$. There may be many distinct limit measures, however we always fix one such limit. Since $\lim _{s_{i} \rightarrow \delta(\Gamma)+} g_{s}(y . y)=\infty$, the measure $\nu_{x}$ is concentrated on the cluster points of the orbit $\Gamma y$, i.e., the limit set $\Lambda(\Gamma)$. From the construction it is easy to see that for any other point $y \in X$ the limit $\nu_{y}=\lim _{s_{j} \rightarrow \delta(\Gamma)^{+}} \nu_{y}^{s_{j}}$ also exists and that $\gamma_{\star} \nu_{x}=\nu_{\gamma x}$ for all $\gamma \in \Gamma$.

Suppose $\Gamma$ is not discrete or does not act properly discontinuously, but it admits a left-invariant infinite measure (or mean) $\eta$ with the property that for any compact set $K$ and any point $x \in X, \eta(\Gamma \cdot x \cap K)<\infty$. By converting the above sums to $\eta$-integrals we can still obtain a critical exponent $\delta(\Gamma)$ as well as the family of finite Patterson-Sullivan measures $\nu_{x}$.

In the case when $X$ is complete and geodesic, Coo93 shows that the RadonNikodym derivative at $z \in \partial X$ satisfies

$$
C^{-1} e^{-\delta(\Gamma) \rho_{x, z}(y)} \leq \frac{d \nu_{y}}{d \nu_{x}}(z) \leq C e^{-\delta(\Gamma) \rho_{x, z}(y)}
$$


for a constant $C \geq 1$ depending only on the hyperbolicity constant $\delta$. In short we have the following.

Corollary 1.24. If $X$ is complete and geodesic, then the family $\left\{\nu_{x}\right\}_{x \in X}$ of Patterson-Sullivan measures is an $\delta(\Gamma)$-quasiconformal density.

These measures are not automatically a continuous $\alpha$-quasiconformal density. However, if the Gromov product extends continuously to the boundary then both Busemann functions and a simple computation using the above formula for $\nu^{s}$ shows continuity for the Radon-Nikodym derivatives (see Coornaert [Coo93]). In the next section we shall see that, under still stronger conditions, these measures become $\alpha$-conformal.

1.6. The Shadow Lemma. Since our $\delta$-hyperbolic space is not necessarily a geodesic space, we cannot define a shadow for balls as in the Shadow Lemma proved by Sullivan. However, we can talk about the balls with respect to Busemann distance, even though it is not in general a metric. So we denote

$$
U_{p}(q)=\sup _{z \in \partial X}\left((z \cdot q)_{p}\right) \quad \text { and } \quad L_{p}(q)=\inf _{z \in \partial X}\left((z \cdot q)_{p}\right) .
$$

We abuse the notation and denote $U_{p, \gamma}=U_{p}\left(\gamma^{-1} p\right)$ and $L_{p, \gamma}=L_{p}\left(\gamma^{-1} p\right)$.

For every $p$ and $q$ we also fix a choice of points $z_{p, q}^{+}, z_{p, q}^{-} \in \partial X$ such that

$$
\left(z_{p, q}^{+} \cdot q\right)_{p} \geq U_{p}(q)-\delta \quad \text { and } \quad\left(z_{p, q}^{-} \cdot q\right)_{p} \leq L_{p}(q)+\delta .
$$

And for an isometry $\gamma$ we set $q=\gamma^{-1} p$ and $z_{p, q}^{+}=z_{p, \gamma}^{+}$and $z_{p, q}^{-}=z_{p, \gamma}^{-}$.

Lastly, we define $O_{p}(\gamma, D)=O_{p}\left(\gamma^{-1} p, D\right)$ where for $p, q \in X$ and $D \geq 0$,

$$
O_{p}(q, D):=\left\{y \in \partial X: d_{p}\left(z_{p, q}^{+}, y\right) \leq e^{-U_{p}(q)+D}\right\} .
$$

Remark 1.25. In the above notation the radial limit set can be written explicitly as

$$
\Lambda_{r}(\Gamma)=\bigcup_{D=0}^{\infty} \bigcap_{n=1}^{\infty} \bigcup_{\substack{\gamma \in \Gamma \\ d(p, \gamma p) \geq n}} O_{p}(\gamma, D) .
$$

Again, the right hand side is independent of the point $p$.

Lemma 1.26. (Shadow Lemma) Let $X$ be a $\delta$-hyperbolic space and $p \in X$. Assume $\nu\left(\right.$ or $\nu_{p}$ ) represents a finite measure that does not consist of a single atom.

a) If $X$ is weakly Gromov product bounded with respect to $\Gamma$, and $\nu$ is upper $\alpha$-quasiconformal, then there exist numbers $\beta>0$ and $D_{0} \geq 0$ such that for all $D>D_{0}$ and $\gamma \in \Gamma$ we have

$$
\nu\left(O_{p}(\gamma, D)\right) \geq \beta e^{-\alpha U_{p, \gamma}} \quad\left(\text { in particular, } \quad \nu\left(O_{p}(\gamma, D)\right) \geq \beta e^{-\alpha d\left(p, \gamma^{-1} p\right)-\alpha L_{p, \gamma}}\right) .
$$

b) If $X$ is upper Gromov product bounded with respect to $\Gamma$, and $\nu$ is lower $\alpha$-quasiconformal, then there exists a number $\beta>0$ such that for all $D \geq 0$ and $\gamma \in \Gamma$ we have

$$
\nu\left(O_{p}(\gamma, D)\right) \leq \beta e^{-\alpha U_{p, \gamma}} e^{2 \alpha D} \quad\left(\text { in particular, } \quad \nu\left(O_{p}(\gamma, D)\right) \leq \beta e^{-\alpha d\left(p, \gamma^{-1} p\right)+2 \alpha D}\right) .
$$


b') If $X$ is upper Gromov product bounded and $\left\{\nu_{x}\right\}_{x \in X}$ is a lower $\alpha$-quasiconformal density, then there exists a number $\beta>0$ such that for all $D \geq 0$ and $p, q \in X$ we have

$\nu_{p}\left(O_{p}(q, D)\right) \leq \beta\left\|\nu_{q}\right\| e^{-\alpha U_{p, q}} e^{2 \alpha D} \quad\left(\right.$ in particular, $\left.\quad \nu_{p}\left(O_{p}(q, D)\right) \leq \beta\left\|\nu_{q}\right\| e^{-\alpha d(p, q)+2 \alpha D}\right)$.

Proof. Without loss of generality we may assume $\nu$ is a probability measure and let the $C$ denote the quasiconformal constant for $\nu$.

For a), fix $\epsilon>0$ such that $d_{p}^{\epsilon}$ is a quasimetric. Since $\nu$ does not consist of a single atom, there exists a number $r>0$ and $a<1$ such that

$$
\nu(B(y, r))<a .
$$

By Lemma 1.13 for all $y \notin O_{p}(\gamma, D)$ we have

$$
e^{(y \cdot q)_{p}} \leq \frac{e^{3 \delta}}{d_{p}\left(z_{p}^{+}, q, y\right)} \leq e^{3 \delta} e^{\left(U_{p}(q)-D\right)} .
$$

Let $K$ be the constant for which $X$ is weakly Gromov product bounded relative to the point $p \in X$. Together with Lemma 1.14 we have,

$$
\begin{aligned}
d_{q}\left(z_{p, q}^{-}, y\right) & \leq d_{p}\left(z_{p, q}^{-}, y\right) e^{2 \delta} e^{-d(p, q)} e^{\left(\left(z_{p, q}^{-} \cdot q\right)_{p}+(y \cdot q)_{p}\right)} \leq \\
& \leq e^{2 \delta} e^{-d(p, q)} e^{\left(L_{p}(q)+\delta\right)} e^{3 \delta} e^{\left(U_{p}(q)-D\right)} \leq e^{6 \delta} e^{-(D-K)} .
\end{aligned}
$$

Thus $\operatorname{diam}_{d_{q}}\left(O_{p}(q, D)^{c}\right) \leq e^{6 \delta \epsilon} e^{-\epsilon(D-K)}$. Therefore setting $q=\gamma^{-1} p$, there exists $D_{0}$ such that for all $D \geq D_{0}$,

$$
\nu\left(\gamma O_{p}(\gamma, D)^{c}\right) \leq a \quad \text { or equivalently } \quad \nu\left(\gamma O_{p}(\gamma, D)\right) \geq 1-a .
$$

Now we have

$$
\begin{aligned}
1-a \leq & \nu\left(\gamma O_{p}(\gamma, D)\right)=\gamma_{\star}^{-1} \nu\left(O_{p}(\gamma, D)\right) \leq \int_{O_{p}(\gamma, D)} C e^{-\alpha d(p, q)} e^{2 \alpha(y \cdot q)_{p}} d \nu(y) \\
& \leq C e^{\alpha\left(2 U_{p, \gamma}-d\left(p, \gamma^{-1} p\right)\right)} \nu\left(O_{p}(\gamma, D)\right) .
\end{aligned}
$$

Thus we obtain that

$$
\nu\left(O_{p}(\gamma, D)\right) \geq(1-a) C e^{\alpha\left(d\left(p, \gamma^{-1} p\right)-U_{p, \gamma}\right)} e^{-\alpha\left(U_{p, \gamma}\right)} .
$$

To finish the proof recall that weak Gromov product bounded implies $U_{p, \gamma} \leq$ $d\left(p, \gamma^{-1} p\right)-L_{p, \gamma}+K$ for some constant $K>0$.

For part b), first note that for any $q \in X$ and every $y \in O_{p}(q, D)$ we can use Lemma 1.12 to obtain

$$
\left|(y \cdot q)_{p}-\left(z_{p, q}^{+} \cdot q\right)_{p}\right| \leq 2 \delta+D .
$$

This implies that

$$
e^{(y \cdot q)_{p}} \geq e^{-(2 \delta+D)} e^{\epsilon\left(U_{p, q}-\delta\right)}=e^{-D} e^{-3 \delta} e^{U_{p}(q)} .
$$

For every $D \geq 0$ and setting $q=\gamma^{-1} p$ we have

$$
\begin{aligned}
1 & \geq \gamma_{\star}^{-1} \nu\left(O_{p}(q, D)\right)=\int_{O_{p}(q, D)} d \gamma_{\star}^{-1} \nu(y)=\int_{O_{p}(q, D)} C^{-1} e^{-\alpha d(p, q)} e^{2 \alpha(y \cdot q)_{p}} d \nu(y) \\
& \geq C^{-1} e^{\alpha\left(2 U_{p}(q)-d(p, q)\right)} e^{-2 \alpha D} e^{-6 \alpha \delta} \nu\left(O_{p}(q, D)\right) .
\end{aligned}
$$


Using $d\left(p, \gamma^{-1} p\right) \leq U_{p, \gamma}+K$ we obtain the result. For part b') we simply replace the last estimate by

$$
\begin{aligned}
\left\|\nu_{q}\right\| & \geq \nu_{q}\left(O_{p}(q, D)\right)=\int_{O_{p}(q, D)} d \nu_{q}(y)=\int_{O_{p}(q, D)} C^{-1} e^{-\alpha d(p, q)} e^{2 \alpha(y \cdot q)_{p}} d \nu_{p}(y) \\
& \geq C^{-1} e^{\alpha\left(2 U_{p}(q)-d(p, q)\right)} e^{-2 \alpha D} e^{-6 \alpha \delta} \nu_{p}\left(O_{p}(q, D)\right) .
\end{aligned}
$$

A consequence of the generalized Shadow lemma above is that it can be plugged into Coornaert's proof of Corollary 6.6 in Coo93 to obtain the following.

Corollary 1.27. If $X$ is Gromov product bounded with respect to $\Gamma$, then any $\alpha$-quasiconformal measure is finite and nonzero only if $\alpha \geq \delta(\Gamma)$.

For convex cocompact actions on a CAT(-1) space the the Shadow Lemma implies that the Patterson-Sullivan measures $\left\{\nu_{p}\right\}_{p \in X}$ are Ahlfors $Q$-regular: there exists a $C>0$ such that for all $x \in \mathrm{CH}(\Lambda)$ and $r<1$,

$$
C^{-1} r^{Q} \leq \nu_{p}(B(x, r)) \leq C r^{Q} .
$$

In general Gromov hyperbolic spaces this need not be the case. However, we do not need such a strong condition. We only need a simple decay condition on the measure expressed in terms of a singular integral. This will be taken care by the following lemma.

\section{Lemma 1.28.}

(1) If $\left\{\nu_{x}\right\}_{x \in X}$ is a lower $\alpha$-quasiconformal density with constant $C$ and $z_{p, q}^{+} \in$ $\partial X$ is any point such that $\left(z_{p, q}^{+}, q\right)_{p} \geq U_{p}(q)-\delta$, then

$$
\int_{X-O_{p}(q, 0)} \frac{1}{d_{p}\left(z_{p, q}^{+}, y\right)^{2 \alpha}} d \nu_{p}(y) \leq C\left\|\nu_{q}\right\| e^{\alpha d(p, q)+6 \alpha \delta} .
$$

(2) If $\nu$ is lower $\alpha$-quasiconformal with respect to $\Gamma$ with constant $C$ and $z_{p, \gamma}^{+} \in$ $\partial X$ is any point such that $\left(z_{p, \gamma}^{+}, \gamma^{-1} p\right)_{p} \geq U_{p, \gamma}-\delta$, then

$$
\int_{X-O_{p}(\gamma, 0)} \frac{1}{d_{p}\left(z_{p, \gamma}^{+}, y\right)^{2 \alpha}} d \nu(y) \leq C\|\nu\| e^{\alpha d\left(p, \gamma^{-1} p\right)+6 \alpha \delta} .
$$

Proof. By Lemma 1.13 and the choice of $z_{p, q}^{+}$we have

$$
e^{(y \cdot q)} \geq \frac{e^{-3 \delta}}{d_{p}\left(z_{p, q}^{+}, y\right)}
$$

Therefore we have,

$$
\begin{aligned}
& C^{-1} e^{-\alpha d(p, q)} \int_{X-O_{p}(q, 0)} \frac{e^{-6 \alpha \delta}}{d_{p}\left(z_{p, q}^{+}, y\right)^{2 \alpha}} d \nu_{p}(y) \\
& \quad \leq \int_{X-O_{p}(q, 0)} C^{-1} e^{-\alpha d(p, q)} e^{2 \alpha(y \cdot q)_{p}} d \nu_{p}(y) \\
& \quad \leq \int_{X-O_{p}(q, 0)} \frac{d \nu_{q}}{d \nu_{p}}(y) d \nu_{p}(y) \leq \nu_{q}\left(O_{p}(q, 0)^{c}\right) \leq \nu_{q}(X) .
\end{aligned}
$$

For the second statement we restrict to the case when $q=\gamma^{-1} p$ and replace $\nu_{p}$ and $\nu_{q}$ with $\nu$ and $\gamma_{\star}^{-1} \nu$ respectively. 
Recall that a measure is ergodic with respect to an action of $\Gamma$ if for any measurable $\Gamma$ invariant set $A$, either $\mu(A)=0$ or $\mu\left(A^{\complement}\right)=0$. The following result is Proposition 3.3.1 of Yue96 for the case of discrete group acting freely on a pinched negatively curved Hadamard manifold. We present his proof to show that it works in the hyperbolic setting as well.

Proposition 1.29. Any two $\alpha$-conformal densities coincide up to a constant multiple if and only if $\Gamma$ acts ergodically on $\Lambda$.

Proof. Consider two such conformal densities $\left\{\mu_{x}\right\}_{x \in H}$ and $\left\{\nu_{x}\right\}_{x \in H}$. If $\Gamma$ is ergodic with respect to the measure class of $\mu$, consider the measure $\sigma=\frac{1}{2}(\mu+\nu)$ which is clearly also an $\alpha$-conformal density. Since $\mu_{x}$ and $\nu_{x}$ are both absolutely continuous to $\sigma_{x}$, their Radon- Nikodym derivatives $\frac{d \mu_{x}}{d \sigma_{x}}, \frac{d \nu_{x}}{d \sigma_{x}}$ exist and are $\Gamma$-invariant. By the ergodicity of these derivatives are equal to positive constants $\mu_{x}, \nu_{x}$-almost everywhere.

If $\Gamma$ is not ergodic with respect to the measure class of $\mu$, then there exists a Borel $\Gamma$-invariant subset $A \subset \Lambda$ such that for all $x \in H, \mu_{x}(A)>0$ and $\mu_{x}\left(A^{\mathrm{C}}\right)>0$. Defne $\sigma_{x}(E)=\mu_{x}(E \cap A)$; then $\sigma_{x}$ is another $\alpha$-conformal density.

\section{Metrics and measures on the boundary of a CAT(-1) SPACE}

In this section we describe some improvements that can be made on the the previous section if we restrict our attention to the case when $X$ belongs to the family of CAT $(-\kappa)$ spaces for $\kappa>0$. These are a special class of $\delta$-hyperbolic spaces that are uniquely geodesic, proper and for which the Gromov product extends continuously to the boundary. We recall definitions and few simple lemmas related to such spaces from BH99. It is no less general to assume $\kappa=1$ since this can be achieved by simply rescaling the metric. For the remainder of this section, let $H$ be a CAT(-1) space with metric $d$ and assume $\Gamma$ is a (nonelementary) discrete isometry group acting on $H$ properly discontinuously.

2.1. Busemann functions revisited. Let $\partial H$ be the ideal boundary of $H$. Again denote by $\Lambda$ the limit set of $\Gamma$ on $\partial H$, and $\Lambda_{r}$ the radial limit set of $\Gamma$ on $\partial H$.

For $x, y \in H$ and $z \in \partial H$, the Busemann function $\rho_{x, z}$ takes on the simpler expression:

$$
\rho_{x, z}(y)=\lim _{z_{i} \rightarrow z}\left(d_{H}\left(y, z_{i}\right)-d_{H}\left(x, z_{i}\right)\right) .
$$

It is easy to observe that

$$
\rho_{x, z}(y)-\rho_{x^{\prime}, z}(y)=\rho_{x, z}\left(x^{\prime}\right),
$$

for all $x, x^{\prime}, y \in H$ and $z \in \partial H$. Moreover, $\rho$ is continuous on $H \times H \times \partial H$ (Bou95). One important consequence of these facts is that we obtain an exact formula ([Bou95]) for the Radon-Nikodym derivative of the Patterson-Sullivan measures:

$$
\frac{d \nu_{y}}{d \nu_{x}}(z)=e^{-\delta(\Gamma) \rho_{x, z}(y)}
$$

In particular, we have the following.

Corollary 2.1. The family $\left\{\nu_{x}\right\}_{x \in H}$ form a continuous $\alpha$-conformal density on $\partial H$. 
2.2. Metrics on the boundary $\partial H$. Throughout this subsection we will fix a point $p \in H$ and $\epsilon>0$. We introduce two classes of metrics on the $\partial H$. Even though they are equivalent, i.e. bilipschitz, it will be convenient to use both of them for later results.

Busemann metric: The Busemann metric is defined to be

$$
d_{p}^{\epsilon}(z, y) \triangleq e^{-\frac{1}{2} \epsilon\left(\rho_{x, z}(p)+\rho_{x, y}(p)\right)}
$$

where $x$ is any point on the geodesic connecting $z$ and $y$. It is not too difficult to check that this definition is independent of the choice of $x$. Moreover, untangling the definition of the Busemann function shows that $d_{p}^{\epsilon}=e^{-\epsilon(z \cdot y)_{p}}=d_{p}^{\epsilon}$ and so we will continue to denote it by $d_{p}^{\epsilon}$. However, in the case of a CAT $(-\kappa)$-space, this is a genuine metric for all $0<\epsilon \leq \sqrt{\kappa}$ (see Bou96).

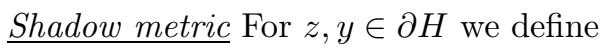

$$
\begin{gathered}
\ell_{p}(z, y) \triangleq \sup \left\{t \mid, d\left(\gamma_{p, z}(t), \gamma_{p, y}(t)\right) \leq 1\right\} \\
\operatorname{Sh}_{p}^{\epsilon}(z, y) \triangleq e^{-\epsilon \ell_{p}(z, y)},
\end{gathered}
$$

where $\gamma_{p, z}$ and $\gamma_{p, y}$ are the geodesics starting from $p$ and pointing to $z$ and $y$. This metric is called Shadow metric.

The following lemma, which states that these two metrics are equivalent, can be found in Kai90 for the pinched negatively curved manifold case. However, the proof works the same for CAT(-1) spaces as well.

Lemma 2.2. There exist $\epsilon_{0}>0$ such that $d_{p}^{\epsilon}(.,$.$) and S h_{p}^{\epsilon}(.,$.$) are distances for all$ $0<\epsilon \leq \epsilon_{0}$ and $p \in H$. Moreover these metrics are bilipschitz for the same $\epsilon$ : there is a $C>0$ such that for all $z, y \in \partial H$,

$$
C^{-1} \leq \frac{d_{p}^{\epsilon}(z, y)}{S h_{p}^{\epsilon}(z, y)} \leq C .
$$

Remark 2.3. For the sake of comparison to the existing literature on negatively curved spaces, we point out that there exist several other natural metrics on $\partial H$ which lie in the same bilipschitz class as the ones above. They include various explicit metrics under the name "Gromov's metric," and others with names like the "geodesic metric," the "horospherical metric," etc.... We omit their definitions since they will be unecessary for the discussion of this paper, but for many constructs we could have used them instead. However, the next lemma shows that $d_{p}^{\epsilon}$ is preferable in the CAT(-1) category.

Lemma 2.4. Each isometry $\gamma$ on $H$ induces a conformal map on $\partial H$ under the metric $d_{p}^{\epsilon}$.

Remark 2.5. This lemma justifies the previous notation of an $\alpha$-(quasi)conformal density. We are requiring that such a measure transform in a (quasi)conformal way under the family $\Gamma$ of conformal maps with respect to this metric.

Proof. (see Yue96.) The proof is almost the same as in the case of negatively curved manifolds, but we will repeat it for completion and to help the reader to get used to this notation.

Observe that for any $x, y \in H$ and $z \in \partial H$ and $\gamma \in \Gamma$ we have

$$
\rho_{x, \gamma z}(\gamma y)-\rho_{x, z}(y)=\rho_{\gamma^{-1} x, z}(y)-\rho_{x, z}(y)=\rho_{\gamma^{-1} x, z}(x) .
$$


So for $p, q \in X$ and $z, y \in \partial H$ we have

$$
\frac{d_{q}^{\epsilon}(z, y)}{d_{p}^{\epsilon}(z, y)}=\frac{e^{-\epsilon(y \cdot z)_{q}}}{e^{-\epsilon(y \cdot z)_{p}}}=e^{\epsilon\left((y \cdot z)_{p}-(y \cdot z)_{q}\right)}=e^{\epsilon\left((y \cdot q)_{p}+(z \cdot q)_{p}-d(p, q)\right)},
$$

by continuity of the Gromov product.

Now since the topology on $\partial H$ is inherited from the Gromov product, for each $a>0$ there exists a neighborhood $V_{a}$ of $z$ in $\partial H$ such that for all $y \in V_{a}$ we have

$$
(1-a) \leq e^{\epsilon\left((y \cdot q)_{p}-(z \cdot q)_{p}\right)} \leq(1+a) .
$$

So we proved that for every $a>0$ there exists a neighborhood $V_{a} \subset \partial H$ of $z$, such that for all $y \in V_{a}$ we have

$$
(1-a) e^{\epsilon\left(2(z \cdot q)_{p}-d(p, q)\right)} \leq \frac{d_{q}^{\epsilon}(z, y)}{d_{p}^{\epsilon}(z, y)} \leq(1+a) e^{\epsilon\left(2(z \cdot q)_{p}-d(p, q)\right)} .
$$

This proves that

$$
\lim _{y \rightarrow z} \frac{d_{q}^{\epsilon}(z, y)}{d_{p}^{\epsilon}(z, y)}=e^{\epsilon\left(2(z \cdot q)_{p}-d(p, q)\right)}=e^{-\epsilon \rho_{p, z}(q)} .
$$

The propositions and proofs for the rest of this section are taken from Yue96. which work just as well in the CAT(-1) setting as for negatively curved Hadamard manifolds.

First we present the analogue of Proposition 1.23. It occurs as Proposition 3.1.1 of Yue96 in the case $H$ a pinched negatively curved manifold. However, it easily follows by the same proof using Lemma 2.4 in the current setting of a CAT $(-1)$ space.

Proposition 2.6. If $A \subset \partial H$ is a $\Gamma$-invariant Borel set with $0<\mathcal{H}_{d}^{\alpha / \epsilon}(A)<\infty$ for any quasimetric $d$ bilipschitz to $d_{p}^{\epsilon}$ and $\alpha>0$, then $\left\{\mathcal{H}_{d_{p}^{\epsilon}}^{\alpha / \epsilon}\right\}_{p \in H}$ is a continuous $\alpha$-conformal density of $\Gamma$.

Next we collect other pertinent results which, except where indicated below, do not depend on the discreteness of $\Gamma$.

Proposition 2.7 (Theorem A, Corollaries 3.5.3 and 3.5.6 of Yue96]). If $\left\{\sigma_{x}\right\}_{x \in H}$ is any finite $\alpha$-conformal density for $\Gamma$ then $\alpha \geq \operatorname{dim}_{H}\left(\Lambda_{r}\right)$ and the following are equivalent for any $p \in H$,

(1) $\sigma_{p}\left(\Lambda_{r}\right)>0$

(2) $\Lambda_{r}$ has full $\sigma_{p}$ measure

If $\Gamma$ is discrete and properly discontinuous then these are equivalent to

(3) The Poincarè series $\sum_{\gamma \in \Gamma} e^{-\alpha d\left(p, \gamma^{-1} p\right)}$ diverges.

Moreover, if any of these conditions hold then $\alpha=\delta(\Gamma)=\operatorname{dim}_{H}\left(\Lambda_{r}\right)=\operatorname{dim}_{H}(\Lambda)$.

If $\Gamma$ is a discrete group acting convex cocompactly, then $\Lambda_{r}=\Lambda$ so finite nonzero $\delta(\Gamma)$-quasiconformal measures exist. Hence, that the Hausdorff measures $\mathcal{H}_{p}^{\delta(\Gamma)}$ and the Patterson-Sullivan measures $\nu_{p}$ (regardless of the choice of weak limit) coincide up to a constant multiple for each $p \in X$. In particular, all of the properties listed in Proposition 2.7 hold. 


\section{Regularity of MEASURES AND COVERS}

We first present some necessary notation. Recall that on a set $X$, a nonnegative function $d: X \times X \rightarrow \mathbb{R}$ is called a quasimetric (or quasidistance) if $d$ is symmetric, zero precisely along the diagonal, and satisfies the quasitriangle inequality:

$$
d(x, y) \leq C(d(x, z)+d(z, y))
$$

for some $C \geq 1$ and all $x, y, z \in X$.

3.1. Doubling and related properties. Let $X$ be a space equipped with a quasidistance function $d(\cdot, \cdot)$ and probability measure $\nu$. $\|\cdot\|$ denotes $L^{1}(X, \nu)$-norm.

Definition 3.1. (1) We say that a measure $\nu$ has $(p, \alpha)$-decay if there is a constant $D_{\nu}$ such that:

$$
\int_{X-B(x, r)} \frac{1}{d(y, x)^{p+\alpha}} d \nu(y) \leq \frac{D_{\nu}}{r^{p}}
$$

for every $x \in X$ and $1 \geq r>0$. We replace the right hand side by $D_{\nu}(1+|\log (r)|)$ if $p=0$ and we replace $X$ by $B(x, 1)$ if $p+\alpha<0$.

(2) We say that the measure $\nu$ has upper $Q$-regularity if there exists a constant $K_{\nu}>0$ such that the following is satisfied:

$$
\nu(B(x, r)) \leq K_{\nu} r^{Q}
$$

for every $x \in X$ and $r>0$.

(3) We say that the measure $\nu$ has the strong doubling property if there exists a constant $T_{\nu}$ such that

$$
\nu(B(x, 3 r)) \leq T_{\nu} \nu(B(x, r))
$$

for all $x \in X$ and $r>0$.

Remark 3.2. Note that if $\nu$ has the strong doubling property then to verify that $\nu$ has $(p, \alpha)$ decay, it is enough to verify condition (3.1) for $r \in\left\{1, c, c^{2}, c^{3}, \ldots\right\}$ for any $0<c<1$. Any other radius can be interpolated.

Lemma 3.3. Assume that $\nu$ is a finite measure with upper $Q$-regularity for some $Q \geq 0$. Then $\nu$ has $(p, \alpha)$-decay for all $p \geq 0$ and $\alpha \in \mathbb{R}$ satisfying $-p \leq \alpha \leq Q$.

Proof. First observe that if $p+\alpha \geq 0$, then

$$
\int_{X-B(x, r)} \frac{1}{d(y, x)^{p+\alpha}} d \nu(y) \leq \nu(X-B(x, 1))+\int_{B(x, 1)-B(x, r)} \frac{1}{d(y, x)^{p+\alpha}} d \nu(y)
$$


Without loss of generality, we may assume $r=\frac{1}{N}$ for $N>>1$, then

$$
\begin{aligned}
\int_{B(x, 1)-B(x, r)} \frac{1}{d(y, x)^{p+\alpha}} d \nu(y) & \leq \sum_{n=1}^{N} \frac{1}{(n r)^{p+\alpha}}(\nu(B(x,(n+1) r))-\nu(B(x, n r))) \\
& \leq \sum_{n=1}^{N}\left(\frac{1}{(n r)^{p+\alpha}}-\frac{1}{((n+1) r)^{p+\alpha}}\right) \nu(B(x,(n+1) r)) \\
& \leq \sum_{n=1}^{N}\left(\frac{1}{(n r)^{p+\alpha}}-\frac{1}{((n+1) r)^{p+\alpha}}\right) K_{\nu}((n+1) r)^{Q} \\
& \leq \sum_{n=1}^{N}\left(\frac{(n+1)^{Q}}{(n)^{p+\alpha}}-\frac{(n+1)^{Q}}{((n+1))^{p+\alpha}}\right) \frac{K_{\nu} r^{Q-\alpha}}{r^{p}} .
\end{aligned}
$$

We can estimate, under the assumption that $p+\alpha \geq 0$,

$\frac{(n+1)^{Q}}{(n)^{p+\alpha}}-\frac{(n+1)^{Q}}{((n+1))^{p+\alpha}}=\frac{(n+1)^{p+\alpha}-n^{p+\alpha}}{(n)^{p+\alpha}(n+1)^{p+\alpha-Q}} \leq C_{p+\alpha} \frac{n^{p+\alpha-1}}{(n)^{p+\alpha}(n+1)^{p+\alpha-Q}} \leq \frac{C_{p+\alpha}^{\prime}}{n^{p+\alpha-Q+1}}$,

for some constants $C_{p+\alpha}, C_{p+\alpha}^{\prime}$ and all $n \geq 1$. (We may take $C_{p+\alpha}^{\prime}=C_{p+\alpha}$ when $p+\alpha \geq Q$.) Therefore, if $p+\alpha \neq Q$, the above sum becomes

$$
\begin{aligned}
\frac{K_{\nu} r^{Q-\alpha}}{r^{p}} \sum_{n=1}^{N} \frac{C_{p+\alpha}^{\prime}}{n^{p+\alpha-Q+1}} & \leq \frac{K_{\nu} C_{p}^{\prime}}{r^{p}} \frac{1}{N^{Q-\alpha}}\left(\frac{N^{Q-p-\alpha}-1}{Q-p-\alpha}\right) \\
& \leq \frac{K_{\nu} C_{p+\alpha}^{\prime}}{r^{p}}\left(\frac{N^{-p}-N^{\alpha-Q}}{Q-p-\alpha}\right) .
\end{aligned}
$$

The quantity $\left(\frac{N^{-p}-N^{\alpha-Q}}{Q-p-\alpha}\right)$ is bounded for $p \geq 0$ whenever $Q-\alpha \geq 0$. When $p+\alpha=Q$ we may bound the sum by $1+\log (1 / r)$, obtaining the same conclusion. By using logarithmic bounds, the same conclusion is obtained in the case $p+\alpha=Q$. (Note we obtain the desired estimate $K_{\nu} C_{p+\alpha}^{\prime}(1+\log (1 / r))$ in the case $p+\alpha=Q$ and $p=0$.)

Remarks 3.4. The above proof also shows that if $\nu$ is any measure with $(p, \alpha)$-decay, then it also has $(p+t, \alpha)$-decay for all $t \geq 0$.

If $X$ has Hausdorff dimension $Q$ and $\nu$ is the corresponding Hausdorff measure, then $\nu$ has the strong doubling and $(p, \alpha)$-decay properties for all $p>0$ and $\alpha \leq Q$. However, $\nu$ may not be upper $Q$-regular.

3.2. Lipschitz constants. We shall say that a map $f: X \rightarrow Y$ between quasimetric spaces $X$ and $Y$ is locally Lipschitz if for every $r>0$ and $x \in X$ we have,

$$
\sup _{\substack{y \in B(x, r) \\ y \neq x}} \frac{d_{Y}(f(x), f(y))}{d_{X}(x, y)}<\infty .
$$

The various extant definitions of this notion for the most part agree when $X$ is proper. We now recall the definition of the Lipschitz constant on a given scale. 
Definition 3.5. For a locally Lipschitz map $f: X \rightarrow Y$, we define the Lipschitz constant at $x$ of scale $r$ to be the quantity,

$$
D_{r} f(x)=\sup _{\substack{y \in B(x, r) \\ y \neq x}} \frac{d_{Y}(f(x), f(y))}{d_{X}(x, y)} .
$$

Remark 3.6. It is clear that if $f$ is locally Lipschitz and $s \leq r$, then $D_{s} f(x) \leq$ $D_{r} f(x)$.

In the case of locally Lipschitz functions to $\mathbb{R}$, we summarize any arithmetic relations we will need in the following lemma. These will be mainly used in the proof of Theorem 6.1 Each case may be verified by a simple (and omitted) computation based on the definition.

Lemma 3.7. If $F, G$ are two locally Lipschitz functions on $X$, then $F+G$ and $F G$ are locally Lipschitz. Moreover,

(1) $D_{r}(F+G)(x) \leq D_{r} F(x)+D_{r} G(x)$,

(2) $D_{r}(c F)(x)=|c| D_{r} F(x)$, and

(3) $D_{r}(F G)(x) \leq\left(\sup _{d(x, y) \leq r}|F(y)|\right) D_{r} G(x)+\left(\sup _{d(x, y) \leq r}|G(y)|\right) D_{r} F(x)$.

(4) If $G(x) \neq 0$ for all $x \in X$, then $\frac{1}{G}$ is locally Lipschitz and

$$
D_{r}\left(\frac{1}{G}\right)(x) \leq \frac{D_{r} G(x)}{|G(x)|\left(\inf _{d(x, y) \leq r}|G(y)|\right)} .
$$

(5) If $H: Y \rightarrow X$ is locally Lipschitz, then

$$
D_{r}(F \circ H)(x) \leq D_{r * D_{r}(H)(x)}(F)(H(x)) * D_{r}(H)(x) .
$$

Because we will be using the operator $D_{r}$ with respect to (quasi)metrics which differ by taking powers, it will be convenient to quantitatively state the following well-known relationship.

Lemma 3.8. Let $F$ be a locally Lipschitz function on $X$ with respect to $d^{\epsilon^{\prime}}$ for some quasimetric $d$ and $\epsilon^{\prime}>0$. Choose any $\epsilon$ with $0<\epsilon \leq \epsilon^{\prime}$. If $D_{r}^{\epsilon} F$ and $D_{r}^{\epsilon^{\prime}} F$ represent the local Lipschitz constants of $F$ at scale $r$ with respect to quasimetrics $d^{\epsilon}$ and $d^{\epsilon^{\prime}}$ respectively, then

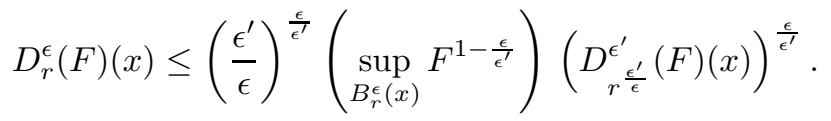

In particular, if $r \leq 1$ then

$$
D_{r}^{\epsilon}(F)(x) \leq 2\left(\sup _{B_{r}^{\epsilon}(x)} F^{1-\frac{\epsilon}{\epsilon^{\prime}}}\right)\left(D_{r}^{\epsilon^{\prime}}(F)(x)\right)^{\frac{\epsilon}{\epsilon^{\prime}}} .
$$

Here $B_{r}^{\epsilon}(x)$ is the $r$-ball centered at $x$ with respect to $d^{\epsilon}$.

Proof. The result follows by taking the supremum over $y \in B_{r}^{\epsilon}(x)$ of the estimate,

$$
\begin{aligned}
\frac{|F(x)-F(y)|}{d^{\epsilon}(x, y)} & \leq\left(\frac{\left|F(x)^{\frac{\epsilon^{\prime}}{\epsilon}}-F(y)^{\frac{\epsilon^{\prime}}{\epsilon}}\right|}{d^{\epsilon^{\prime}}(x, y)}\right)^{\frac{\epsilon}{\epsilon^{\prime}}} \\
& \leq\left(\frac{\epsilon^{\prime}}{\epsilon} \frac{|F(x)-F(y)|}{d^{\epsilon^{\prime}}(x, y)} \max \left\{F(x)^{\frac{\epsilon^{\prime}}{\epsilon}-1}, F(y)^{\frac{\epsilon^{\prime}}{\epsilon}-1}\right\}\right)^{\frac{\epsilon}{\epsilon^{\prime}}} .
\end{aligned}
$$


The second estimate comes from noting that $r^{\frac{\epsilon^{\prime}}{\epsilon}} \leq r$ and that $x^{-x} \leq 2$ for all $x>0$.

3.3. Besicovitch covers. Recall that a covering $\left\{U_{\alpha}\right\}_{\alpha \in \mathcal{A}}$ of a space $X$ has Lebesgue number $B \in \mathbb{N}$ if for every point $x \in X$,

$$
0<\#\left\{\alpha: x \in U_{\alpha}\right\} \leq B .
$$

Definition 3.9. A collection $\left\{U_{\alpha}\right\}_{\alpha \in \mathcal{A}}$ of subsets of a space $X$ is called a weak cover with Lebesgue number $B$ if $\nu$-almost every point of $X$ lies in at least one $U_{\alpha}$ and for all $x \in X$ we have

$$
\#\left\{\alpha: x \in U_{\alpha}\right\} \leq B
$$

Definition 3.10. We say that a collection $\left\{U_{\alpha}\right\}_{\alpha \in \mathcal{A}}$ where $U_{\alpha} \subset X$ is a Besicovitch cover, if there is a constant $B$ such that for every $\epsilon>0$, there exists a countable sub-collection $\left\{U_{\alpha_{i}}\right\}_{i=1}^{\infty} \subset\left\{U_{\alpha}\right\}_{\alpha \in \mathcal{A}}$ with $\operatorname{diam}\left(U_{\alpha_{i}}\right) \leq \epsilon$ which forms a covering with Lebesgue number $B$. We call a Besicovitch cover profinite if for each $\epsilon>0$, the corresponding subcover can be chosen to be finite. Similarly, we define a weak Besicovitch cover and a weak profinite Besicovitch cover as above, except that the subcollections are only expected to be weak covers with Lebesgue number $B$.

\section{SPIKES}

To keep the discussion as general as possible, in this section we assume that our measure is not a single atom and has support $X$.

Definition 4.1. Assume $\nu$ is a measure with $(Q, \theta)$ decay for some $Q \geq 0$ and $\theta \in \mathbb{R}$. A 6-tuple $(h(x), r, a, Q, \theta, C)$ where $h(x)$ is positive function on $X, r>0$, $C>1$ and $a \in X$, is called a spike if

(1) $h(x) \geq\|h\|_{L^{\infty}} / C$ on $B(a, r)$,

(2) for each $y \in B(a, r)^{c}$ we have

$$
0<h(y) \leq h(a) r^{Q} \int_{B(a, r)} \frac{C}{d(y, x)^{Q+\theta}} d \nu(x), \quad \text { and }
$$

(3) if $y, y^{\prime} \in X$ satisfy $d\left(y, y^{\prime}\right) \leq r$, then $h\left(y^{\prime}\right) \leq C h(y)$.

If $h(x)$ is a continuous function we call $(h(x), r, a, Q, \theta, C)$ a continuous spike. Also if $\|h\|_{L^{\infty}}=1$ we will call $(h(x), r, a, Q, \theta, C)$ a unit spike. Lastly we will often denote the spike by the function $h(x)$ alone with the other constants implicit.

Definition 4.2. If in addition a spike $(h(x), r, a, Q, \theta, C)$ has $h(x)$ locally Lipschitz with

$$
D_{r} h(x) \leq \frac{C h(x)}{r}
$$

for all $x \in X$, and

$$
\nu(B(a, r)) \geq \frac{r^{Q}}{C}
$$

then we call $h$ a $Q$-spike. 
Definition 4.3. For a spike $(h(x), r, a, Q, \theta, C)$ we call the number $\frac{\nu(B(a, 5 r))}{\nu(B(a, r))}$ the local doubling constant.

When working with families of spikes, we will denote the supremum of the local doubling constant over all spikes with final entry less than $C$ by $T_{\nu, C}$. Similarly, $T_{\nu}$ indicates the supremum of all local doubling constants. If for a given family of spikes we have $T_{\nu, C}<\infty$ for all $C>1$, then we say that members of this family are $\nu$-spikes.

Remark 4.4. Note that we implicitly assume that $a$ must lie in the support of $\nu$. For small families of spikes, it is much weaker to assume that the constant $T_{\nu}$ is bounded than to assume that $\nu$ has the strong doubling property.

It is clear that a positive multiple of a spike is a spike. Here are few simple Lemmas about spikes and $Q$-spikes. The first observation is immediate.

Lemma 4.5. Assume $(h, r, b, Q, \theta, C)$ is a spike. If $\frac{1}{M} f \leq A g \leq M f$ for some constants $A>0$ and $M \geq 1$, then $\left(g, r, b, Q, \theta, M^{2} C\right)$ is a spike.

Lemma 4.6. Assume $(h, r, b, Q, \theta, C)$ is a spike. Then

$$
\frac{\nu(B(b, r))}{C} \leq \frac{\|h\|_{L^{1}}}{\|h\|_{L^{\infty}}}
$$

Proof. Using the property 1) in the definition of a spike, we obtain

$$
\|h\|_{L^{1}} \geq \int_{B(b, r)} h d \nu \geq \nu(B(b, r)) \frac{\|h\|_{L^{\infty}}}{C} .
$$

Lemma 4.7. Assume that $(h, r, a, Q, \theta, C)$ is a spike. Then for all $t \geq 1,\left(h^{t}, r, a, Q, \theta, C^{t}\right)$ is a spike.

Proof. Observe that conditions 1),3) hold trivially. Condition 2) holds because $C^{t} \geq C \geq 1$, so taking the power in $t$ preserves the inequality.

Corollary 4.8. Assume that $(h, r, a, Q, \theta, C)$ is a $Q$-spike, then for all $t \geq 0$, $\left(h^{t}, r, a, Q, \theta, \max \left\{t C^{t}, C\right\}\right)$ is also a $Q$-spike.

Proof. We would like to use the following estimate. For all $a, b \in \mathbb{R}$ we have

$$
\left|a^{t}-b^{t}\right| \leq \max \{t, 1\}|a-b| \max \left(|a|^{t-1},|b|^{t-1}\right) .
$$

Now since $h(x)$ is a $Q$-spike, $h(x) \geq 0$. Also it is easy to see that for all $x, y \in X$ such that $d(x, y) \leq r$ we have $h(y)^{t-1} \leq c^{t-1} h^{t-1}(x)$. 
Thus we have

$$
\begin{aligned}
D_{r} h^{t}(x) & =\sup _{d(x, y) \leq r} \frac{\left|h^{t}(x)-h^{t}(y)\right|}{d(x, y)} \\
& \leq \sup _{d(x, y) \leq r} \max \{t, 1\} \frac{|h(x)-h(y)|}{d(x, y)} \max \left(h^{t-1}(x), h^{t-1}(y)\right) \leq \\
& \leq \max \{t, z\} C \frac{h(x)}{r} C^{t-1} h^{t-1}(x) \leq C^{t} \max \{t, 1\} \frac{h^{t}(x)}{r} .
\end{aligned}
$$

Now if $t<1$ then $C>C^{t}$ so in all cases $\max \left\{C, t C^{t}\right\} \geq C^{t} \max \{t, 1\}$. Moreover, this constant is always at least as large as $C$ so that the measure condition in the definition of the $Q$-spike $h$ persists.

Lemma 4.9. If $(h, r, a, Q, \theta, C)$ is a $Q$-spike and $f$ is any Lipschitz function with $\frac{1}{K} \leq f \leq K$ and $D_{r} f \leq \frac{K}{r}$, then $\left(f h, r, a, Q, \theta, 2 K^{2} C\right)$ is also a $Q$-spike.

Proof. By Lemma 4.5 it is a spike. Using the properties of $f$ and the third property of the spike $h$, we have

$$
\begin{aligned}
D_{r}(f h)(x) & \leq(\sup f) D_{r} h+(\sup h) D_{r} f \\
& \leq K \frac{C h(x)}{r}+C h(x) \frac{K}{r} \\
& \leq 2 K^{2} C \frac{h(x) f(x)}{r} .
\end{aligned}
$$

Since $h$ was a $Q$-spike this completes the proof.

\section{BASIS}

The purpose of this section is to prove the main ingredient for the coarse version of the stationarity result. This theorem provides a general criterion for the closure of the positive cone on a family of continuous positive functions $\left\{f_{i}\right\}$ on a metric measure space to be as large as possible in $L^{1}$. Here the closure is with respect to either $L^{1}$, uniform, or pointwise convergence. To the best of our knowledge, this is the first such general condition. We also indicate some examples showing that the hypotheses are in some aspects nearly sharp.

We call $(X, d, \nu)$ a probability metric space or pm space if $\nu$ is a Radon probability measure (with respect to the topology induced by $d$ ) of full support on $X$. We will also assume $X$ is separable, though not necessarily complete. (N.B. the Radon condition on $\nu$ could be replaced by the condition that $\nu$ is Borel and for all measurable sets $S, \nu(S)=\sup \{\nu(K): K \subset S$ compact $\}$.)

Definition 5.1. A function $F$ defined on a probability metric space $(X, d, \nu)$ is (almost) lower approximable if $F$ is (resp. almost everywhere) equal to the pointwise limit of a nondecreasing sequence of continuous functions.

Recall $F$ is (almost) lower semicontinuous, if $\liminf _{z \rightarrow x} F(z) \geq F(x)$ for every (resp. almost every) $x \in X$.

Finally, $F$ is (almost) uniformly positive if there is a $c$ such that $F(x) \geq c>0$ for every (resp. almost every) $x \in X$. The greatest (essential) lower bound for such an $f$ may be expressed as $\|1 / f\|_{-\infty}$.

Since $X$ is metrizable, it is perfectly normal. Hence the (almost) lower approximable functions are exactly the (almost) lower semicontinuous functions ([Ton52]). 
Theorem 5.2. Let $(X, d, \nu)$ be a probability metric space such that $\nu$ has $(Q, \theta)$ decay. Assume $\left\{\left(f_{\alpha}, r_{\alpha}, b_{\alpha}, Q, \theta, C_{\alpha}\right)\right\}_{\alpha \in \mathcal{A}}$ is a family of continuous unit $\nu$-spikes. For any $C>1$ let $S_{C}=\left\{\alpha \in \mathcal{A}: C_{\alpha} \leq C\right\}$ and set

$$
B_{C}(r)=\bigcup_{\substack{\alpha \in S_{C} \\ r_{\alpha} \leq r}} B\left(b_{\alpha}, r_{\alpha}\right) \quad \text { and } \quad B_{C}=\bigcap_{r>0} B_{C}(r) .
$$

If $\lim _{C \rightarrow \infty} \nu\left(B_{C}\right)=1$, then there exists a countable subset of indices $\left\{\alpha_{i}\right\}_{i=1}^{\infty} \subset \mathcal{A}$ such that for any uniformly positive almost lower semicontinuous function $F \in$ $L^{1}(X, \nu)$ there exists a sequence $\left\{\lambda_{\alpha_{i}}\right\}_{i=1}^{\infty}$ of nonnegative numbers such that $F=$ $\sum_{i=1}^{\infty} \lambda_{\alpha_{i}} f_{\alpha_{i}}$ for $\nu$-almost every $x \in X$ with convergence in $L^{1}$. Moreover, if $F$ is a lower semicontinuous (resp. continuous) function, then the convergence is pointwise (resp. uniform on compacta) on $\bigcup_{C>0} B_{C}$.

Remarks 5.3. Every function $F$ which can be expressed as a positive sum of the continuous functions $f_{\alpha}$ must be lower semicontinuous and positive, since ignoring the tail of the series yields an increasing sequence of positive continuous functions whose limit is therefore lower semicontinuous. In this sense the conclusion of the theorem is sharp. In fact, the theorem asserts that $\sum_{i=1}^{\infty} \lambda_{\alpha_{i}} f_{\alpha_{i}}$ will be the lower semicontinuous hull of $F$ for a general uniformly positive $F \in L^{1}(X)$. When $X$ is noncompact and the $f_{\alpha}$ are not uniformly positive, it is conceivable that some nonuniformly positive $F$ could be approximated as well. However, to address this case we would have had to make complicated compatibility assumptions on how and where $F$ and the $f_{\alpha}$ decay to 0 which we felt were not worth the extra effort.

Examples of almost lower semicontinuous functions include any $F$ whose set of points of discontinuity (or undefined points) $D \subset X$ has measure 0. Simply note that the lower semicontinuous hull $\tilde{F}$ of $F$ defined by

$$
\tilde{F}(x):= \begin{cases}F(x) & F(x) \text { exists and } F(x) \leq \liminf _{y \rightarrow x} F(y) \\ \liminf _{y \rightarrow x} F(y) & \liminf _{y \rightarrow x} F(y)<\infty \text { and } x \notin \operatorname{Dom}(F) \text { or } F(x)>\liminf _{y \rightarrow x} F(y) \\ 1 & \text { otherwise }\end{cases}
$$

agrees with $F$ almost everywhere.

One cannot remove the assumption that $\nu\left(\cup_{C>0} B_{C}\right)=1$, even if $\cup_{C>0} B_{C}$ is assumed to be dense, since an $L^{1}$ function with mass outside $\cup_{C>0} B_{C}$ could never be approximated in $L^{1}$. (Also, see the examples below.)

Lastly, the difficulty in proving the theorem reflects a certain balancing act captured by the spike conditions. While it may be possible to weaken these, we briefly mention why these conditions are qualitatively necessary. Since there can be no cancellation in the sum, it is evident that the positive basis functions must contain subsequences which, when suitably normalized, converge to Dirac distributions based at almost every point. However the shape of the $f_{\alpha}$ are further constrained. If they all decay too quickly then any countable subset cannot generally approximate on a full measure set. If the $f_{\alpha}$ decay too slowly then their tails stack up too quickly far away from their maximum. This too prevents generic approximation. In fact, it is somewhat surprising that the spike conditions happen to be satisfied for the Radon-Nikodym derivatives of the geometric measures in our main application (see Section 7). 
Example 5.4. Uniformly bounded upper semicontinuous functions need not be almost lower semicontinuous. For instance, let $X=[0,1]$ and define $F=F_{C}$ to take value 2 on a Cantor set $C$ of positive Lebesgue measure, and have value 1 elsewhere. Any lower continuous approximation must lie below 1, otherwise all closer approximations are greater than $F$ by a fixed positive amount on an open set, a contradiction. Either way, this prevents the limit from converging in $L^{1}$ to $F_{C}$. Nevertheless, $F_{C}$ is upper semicontinuous since it is the pointwise limit of a nonincreasing sequence of continuous approximations to the step functions which take the value 2 on the set of intervals representing the $n$th stage in the construction of the Cantor set and take the value 1 elsewhere. The same argument shows that the conclusion of the theorem holds for many functions with a positive measure set of discontinuities, for instance, $F=3-F_{C}$.

Example 5.5. In this example we present the simplest case to which we will apply the theorem. It also motivates the definition of a spike and further indicates why the hypothesis on the balls $B\left(b_{i}, r_{i}\right)$ is necessary. Consider $\mathbb{H}^{2}$ in the disk model. Let $\Gamma$ be a discrete group of $\operatorname{Isom}\left(\mathbb{H}^{2}\right)$ and for each $\gamma \in \Gamma$ let $(d, \theta)=\left(d_{\gamma}, \theta_{\gamma}\right)$ represent the orbit point $\gamma \cdot 0$ where $d$ is its hyperbolic distance from 0 and $\theta$ is its angle from the real axis. Then if $\phi$ is the angle coordinate on $\partial \mathbb{H}^{2}=S^{1}$, we may write the Poisson kernel based at $\gamma \cdot 0$ normalized to have maximum 1 as

$$
f_{\gamma}(\phi)=\frac{1}{e^{d}(\cosh (d)-\cos (\theta-\phi) \sinh (d))} .
$$

If $r_{\gamma}$ represents the radius on $S^{1}$ of the points where $f_{\gamma}>1 / C$, then $r_{\gamma}=$ $\arccos (C-(C-1) \operatorname{coth}(d))$. We will show in Section 7 that if $\nu$ is the Lebesgue probability measure on $S^{1}$, then the tuples $\left(f_{\gamma}, \theta_{\gamma}, r_{\gamma}, 1,1, C\right)$ for all $\gamma \in \Gamma$ and $C=2,3, \ldots$ form a family of continuous unit $\nu$-spikes. We will see that for the family of balls $B\left(\theta_{\gamma}, r_{\gamma}\right)$ and sets $B_{C}$ as in the theorem, the radial limit set of $\Gamma$ coincides with $\cup_{C>1} B_{C}$. However, it is easy to construct examples of $\Gamma$ such that their radial limit set has measure zero and hence $\lim _{C \rightarrow \infty} \nu\left(B_{C}\right)=0$. For such a group $\Gamma$, any function $F>0$ approximated by any positive sums of $f_{\gamma}$ must have more than $1 / 2$ of its $L^{1}$ norm concentrated on $\cup_{C>1} B_{C}$ since each term in the sum does. Of course one can provide a work around for this obstruction by simply restricting the measure to the limit set. This can be made to work so long as the radial limit set has full measure in the limit set.

This case of the theorem for uniform approximations of continuous functions on the circle by Poisson kernels was first proved by Hayman and Lyons ( HL90, see also [BW89]) using the theory of harmonic functions. That result was later extended to Euclidean domains in Gar96.

Remark 5.6. An analysis of the proof of the next proposition shows that while the condition of the $\nu$-spike is probably not absolutely necessary for a positive basis, it is a very natural condition which, up to small possible improvements, is necessary for the intuitive approach we take.

The proof of Theorem 5.2 will require the following proposition.

Proposition 5.7. Let $(X, d, \nu)$ be a probability metric space such that $\nu$ has $(Q, \theta)$ decay. Assume $\left\{\left(f_{i}(x), r_{i}, b_{i}, Q, \theta, C_{i}\right)\right\}$ is a countable family of unit $\nu$-spikes on $X$ with bounded doubling constants and such that $C_{i} \leq C$. Let $Y \subset X$ be a set weakly covered by $\left\{B\left(b_{i}, r_{i}\right)\right\}$ with finite Lebesgue number. 
For any positive function $F$ bounded away from 0 on $X$ and bounded from above on $Y$, set

$$
t=\left(\frac{\sup _{z \in Y}(F(z))}{\inf _{z \in X}(F(z))}\right)^{\frac{1}{Q}}+1 \geq 2 .
$$

Suppose for a given $s>1$ there is a $\delta>0$ such that

$$
s \geq \sup \left\{\frac{F(y)}{F(x)} \mid x \in X \text { and } y \in Y \text { with } d(x, y) \leq \delta\right\}
$$

and $r_{i} \leq \delta / t$ for all $i \in \mathbb{N}$. Then,

a. There exists a constant $0<L_{\nu}<1$, which do not depend on $F$, and a function $h=\sum_{i=1}^{\infty} \lambda_{i} f_{i}$ with each $\lambda_{i} \geq 0$ and only a finite number of the $\lambda_{i}$ not equal to 0 , such that for every $x \in X$

$$
h(x) \leq F(x)
$$

and

$$
\frac{L_{\nu}}{C^{2} s^{2}}\|F(x)\|_{L_{1}(Y)} \leq\|h(x)\|_{L_{1}(Y)} .
$$

b. If in addition $Y$ is (weakly) covered by a finite number of the $B\left(b_{i}, r_{i}\right)$, then

$$
\frac{L_{\nu}}{C^{2} s^{2}} F(x) \leq h(x)
$$

for ( $\nu$-almost) every $x \in Y$.

Proof of Proposition. In what follows, we let $T_{\nu}$ be the bound on the doubling constant. We let $B$ be the Lebesgue number of the cover, and we let $D_{\nu}$ be decay constant of $\nu$. Without loss of generality we also assume that $r_{i}$ is nonincreasing. Now we inductively build functions $g_{n}(x)$ as follows.

$$
\begin{aligned}
& g_{0}(x)=0 . \\
& g_{n}(x)=\left\{\begin{array}{lll}
g_{n-1}(x) & \text { if } \quad g_{n-1}\left(b_{n}\right) \geq F\left(b_{n}\right) \\
g_{n-1}(x)+f_{n}(x)\left(F\left(b_{n}\right)-g_{n-1}\left(b_{n}\right)\right) & \text { if } \quad g_{n-1}\left(b_{n}\right)<F\left(b_{n}\right)
\end{array}\right.
\end{aligned}
$$

This construction yields a sequence $\lambda_{1}, \lambda_{2}, \ldots$ with

$$
g_{n}(x)=\sum_{i=1}^{n} \lambda_{i} f_{i}(x)
$$

and $0 \leq \lambda_{i} \leq F\left(b_{n}\right)$. Hence, $g_{n}(x) \in V_{+}$.

Lemma 5.8. If $y^{\prime} \notin \cup_{i=1}^{n} B\left(b_{i}, r_{i}\right)$ and $d\left(y, y^{\prime}\right) \leq r_{n}$, then $g_{n}\left(y^{\prime}\right) \leq C g_{n}(y)$.

Proof. Since $d\left(y, y^{\prime}\right) \leq r_{n} \leq r_{i}$ for all $i \leq n$, by Remark above $f_{i}\left(y^{\prime}\right) \leq C f_{i}(y)$ for all $i=1, \ldots, n$. Since $g_{n}(x)=\sum_{i=1}^{n} \lambda_{i} f_{i}(x)$ with nonnegative coefficients, we obtain that $g_{n}\left(y^{\prime}\right) \leq C g_{n}(y)$.

Lemma 5.9. $g_{n}\left(y^{\prime}\right) \leq C g_{n}(y)+B s F\left(y^{\prime}\right)$, for all $y, y^{\prime} \in X$ and $d\left(y, y^{\prime}\right) \leq r_{n}$.

Proof. By the previous lemma we may assume that $y^{\prime} \in B\left(b_{k}, r_{k}\right)$, for some $k \leq n$. By construction $g_{n}(x)=\sum_{i=1}^{n} \lambda_{i} f_{i}(x)$, with $0 \leq \lambda_{i} \leq F\left(b_{i}\right)$. Thus for each $i \neq k$, we have $f_{i}\left(y^{\prime}\right) \leq C f_{i}(y)$.

For $i=k$, we have that $\lambda_{k} \leq F\left(b_{k}\right)$. However, since $d\left(y^{\prime}, b_{k}\right) \leq r_{k} \leq \delta$, we have that $F\left(b_{k}\right) \leq s F\left(y^{\prime}\right)$. Since $B$ is the Lebesgue number of the weak cover, $\#\left\{k \mid y^{\prime} \in B\left(b_{k}, r_{k}\right)\right\} \leq B$. Thus we conclude that $g_{n}\left(y^{\prime}\right) \leq C g_{n}(y)+B s F\left(y^{\prime}\right)$. 
Lemma 5.10. Let $L^{\prime}=s C+s B+C D_{\nu} B+s B T_{\nu}+s B C D_{\nu} 2^{Q}$. Then

$$
g_{N}(y) \leq L^{\prime} F(y)
$$

for all $N \in \mathbb{N}$ and $y \in X$.

Proof. Assume that $n$ is the smallest integer such that $g_{n}(y) \geq(s C+B s) F(y)$ for any $y \in X$. If there is no such $n$ then set $L^{\prime}=(s C+B s)$. Otherwise, we have $g_{n-1}(y)<(s C+B s) F(y)$ for all $y \in X$.

By Lemma 5.9 $g_{k}(y) \leq C g_{k}\left(y^{\prime}\right)+B s F(y)$ for all $k \in \mathbb{N}$ and $y^{\prime} \in X$ such that $d\left(y, y^{\prime}\right) \leq r_{k} \leq \delta$. Therefore, we obtain that $g_{n}\left(y^{\prime}\right) \geq s F(y) \geq F\left(y^{\prime}\right)$.

Thus if $d\left(y, b_{k}\right) \leq r_{n}$ and $k>n$, by construction we have $\lambda_{k}=0$.

Now since $\lambda_{i} f_{i}\left(b_{i}\right) \leq F\left(b_{i}\right)$ and by the property of spikes we have,

$$
\sum_{\substack{i \geq n, \delta \geq d\left(y, b_{i}\right), d\left(y, B\left(b_{i}, r_{i}\right)\right) \geq \frac{r_{n}}{2}}} \lambda_{i} f_{i}(y) \leq \sum_{\substack{i \geq n, \delta \geq d\left(y, b_{i}\right), d\left(y, B\left(b_{i}, r_{i}\right)\right) \geq \frac{r_{n}}{2}}} F\left(b_{i}\right) r_{i}^{Q} \int_{B\left(b_{i}, r_{i}\right)} \frac{C}{d(y, x)^{Q+\theta}} d \nu(x),
$$

and since $r_{i} \leq r_{n}$ and $F\left(b_{i}\right) \leq s F(y)$ we have,

$$
\leq s F(y) r_{n}^{Q} B \int_{X-B\left(y, \frac{r_{n}}{2}\right)} \frac{C}{d(y, x)^{Q+\theta}} d \nu(x),
$$

and by $(Q, \theta)$-decay this becomes,

$$
\leq s F(y) B C D_{\nu} 2^{Q}
$$

Now we consider $d\left(y, B\left(b_{i}, r_{i}\right)\right) \leq \frac{r_{n}}{2}$ for $i \geq n$. Therefore $B\left(b_{i}, r_{i}\right) \subset B\left(y, 3 / 2 r_{n}\right)$.

In case $r_{i} \leq r_{n} / 2$, then $d\left(y, b_{i}\right) \leq r_{n}$. As we observe above, in this case $\lambda_{i}=0$

So if $\lambda_{i} \neq 0$ and $d\left(y, B\left(b_{i}, r_{i}\right)\right) \leq \frac{r_{n}}{2}$ for $i \geq n$, we obtain that $r_{i} \geq r_{n} / 2$. Since $d\left(y, r_{i}\right) \leq r_{n}$ we have that $B\left(y, 3 / 2 r_{n}\right) \subset B\left(b_{i}, 5 r_{i}\right)$.

$$
\begin{aligned}
& \sum_{\substack{r_{i} \geq \frac{r_{n}}{2}, d\left(y, B\left(b_{i}, r_{i}\right)\right) \leq \frac{r_{n}}{2}}} \nu\left(B\left(b_{i}, r_{i}\right)\right) \leq B \nu\left(B\left(y, \frac{3 r_{n}}{2}\right)\right) \leq \\
& \leq \frac{B}{\#\left\{i: r_{i} \geq \frac{r_{n}}{2}, d\left(y, B\left(b_{i}, r_{i}\right)\right) \leq \frac{r_{n}}{2}\right\}} \sum_{\substack{r_{i} \geq \frac{r_{n}}{2}, d\left(y, B\left(b_{i}, r_{i}\right)\right) \leq \frac{r_{n}}{2}}} \nu\left(B\left(b_{i}, 5 r_{i}\right)\right) \leq \\
& \leq \#\left\{\sum_{\nu} \nu\left(B\left(b_{i}, r_{i}\right)\right) .\right. \\
&\left.\sum_{\substack{r_{i} \geq \frac{r_{n}}{2}, d\left(y, B\left(b_{i}, r_{i}\right)\right) \leq \frac{r_{n}}{2}}} \nu \frac{r_{n}}{2}, d\left(y, B\left(b_{i}, r_{i}\right)\right) \leq \frac{r_{n}}{2}\right\}
\end{aligned}
$$

Therefore

$$
\#\left\{i: r_{i} \geq \frac{r_{n}}{2}, d\left(y, B\left(b_{i}, r_{i}\right)\right) \leq \frac{r_{n}}{2}\right\} \leq T_{\nu} B
$$

Since $\lambda_{i} \leq F\left(b_{i}\right)$ and $f_{i}(y) \leq 1$, we obtain

$$
\sum_{\substack{\left.i \geq n, \delta \geq d\left(y, b_{i}\right), d y, B\left(b_{i}, r_{i}\right)\right) \leq \frac{r_{n}}{2}}} \lambda_{i} f_{i}(y) \leq \sum_{\substack{i \geq n, \delta \geq d\left(y, b_{i}\right), d\left(y, B\left(b_{i}, r_{i}\right)\right) \leq \frac{r_{n}}{2}}} s F(y) \leq s F(y) T_{\nu} B
$$


Also observe that

$$
\begin{aligned}
& \sum_{\substack{i \geq n, \delta \leq d\left(y, b_{i}\right)}} \lambda_{i} f_{i}(y) \leq \sum_{\substack{i \geq n, \delta \leq d\left(y, b_{i}\right)}} F\left(b_{i}\right) r_{i}^{Q} \int_{B\left(b_{i}, r_{i}\right)} \frac{1}{d(y, x)^{Q+\theta}} d \nu(x) \leq \\
\leq & \sup _{z}(F(z)) r_{n}^{Q} B \int_{X-B\left(y, \delta-r_{n}\right)} \frac{C}{d(y, x)^{Q+\theta}} d \nu(x) \leq \\
\leq & \frac{B C D_{\nu} \sup _{z}(F(z)) r_{n}^{Q}}{\left(\delta-r_{n}\right)^{Q}} \leq \frac{B C D_{\nu} \sup _{z}(F(z))(\delta / t)^{Q}}{(\delta-\delta / t)^{Q}} \leq \\
& \leq C D_{\nu} B \sup _{z}(F(z)) \frac{1}{(t-1)^{Q}}
\end{aligned}
$$

Now recall that

$$
t=\left(\frac{\sup _{z}(F(z))}{\inf _{z}(F(z))}\right)^{\frac{1}{Q}}+1
$$

So we obtain that

$$
\sum_{\substack{i \geq n, \delta \leq d\left(y, b_{i}\right)}} \lambda_{i} f_{i}(y) \leq C D_{\nu} B F(y) .
$$

So for these choices we obtain

$$
\begin{aligned}
& g_{N}(y)=\sum_{i=1}^{N} \lambda_{i} f_{i}(y)
\end{aligned}
$$

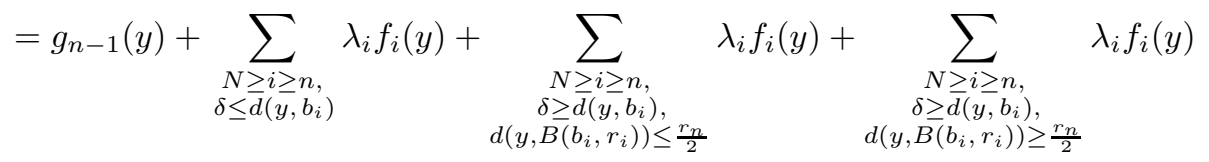

$$
\begin{aligned}
& \leq(s C+s B) F(y)+C D_{\nu} B F(y)+s F(y) B T_{\nu}+F(y) s B C D_{\nu} 2^{Q} \\
& =F(y)\left(s C+s B+C D_{\nu} B+s B T_{\nu}+s B C D_{\nu} 2^{Q}\right)
\end{aligned}
$$

Let $L^{\prime}=s C+s B+C D_{\nu} B+s B T_{\nu}+s B C D_{\nu} 2^{Q}>3$.

Set

$$
3 L_{\nu}=\frac{1}{1+B+D_{\nu} B+B T_{\nu}+B D_{\nu} 2^{Q}} .
$$

Since $C>1$ and $s \geq 1$ by Lemma 5.10 we have

$$
g_{N}(y)<\frac{C s}{3 L_{\nu}} F(y)
$$

for all $y \in X$.

Since $g_{N}(y)$ is bounded nondecreasing sequence, $\lim _{N \rightarrow \infty} g_{N}(y)=g(y)$ is welldefined.

Lemma 5.11. for every $y \in \cup_{n=1}^{\infty} B\left(b_{n}, r_{n}\right)$,

$$
g(y) \geq \frac{F(y)}{C s} .
$$


Proof. Assume that $n$ is the smallest integer such that $y \in B\left(b_{n}, r_{n}\right)$. By the spike properties, we have

By Lemma 5.8

$$
f_{n}(y) \geq \frac{1}{C}=\frac{f_{n}\left(b_{n}\right)}{C} .
$$

So we obtain that

$$
g_{n-1}(y) \geq \frac{1}{C} g_{n-1}\left(b_{n}\right) .
$$

$$
g_{n}(y) \geq \frac{g_{n}\left(b_{n}\right)}{C} .
$$

Again by construction $g_{n}\left(b_{n}\right) \geq F\left(b_{n}\right)$. Also as $d\left(y, b_{n}\right) \leq r_{n} \leq \delta$, we get $s F(y) \geq$ $F\left(b_{n}\right)$. Therefore,

So we conclude that

$$
g_{n}(y) \geq \frac{g_{n}\left(b_{n}\right)}{C} \geq \frac{F\left(b_{n}\right)}{C} \geq \frac{F(y)}{s C} .
$$

$$
g(y) \geq g_{n}(y) \geq \frac{F(y)}{s C} .
$$

Now define

$$
h_{n}(y)=\frac{3 L_{\nu} g_{n}(y)}{C s} \in V_{+}
$$

and $\lim _{n \rightarrow \infty} h_{n}(y)=h(y)$. By Lemma [5.10] $h(y) \leq \beta F(y)$ where $\beta=\frac{3 L_{\nu}}{C s} L^{\prime}<1$. Note that $\beta$ is bounded below by $3 L_{\nu}\left(1+B D_{\nu} 2^{Q}\right)$ independent of $C$ and $s$. By Lemma 5.11

for $\nu$-almost every point.

$$
h(y) \geq \frac{3 L_{\nu}}{C^{2} s^{2}} F(y)
$$

So

$$
\|h(x)\| \geq \frac{3 L_{\nu}\|F(x)\|_{L^{1}(Y)}}{C^{2} s^{2}}>\frac{L_{\nu}}{C^{2} s^{2}}\|F(x)\|_{L^{1}(Y)} .
$$

Since $\lim _{n \rightarrow \infty}\left\|h_{n}(x)\right\|=\|h(x)\|$ there exists $n$ with required property. This finishes part a).

For part b), it is easy to observe that if the cover is finite, there exists $N$ such that for all $n>N, h_{n}(y)=h(y)$. If $F$ is not locally constant then $t>1$ and therefore the conclusion of Lemma [5.11 is true on a uniform neighborhood of $\cup_{n=1}^{\infty} B\left(b_{n}, r_{n}\right)$ which includes all of $Y$ since a full measure subset was assumed to be dense. This finishes the proof of Proposition 5.7

Proof of Theorem 5.2. We begin by proving the first part of the theorem for the special case of uniformly positive continuous functions in $L^{1}$. We will find a universal countable subfamily of spikes indexed by $\left\{\alpha_{i}\right\}_{i=1}^{\infty} \subset \mathcal{A}$ so that for every such function $F$ we will inductively build a sequence $\left\{h_{i}(x)\right\}_{i=0}^{\infty} \subset V_{+}$, where $V_{+}=\left\{\sum_{i=1}^{n} a_{i} f_{\alpha_{i}}(x): a_{i} \geq 0, \alpha_{i} \in \mathcal{A}, n \in \mathbb{N}\right\}$, such that for some $0<\gamma_{n}<1$,

$$
\begin{aligned}
& 0<\sum_{i=0}^{n} h_{i}(x) \leq \gamma_{n} F(x) \quad \text { for all } x \in X \text { and } \\
& \left\|F-\sum_{i=0}^{n} h_{i}\right\|_{1} \rightarrow 0 \text { as } n \rightarrow \infty .
\end{aligned}
$$


Once this is achieved we can simply note that the sums $\sum_{i=1}^{n} h_{i}$ live in $V_{+}$and converge to $F$ in $L^{1}$ as desired.

Let $\left\{C_{n}\right\}$ denote any sequence tending to $\infty$. By hypothesis, the sequence $B_{C_{n}}$ exhaust a full measure subset of $X$. Since $\nu$ is a Radon measure, we may enclose the complement of $B_{C_{n}}$ by an open set $O_{n}$ with approximately the same measure. Since the complement of $O_{n}$ is a closed subset of $B_{C_{n}}$ we may find compact subsets $Y_{n} \subset B_{C_{n}}$ such that $\lim _{n \rightarrow \infty} \nu\left(Y_{n}\right)=1$.

To prove \&), first set $h_{0}(x)=0$. For the inductive step, assume we found $h_{0}(x), h_{1}(x), \ldots, h_{n}(x) \in V_{+}$such that

$$
0<\sum_{i=0}^{n} h_{i}(x) \leq \gamma_{n} F(x)
$$

Set $R_{n}(x)=F(x)-\sum_{i=0}^{n} h_{i}(x)$. Observe that $R_{n}(x)$ is a positive continuous function. Since $\gamma_{n}<1$ and $F$ is uniformly positive, $R_{n}$ is also uniformly positive. Since $F$ and each $h_{i}$ are uniformly continuous on $Y_{n}$, so is $R_{n}(x)$. Hence for $Y=Y_{n}$ and any fixed $s>1$ there exists a $\delta>0$ and $t<\infty$, both depending on $n$, that satisfies the first condition of Proposition 5.7 Now by Theorem 2.8.7 in [Fed69, for any $\epsilon>0$, there is a Vitaly cover of $B_{C_{n}}=\cap_{r>0} B_{C_{n}}(r)$ by a countable family of balls $\left\{B\left(b_{\alpha_{i, \epsilon, n}}, r_{\alpha_{i, \epsilon, n}}\right)\right\}_{i=1}^{\infty}$ with $r_{\alpha_{i, \epsilon, n}}<\epsilon$ and $\alpha_{i, \epsilon, n} \subset \mathcal{A}$ for all $i \in \mathbb{N}$. In particular, we have a weak cover of $Y_{n}$ by disjoint balls chosen from the family $\left\{B\left(b_{\alpha_{i, \delta / t, n}}, r_{\alpha_{i, \delta / t, n}}\right)\right\}_{i=1}^{\infty}$ with all $r_{\alpha_{i, \delta / t, n}}<\delta / t$. Moreover, by definition, this weak cover has Lebesgue constant $B=1$. We may then apply Proposition [5.7 to the corresponding countable family of $\nu$-spikes.

Therefore,for any $\beta<1$ there exists $h_{n+1}(x) \in V_{+}$such that

$$
h_{n+1}(x) \leq \beta R_{n}(x) \quad \text { for all } \quad x \in X
$$

and

$$
\left\|h_{n+1}(x)\right\|_{L^{1}\left(Y_{n}\right)} \geq \frac{\beta L_{\nu}}{C_{n}^{2} s^{2}}\left\|R_{n}\right\|_{L^{1}\left(Y_{n}\right)} .
$$

Recall

$$
R_{n}(x)=F(x)-\sum_{i=1}^{n} h_{i}(x) \geq\left(1-\gamma_{n}\right) F(x),
$$

so it follows that

$$
F(x)-\sum_{i=1}^{n+1} h_{i}(x)=R_{n}(x)-h_{n+1}(x) \geq(1-\beta) R_{n}(x) \geq(1-\beta)\left(1-\gamma_{n}\right) F(x) .
$$

Therefore setting $\gamma_{n+1}=1-\left(1-\gamma_{n}\right)(1-\beta)<1$, we have

$$
\sum_{i=1}^{n+1} h_{i}(x) \leq \gamma_{n+1} F(x) .
$$


Using the above estimates we have,

$$
\begin{aligned}
\left\|R_{n+1}\right\|_{L^{1}(X)} & =\left\|R_{n}-h_{n+1}\right\|_{L^{1}(X)}=\left\|R_{n}\right\|_{L^{1}(X)}-\left\|h_{n+1}\right\|_{L^{1}(X)} \\
& \leq\left\|R_{n}\right\|_{L^{1}(X)}-\frac{L_{\nu}}{C_{n}^{2} s^{2}}\left\|R_{n}\right\|_{L^{1}\left(Y_{n}\right)} \\
& \leq\left(1-\frac{L_{\nu}}{C_{n}^{2} s^{2}}\right)\left\|R_{n}\right\|_{L^{1}(X)}+\frac{L_{\nu}}{C_{n}^{2} s^{2}}\|F\|_{L^{1}\left(X \backslash Y_{n}\right)}
\end{aligned}
$$

Recall $s$ was fixed independent of $n$. Moreover since $F \in L^{1}(X, \nu),\|F\|_{L^{1}\left(X \backslash Y_{n}\right)}$ tends to 0 as $n \rightarrow \infty$. Recall that $\frac{1}{T_{\nu}}$, and hence $L_{\nu}$, may tend to 0 as $C_{n}$ increases. However, we are free to choose how quickly $C_{n}$ tends to $\infty$. Therefore we choose the sequence $\left\{C_{n}\right\}_{n=1}^{\infty}$ tending to $\infty$ sufficiently slowly so that $\lim _{k \rightarrow \infty} \prod_{n=1}^{k}\left(1-\frac{L_{\nu}}{C_{n}^{2} s^{2}}\right)=0$. In other words, we force the sum $\sum_{n=1}^{\infty} \frac{L_{\nu}}{C_{n}^{2}}$ to diverge. The proof is finished by the next lemma, which we apply to the sequences $\delta_{n}=\frac{L_{\nu}}{C_{n}^{2} s^{2}}$ and $\epsilon_{n}=\|F\|_{L^{1}\left(X \backslash Y_{n}\right)}$.

Lemma 5.12. Let $0 \leq \delta_{n} \leq 1$ for all $n$ and $\lim _{n \rightarrow \infty} \epsilon_{n}=0$. Let $\left\{a_{n}\right\}_{n=1}^{\infty}$ be a sequence of non-negative numbers, such that

$$
a_{n+1} \leq\left(1-\delta_{n}\right) a_{n}+\delta_{n} \epsilon_{n} .
$$
Then

Denote by $\Delta_{m}^{n}=\prod_{k=m+1}^{n}\left(1-\delta_{k}\right)$, for $0 \leq m \leq n$ and $\Delta_{-1}^{n}=0$ and $\epsilon_{0}=a_{1}$.

$$
a_{n+1} \leq \sum_{k=0}^{n}\left(\Delta_{k}^{n}-\Delta_{k-1}^{n}\right) \epsilon_{k}
$$

In particular if $\lim _{n \rightarrow \infty} \epsilon_{n}=0$ and $\lim _{n \rightarrow \infty} \Delta_{k}^{n}=0$, for all $k \geq-1$ then $\lim _{n \rightarrow \infty} a_{n}=0$

Proof. Proof is by induction. For $n=0$ we have $a_{1} \leq\left(\Delta_{0}^{0}-\Delta_{-1}^{0}\right) \epsilon_{0}=a_{1}$. Assume we proved this for $k<n$. Now for $k=n$ we have

$$
a_{n+1} \leq\left(1-\delta_{n}\right) a_{n}+\delta_{n} \epsilon_{n} \leq\left(1-\delta_{n}\right) \sum_{k=0}^{n-1}\left(\Delta_{k}^{n-1}-\Delta_{k-1}^{n-1}\right) \epsilon_{k}+\left(1-\left(1-\delta_{n}\right)\right) \epsilon_{n} .
$$

Since $\left(1-\delta_{n}\right) \Delta_{k}^{n-1}=\Delta_{k}^{n}$ and $\Delta_{n}^{n}=1$ and $\Delta_{n-1}^{n}=1-\delta_{n}$, we have

$$
a_{n+1} \leq \sum_{k=0}^{n-1}\left(\Delta_{k}^{n}-\Delta_{k-1}^{n}\right) \epsilon_{k}+\left(\Delta_{n}^{n}-\Delta_{n-1}^{n}\right) \epsilon_{n}=\sum_{k=0}^{n}\left(\Delta_{k}^{n}-\Delta_{k-1}^{n}\right) \epsilon_{k} .
$$

This proves the formula.

Observe as $0 \leq \delta_{k} \leq 1$ we have that $\Delta_{k-1}^{n} \leq \Delta_{k}^{n}$, and $\Delta_{k}^{n+1} \leq \Delta_{k}^{n}$, for $0 \leq k \leq n$. For $\epsilon>0$ there exits $N$ such that for all $n>N, \epsilon_{n} \leq \epsilon$.

So

$a_{n} \leq \sum_{k=0}^{N-1}\left(\Delta_{k}^{n}-\Delta_{k-1}^{n}\right) \epsilon_{k}+\sum_{k=N}^{n}\left(\Delta_{k}^{n}-\Delta_{k-1}^{n}\right) \epsilon_{k} \leq \sum_{k=0}^{N-1}\left(\Delta_{k}^{n}-\Delta_{k-1}^{n}\right) \epsilon_{k}+\sum_{k=N}^{n}\left(\Delta_{k}^{n}-\Delta_{k-1}^{n}\right) \epsilon$

Since the second sum telescopes we obtain

$$
a_{n} \leq \sum_{k=0}^{N-1}\left(\Delta_{k}^{n}-\Delta_{k-1}^{n}\right) \epsilon_{k}+\left(\Delta_{n}^{n}-\Delta_{N-1}^{n}\right) \epsilon
$$


Sending $n \rightarrow \infty$ and using the fact that $\Delta_{n}^{n}=1$ and $\lim _{n \rightarrow \infty} \Delta_{k}^{n}=0$ for every $k \geq-1$, we obtain that $0 \leq \lim _{n \rightarrow \infty} a_{n} \leq \epsilon$. Sending, $\epsilon \rightarrow 0$, we complete the proof.

Observe that all of the families of spikes to which we applied Proposition 5.7 could have been chosen from the countable family corresponding to the indices $\left\{\alpha_{i, \frac{1}{j}, k}\right\}_{i, j, k=1}^{\infty} \subset \mathcal{A}$. This family is universal in that it does not depend on $F$, but only on a particular choice of Vitaly covers for the $B_{C_{n}}$. We henceforth re-index this family as $\left\{\alpha_{i}\right\}_{i=1}^{\infty} \subset \mathcal{A}$. The corresponding family of spikes is therefore the countable subfamily given by the theorem.

The second statement of the theorem follows by replacing the $L^{1}$ norm by absolute value and using the pointwise estimate given in part b) of the proposition. If $F$ is continuous the pointwise estimate gives uniform convergence on compact sets since the limit of partial sums is nondecreasing.

To prove the theorem for an $L^{1}$ almost lower semicontinuous function $F$, we first chose a nondecreasing sequence of continuous approximations $F_{1} \leq F_{2} \leq \cdots \leq F$. We may assume $F_{j}<F_{j+1}$ for all $j>0$ by replacing $F_{j}$ by $F_{j}-\epsilon_{j}$ for a sequence of sufficiently small $\epsilon_{j}>0$ which tend to 0 . Setting $F_{0}=0$ we may write

$$
F=\sum_{j=1}^{\infty} F_{j}-F_{j-1}
$$

Since $F_{j}-F_{j-1}$ is bounded, continuous and uniformly positive, it satisfies the hypotheses of the theorem and we have shown that $F_{j}-F_{j-1}=\sum_{i=1}^{\infty} \lambda_{\alpha_{i, j}} f_{\alpha_{i, j}}$. In particular,

$$
\left\|\sum_{j=1}^{\infty}\left(\sum_{i=1}^{\infty} \lambda_{\alpha_{i, j}} f_{\alpha_{i, j}}\right)\right\|_{1}=\left\|\sum_{j=1}^{\infty} F_{j}-F_{j-1}\right\|_{1}=\|F\|_{1}<\infty .
$$

However since the $f_{\alpha_{i, j}}$ and the $\lambda_{\alpha_{i, j}}$ are all positive, we are free to rearrange this sum. If we re-index the countable family $\left\{\alpha_{i, j}\right\}_{i, j=1}^{\infty}$ into our universal family of indices, $\left\{\alpha_{i}\right\}_{i=1}^{\infty}$, then we obtain

$$
0=\left\|F-\sum_{j=1}^{\infty}\left(\sum_{i=1}^{\infty} \lambda_{\alpha_{i, j}} f_{\alpha_{i, j}}\right)\right\|_{1}=\left\|F-\sum_{i=1}^{\infty} \lambda_{\alpha_{i}} f_{\alpha_{i}}\right\|_{1},
$$

where

$$
\lambda_{\alpha_{i}}= \begin{cases}0 & \left\{j: \alpha_{i}=\alpha_{i, j}\right\}=\emptyset \\ \sum_{\left\{j: \alpha_{i}=\alpha_{i, j}\right\}} \lambda_{\alpha_{i, j}} & \left\{j: \alpha_{i}=\alpha_{i, j}\right\} \neq \emptyset .\end{cases}
$$

The $\lambda_{\alpha_{i}}$ are the finite nonnegative coefficients guaranteed by the theorem.

Again the second statement of the theorem follows similarly by replacing the $L^{1}$ norm by the absolute value and working pointwise in the obvious way.

\section{EXISTENCE OF THE FIRST MOMENT FOR $Q$-SPIKES}

In this section we would like to strengthen the result of the Theorem 5.2 with some extra conditions. Recall that for lower semicontinuous functions we had $\sum_{i=1}^{\infty} \lambda_{i}\left\|f_{i}\right\|_{1}=\|F\|_{1}<\infty$. For our main applications we will need slightly better convergence properties. Namely, 
Theorem 6.1 (Finite Moment and Entropy Theorem). Assume that we are in the setting of Theorem 5.2 For convenience, assume the local doubling constants $T_{\nu, C}$ are bounded from above by $T_{\nu}<\infty$. In addition, assume that the family of unit $\nu$-spikes $\left\{\left(f_{\alpha}, r_{\alpha}, b_{\alpha}, Q, \theta, C_{\alpha}\right)\right\}_{\alpha \in \mathcal{A}}$ is a family of $Q$-spikes.

Assume that for every $C, \epsilon>0$ there is a finite Lebesgue subcover cover $\left\{B\left(b_{\alpha_{i}}, r_{\alpha_{i}}\right)\right\}_{i=1}^{N(C, \epsilon)}$ of $B_{C}$ such that $g(\epsilon) \leq r_{\alpha_{i}} \leq \epsilon$, for every $i \in \mathbb{N}$ and some positive increasing function $g:[0, \infty] \rightarrow[0, \infty]$ with $g(r)<r$ for $1>r>0$. For any uniformly positive bounded Lipschitz function $F$ with Lipschitz constant L, by Theorem 5.2 we may choose a positive sequence $\left\{\lambda_{\alpha_{i}}\right\}_{i=1}^{\infty}$ so that,

$$
F=\sum_{i=1}^{\infty} \lambda_{i} f_{i}(x)
$$

with uniform convergence. Moreover, if $g(\epsilon) \geq a \epsilon^{k}$ for some $0<a<1$ and $k \geq 1$, then there is a constant $A>0$, independent of $F$, such that the following hold.

1. If $k=1, \nu\left(B_{C}\right) \leq 1-\frac{1}{C^{6} \log ^{2}(C)}$, then we may choose the constants $\lambda_{i}>0$ so that in addition, the first moment satisfies

$$
\begin{aligned}
& \sum_{i=1}^{\infty} \lambda_{i}\left\|f_{i}\right\|_{L^{1}} \log \frac{1}{\left\|f_{i}\right\|_{L^{1}}}<A\left[1+\log \left(\frac{\sup _{z \in X} F(z)}{\inf _{z \in X} F(z)}\right)+\log L\right]\|F\|_{L^{\infty}}<\infty \\
& \quad \text { with entropy } \\
& -\sum_{i=1}^{\infty} \lambda_{i}\left\|f_{i}\right\|_{L^{1}} \log \left(\lambda_{i}\left\|f_{i}\right\|_{L^{1}}\right)<A\left[1+\log \left(\frac{\sup _{z \in X} F(z)}{\inf _{z \in X} F(z)}\right)+\log L\right]\|F\|_{L^{\infty}}<\infty .
\end{aligned}
$$

2. If $k=1$ and $\nu\left(B_{C}\right) \leq 1-\frac{1}{C^{2} \log ^{3}(C)}$, or $k>1$ and $\nu\left(B_{C}\right) \leq 1-\frac{1}{C^{4} \log ^{2}(C)}$, then we may choose the constants $\lambda_{i}>0$ so that in addition, the first log-moment satisfies

$$
\sum_{i=1}^{\infty} \lambda_{i}\left\|f_{i}\right\|_{L^{1}} \log \log \frac{1}{\left\|f_{i}\right\|_{L^{1}}}<A\left[1+\log \left(1+\log \left(\frac{\sup _{z \in X} F(z)}{\inf _{z \in X} F(z)}\right)+\log L\right)\right]\|F\|_{L^{\infty}}<\infty .
$$

3. if $\nu\left(B_{C}\right)=1$ for some fixed $C \geq 1$ and $\frac{C^{2}}{C^{2}-L_{\nu}}>k \geq 1$, then we could choose the constants $\lambda_{i} \geq 0$, such that the first moment satisfies

$$
\begin{aligned}
& \sum_{i=1}^{\infty} \lambda_{i}\left\|f_{i}\right\|_{L^{1}} \log \frac{1}{\left\|f_{i}\right\|_{L^{1}}}<A\left[1+\log \left(\frac{\sup _{z \in X} F(z)}{\inf _{z \in X} F(z)}\right)+\log L\right]\|F\|_{L^{1}}<\infty \\
& \quad \text { with entropy } \\
& -\sum_{i=1}^{\infty} \lambda_{i}\left\|f_{i}\right\|_{L^{1}} \log \left(\lambda_{i}\left\|f_{i}\right\|_{L^{1}}\right)<A\left[1+\log \left(\frac{\sup _{z \in X} F(z)}{\inf _{z \in X} F(z)}\right)+\log L\right]\|F\|_{L^{1}}<\infty .
\end{aligned}
$$

Remarks 6.2. In our applications we will need to use basis functions normalized to have unit mass instead of unit height, i.e. $f_{i} /\left\|f_{i}\right\|_{L^{1}}$ where $\left\|f_{i}\right\|_{L^{\infty}}=1$. In this case $\lambda_{i}\left\|f_{i}\right\|_{L^{1}}$ will be the coefficients of the sum instead of $\lambda_{i}$. Furthermore, in our applications to a group $\Gamma, \log \left(\frac{1}{\left\|f_{i}\right\|_{L^{1}}}\right)$ will roughly correspond to the word length $d_{\Gamma}\left(\mathbf{e}, \gamma_{i}\right)$ in $\Gamma$ and $\lambda_{i}\left\|f_{i}\right\|_{L^{1}}$ is the value of the measure $\mu\left(\gamma_{i}\right)$. With these 
substitutions, the first moment, first log-moment and entropy formulas stated in the above theorem respectively take on their more recognizable form of

$$
\sum_{\gamma \in \Gamma} \mu(\gamma) d_{\Gamma}(\mathbf{e}, \gamma), \quad \sum_{\gamma \in \Gamma} \mu(\gamma) \log d_{\Gamma}(\mathbf{e}, \gamma) \quad \text { and } \quad-\sum_{\gamma \in \Gamma} \mu(\gamma) \log \mu(\gamma)
$$

One may observe that the entropy formula is nearly that of the first moment, except for the presence of an additional $\lambda_{i}$ within the $\log$ term. For an $L^{\infty}$ function the $\lambda_{i}$ are bounded above, but not below. Therefore, the entropy estimate implies the first moment but not conversely. In particular, finite entropy need not hold in the weakened estimate of case 2). In Kai00 sufficient conditions for Poisson boundaries are established including finite log-moment together with various other possible estimates, not just finite entropy. It is for such potential usefulness that we include this case here.

The third case is of most interest to us, since this incorporates the setting of a (convex-)cocompact group of isometries of a $\operatorname{CAT}(-1)$ space acting on $X=\Lambda$ (see Section 8). However, this case still holds for any measure $\alpha$-conformal density $\nu$ on a $\delta$-hyperbolic space so long as the Busemann functions are Lipschitz. Also by taking $k>1$ more general groups than convex-cocompact ones may be considered.

Suppose we wish to weaken our assumption on $\nu$ by allowing the local doubling constants $T_{\nu, C}$ for the spikes with $C_{\alpha}<C$ to depend on $C$ so that they can become unbounded. Then the theorem still holds in cases 1) and 2) if we everywhere replace $C$ by $\sqrt{T_{\nu, C}} C$. For instance in 1),

$$
\nu\left(B_{C}\right) \leq 1-\frac{1}{C^{6} \log ^{2} C} \quad \text { becomes } \quad \nu\left(B_{C}\right) \leq 1-\frac{1}{T_{\nu, C}^{3} C^{6} \log ^{2}\left(\sqrt{T_{\nu, C}} C\right)} .
$$

However, in our main application of this theorem $T_{\nu}$ will be bounded above.

Proof of Theorem [6.1. We will examine the proof of Theorem 5.2 paying closer attention to $\delta$ and $t$. In case 3 ) we will assume for the moment that $F$ is Lipschitz and prove the general case only at the end. As shown in Theorem 5.2 we may restrict to a countable index subset of $\mathcal{A}$ whose corresponding family of spikes has the same properties. Therefore we will assume without loss of generality that $\mathcal{A}$ is countable.

Again the construction is by induction. By our assumption $T_{\nu}<\infty$, it follows that the constant $L_{\nu}<1$ from Proposition [5.7 is universally bounded from below. We will henceforth assume $L_{\nu}$ is this lower bound. Fix $s>2$ and recall that by the considerations in Theorem 5.2 we may assume $B_{C_{n}}$ is compact, otherwise we replace it by a compact set approximating it in measure. For case 1) we must take care to choose a sequence $C_{n}$ tending to infinity sufficiently slowly to obtain convergence in $L^{1}$, but no slower than necessary as there will be a trade-off with the speed of convergence of $\nu\left(B_{C_{n}}\right)$ to 1 . In case 1) choose the sequence $C_{n}$ to be $C_{n}=\max \left\{1, \sqrt{\frac{L_{\nu}}{5 s^{2}} n}\right\}$. It will turn out that this choice is roughly optimal.

Recall that $R_{N}(x)=F(x)-\sum_{n=1}^{N} h_{n}(x)$ where we build $h_{n}(x)$ by induction.

Assume that we have built $R_{N-1}(x)$. There exists $\delta_{N}>0$ such that for every $x \in X$ and $y \in B_{C_{N}}$ such that $d(x, y) \leq \delta_{N}$ we have

$$
s \geq \frac{R_{N-1}(y)}{R_{N-1}(x)} .
$$


Set $\epsilon_{0}=1$ and recursively define

$$
\epsilon_{i}=\min \left(\frac{\delta_{i}}{t_{i}}, \epsilon_{i-1}\right) .
$$

We may assume we have found $\epsilon_{N-1}$ and now we show that a sufficient choice of $\delta_{N}$ is

$$
\delta_{N}=\min \left\{\frac{(s-1) \inf _{x \in X} R_{N-1}(x)}{\sup _{y \in X} D_{g\left(\epsilon_{N-1}\right)}\left(R_{N-1}\right)(y)}, g\left(\epsilon_{N-1}\right)\right\} .
$$

Indeed, if $x \in X$ and $y \in B_{C_{N}}$ are such that $d(x, y) \leq \delta_{N} \leq g\left(\epsilon_{N-1}\right)$ then

$$
\begin{aligned}
s & \geq \frac{\delta_{N} \sup _{z \in B_{C_{N}}} D_{g\left(\epsilon_{N-1}\right)} R_{N-1}(z)}{\inf _{z \in X}\left(R_{N-1}(z)\right)}+1 \\
& \geq \frac{\delta_{N}}{\inf _{z \in X}\left(R_{N-1}(z)\right)} \sup _{\substack{z \in B_{C_{N}} \\
0<d(x, z) \leq g\left(\epsilon_{N-1}\right)}} \frac{\left|R_{N-1}(z)-R_{N-1}(x)\right|}{d(x, z)}+1 \\
& \geq \frac{R_{N-1}(y)-R_{N-1}(x)}{R_{N-1}(x)}+1=\frac{R_{N-1}(y)}{R_{N-1}(x)} .
\end{aligned}
$$

Also recall that

$$
t_{N}=\left(\frac{\sup _{y \in B_{C_{N}}}\left(R_{N-1}(y)\right)}{\inf _{x \in X}\left(R_{N-1}(x)\right)}\right)^{\frac{1}{Q}}+1 .
$$

Since we can choose a finite Besecovitch cover $\left\{B\left(b_{\alpha_{i}^{(N)}}, r_{\alpha_{i}^{(N)}}\right)\right\}_{i=1}^{k_{N}}$ with $g\left(\epsilon_{N}\right) \leq$ $r_{\alpha_{i}^{(N)}} \leq \epsilon_{N}$, we can apply Proposition [5.7 b) to the function $\beta R_{N-1}$ for a fixed constant $\beta<1$. Specifically, there exists a $h_{N}(x)=\sum_{i=1}^{k_{N}} \lambda_{i}^{(N)} f_{\alpha_{i}^{(N)}}(x) \in V_{+}$such that we have

$$
h_{N}(x) \leq \beta R_{N-1}(x),
$$

and

$$
\frac{L_{\nu} \beta}{C_{N}^{2} s^{2}}\left\|R_{N-1}(x)\right\|_{B_{C_{N}}} \leq\left\|h_{N}\right\|_{B_{C_{N}}} .
$$

As will become apparent, the role of the constant $\beta$ is simply to make certain that $R_{N-1}-h_{N}$ is uniformly positive. Recall that for all $N>\frac{5 s^{2}}{L_{\nu}}$, we have $C_{N}=\sqrt{\frac{L_{\nu}}{5 s^{2}} N}$. From now on we fix $s \in(1,2)$ and without loss of generality assume $\beta$ was chosen sufficiently close to 1 so that for all $N>\frac{5 s^{2}}{L_{\nu}}$,

$$
\frac{5}{N} \geq \frac{L_{\nu} \beta}{C_{N}^{2} s^{2}} \geq \frac{4}{N} .
$$

(In the case 3 ) where $\nu\left(B_{C}\right)=1$ and $\frac{1}{k}+\frac{L_{\nu}}{C^{2}}>1$ chose $s$ and $\beta$ sufficiently close to 1 so that $\frac{1}{k}+\frac{L_{\nu} \beta}{s^{2} C^{2}}>1$.)

Therefore we obtain

$$
R_{N}(x)=R_{N-1}(x)-h_{N}(x) \geq(1-\beta) R_{N-1}(x),
$$

or inductively,

$$
R_{N}(x) \geq F(x)(1-\beta)^{N} .
$$


Setting $Y_{n}=B_{C_{N}}$ from inequality (5.1) the fact that $\left\|R_{N-1}\right\|_{L^{\infty}(X)} \leq\|F\|_{L^{\infty}(X)}$, we have

$$
\left\|R_{N}\right\|_{L^{1}(X)} \leq\left(1-\frac{L_{\nu} \beta}{C_{N}^{2} s^{2}}\right)\left\|R_{N-1}\right\|_{L^{1}(X)}+\frac{L_{\nu} \beta}{C_{N}^{2} s^{2}}\|F\|_{L^{\infty}(X)} \nu\left(X-B_{C_{N}}\right) .
$$

By Lemma $5.12 R_{N} \rightarrow 0$ since $\sum_{i=1}^{\infty} \frac{1}{C_{i}^{2}}=\infty$ and $\nu\left(B_{C_{N}}\right) \rightarrow 1$. Recall that for each $a>0, \lim _{N \rightarrow \infty} N^{a} \prod_{i=[a]+1}^{N}\left(1-\frac{a}{i}\right)$ is a positive number. We may solve the recursive inequality (6.1) for $\left\|R_{N}\right\|_{L^{1}(X)}$ given above (see Lemma 5.12). For some constants $C^{\prime}$ and $C^{\prime \prime}$ we obtain

$$
\begin{aligned}
\left\|R_{N+1}\right\|_{L^{1}(X)} & \leq\|F\|_{L^{\infty}(X)} \sum_{k=1}^{N} \frac{L_{\nu} \beta \nu\left(X-B_{C_{k}}\right)}{C_{k}^{2} s^{2}}\left[\prod_{i=k+1}^{N}\left(1-\frac{L_{\nu} \beta}{C_{i}^{2} s^{2}}\right)\right] \\
& \leq\|F\|_{L^{\infty}(X)} \sum_{k=1}^{N} \frac{C^{\prime \prime} \nu\left(X-B_{C_{k}}\right)}{k}\left[\prod_{i=k+1}^{N}\left(1-\frac{4}{i}\right)\right] \\
& \leq\|F\|_{L^{\infty}(X)} \sum_{k=1}^{N} \frac{C^{\prime} \nu\left(X-B_{C_{k}}\right)}{k}\left(\frac{k}{N}\right)^{4} .
\end{aligned}
$$

Therefore in case 1), the sum becomes $\|F\|_{L^{\infty}(X)} N^{-4} \sum_{k=1}^{N} \frac{C^{\prime}}{\log ^{2}(k)}$. It is straightforward to bound this sum from above we bound this sum from above, say by by integration. After relabelling $C^{\prime}$ we may obtain a bound of

$$
\left\|R_{N+1}\right\|_{L^{1}(X)} \leq \frac{C^{\prime}\|F\|_{L^{\infty}(X)}}{N^{3} \log ^{2}(N)} .
$$

In case 2), where in either case $\nu\left(B_{C}\right) \leq\left(1-\frac{k}{\log (k) C^{4} \log ^{2}(C)+C^{2} \log ^{3}(C)}\right)$, the same estimate yields

$$
\left\|R_{N+1}\right\|_{L^{1}(X)} \leq \frac{C^{\prime}\|F\|_{L^{\infty}(X)}}{\log (k) N^{2} \log ^{2}(N)+N \log ^{3}(N)} .
$$

In the case 3$)$, where $\nu\left(B_{C}\right)=1$, we have

$$
\left\|R_{N+1}\right\|_{L^{1}} \leq\|F\|_{L^{1}}\left(1-\frac{L_{\nu} \beta}{C^{2} s^{2}}\right)^{N}
$$

Therefore, we obtain that

$$
F(x)=\sum_{N=1}^{\infty} h_{N}(x)=\sum_{N=1}^{\infty} \sum_{i=1}^{k_{N}} \lambda_{i}^{(N)} f_{\alpha_{i}^{(N)}}(x)
$$

with convergence in $L^{1}$-norm. Also

$$
\sum_{i=1}^{k_{N}} \lambda_{i}^{(N)}\left\|f_{\alpha_{i}^{(N)}}\right\|_{L^{1}}=\left\|h_{N}\right\|_{L^{1}} \leq \beta\left\|R_{N-1}\right\|_{L^{1}} .
$$


Therefore, after renaming $C^{\prime}$ we have

$$
\sum_{i=1}^{k_{N}} \lambda_{i}^{(N)}\left\|f_{\alpha_{i}^{(N)}}\right\|_{L^{1}(X)} \leq \begin{cases}\frac{C^{\prime}\|F\|_{L^{\infty}(X)}}{N^{3} \log ^{2}(N)} & \text { case 1) } \\ \frac{C^{\prime}\|F\|_{L^{\infty}(X)}}{\log (k) N^{2} \log ^{2}(N)+N \log ^{3}(N)} & \text { case 2) } \\ C^{\prime}\|F\|_{L^{1}(X)}\left(1-\frac{L_{\nu} \beta}{C^{2} s^{2}}\right) & \text { case 3) }\end{cases}
$$

Now we would like to connect $\epsilon_{N+1}$ to $\epsilon_{N}$. (it might seem that it is easier to connect $\epsilon_{N}$ and $\epsilon_{N-1}$, but the former relation is easier to write).

Since $h_{N}(x) \in V_{+}$and $r_{\alpha_{i}^{(N)}} \geq g\left(\epsilon_{N}\right)$ and $h_{N}(x)$ is composed of $Q$-spikes we have,

$$
D_{g\left(\epsilon_{N}\right)} R_{N}(x) \leq D_{g\left(\epsilon_{N}\right)} R_{N-1}(x)+D_{g\left(\epsilon_{N}\right)} h_{N}(x)
$$

So

$$
\begin{aligned}
D_{g\left(\epsilon_{N}\right)} h_{N}(x) & \leq \sum_{i=1}^{k_{N}} \lambda_{i}^{(N)} D_{g\left(\epsilon_{N}\right)} f_{\alpha_{i}^{(N)}}(x) \leq \sum_{i=1}^{k_{N}} \lambda_{i}^{(N)} D_{r_{\alpha_{i}^{(N)}}} f_{\alpha_{i}^{(N)}}(x) \\
& \leq \sum_{i=1}^{k_{N}} \lambda_{i}^{(N)} \frac{C_{N} f_{\alpha_{i}^{(N)}}(x)}{r_{\alpha_{i}^{(N)}}^{(N)}} \leq \frac{C_{N}}{g\left(\epsilon_{N}\right)} h_{N}(x) \\
& \leq \frac{C_{N}}{g\left(\epsilon_{N}\right)} \beta R_{N-1}(x) .
\end{aligned}
$$

In particular, since $g\left(\epsilon_{N}\right) \leq g\left(\epsilon_{N-1}\right)$,

$$
\begin{aligned}
\sup _{z \in X}\left(D_{g\left(\epsilon_{N}\right)} R_{N}(z)\right) & \leq \sup _{z \in X}\left(D_{g\left(\epsilon_{N}\right)} R_{N-1}(z)\right)+\sup _{z \in X} D_{g\left(\epsilon_{N}\right)} h_{N}(z) \\
& \leq \sup _{z \in X}\left(D_{g\left(\epsilon_{N-1}\right)} R_{N-1}(z)\right)+\sup _{z \in X} D_{g\left(\epsilon_{N}\right)} h_{N}(z),
\end{aligned}
$$

and recalling that $g\left(\epsilon_{N}\right) \leq \epsilon_{N} \leq \frac{\delta_{N}}{t_{N}}$ and

$$
\sup _{z \in X}\left(D_{g\left(\epsilon_{N-1}\right)} R_{N-1}(z)\right) \leq \frac{(s-1) \inf _{z \in X}\left(R_{N-1}(z)\right)}{\delta_{N}} \leq \frac{(s-1) \inf _{z \in X}\left(R_{N-1}(z)\right)}{t_{N} \epsilon_{N}}
$$

the previous inequality becomes, for $N \geq 1$,

$$
\begin{aligned}
\sup _{z \in X}\left(D_{g\left(\epsilon_{N}\right)} R_{N}(z)\right) & \leq \frac{(s-1) \inf _{z \in X}\left(R_{N-1}(z)\right)}{t_{N} \epsilon_{N}}+\frac{C_{N} \beta}{g\left(\epsilon_{N}\right)} \sup _{z \in X}\left(R_{N-1}(z)\right) \\
& \leq\left(\frac{s-1}{t_{N}}+C_{N} \beta\right) \frac{\sup _{z \in X}\left(R_{N-1}(z)\right)}{g\left(\epsilon_{N}\right)} \\
& \leq(s-1) C_{N} \frac{\sup _{z \in X}\left(R_{N-1}(z)\right)}{g\left(\epsilon_{N}\right)}
\end{aligned}
$$

since $t_{N} \geq 2, \beta<1$ and $s>2$.

Therefore using the above estimate, we have

$$
\delta_{N+1}=\min \left\{\frac{(s-1) \inf _{z \in X}\left(R_{N}(z)\right)}{\sup _{z \in X}\left(D_{g\left(\epsilon_{N}\right)} R_{N}(z)\right)}, g\left(\epsilon_{N}\right)\right\} \geq \frac{\inf _{z \in X}\left(R_{N}(z)\right)}{C_{N} \sup _{z \in X}\left(R_{N-1}(z)\right)} g\left(\epsilon_{N}\right)
$$

Therefore we obtain,

$$
\frac{\delta_{N+1}}{t_{N+1}} \geq \frac{\inf _{z \in X}\left(R_{N}(z)\right)}{C_{N} \sup _{z \in X}\left(R_{N-1}(z)\right) t_{N+1}} g\left(\epsilon_{N}\right) .
$$


Now we have

$$
t_{N+1}-1=\left(\frac{\sup _{z \in B_{C_{N+1}}}\left(R_{N}(z)\right)}{\inf _{z \in X}\left(R_{N}(z)\right)}\right)^{\frac{1}{Q}} \leq\left(\frac{1}{(1-\beta)^{N}} \frac{\sup _{z \in X} F(z)}{\inf _{z \in X} F(z)}\right)^{\frac{1}{Q}},
$$

and since $t_{N} \geq 2$ we also have

$$
t_{N} \leq 2\left(t_{N}-1\right)
$$

Therefore we obtain

$$
\frac{\delta_{N+1}}{t_{N+1}} \geq \frac{(1-\beta)^{N\left(1+\frac{1}{Q}\right)}}{2(1-\beta) C_{N}} g\left(\epsilon_{N}\right)\left(\frac{\inf _{z \in X} F(z)}{\sup _{z \in X} F(z)}\right)^{N\left(1+\frac{1}{Q}\right)} .
$$

Since $\epsilon_{N}>(1-\beta)^{N\left(1+\frac{1}{Q}\right)} g\left(\epsilon_{N}\right)$, we have

$$
\begin{aligned}
\epsilon_{N+1}=\min \left(\frac{\delta_{N+1}}{t_{N+1}}, \epsilon_{N}\right) & \geq \frac{(1-\beta)^{N\left(1+\frac{1}{Q}\right)}}{2 C_{N}} g\left(\epsilon_{N}\right)\left(\frac{\inf _{z \in X} F(z)}{\sup _{z \in X} F(z)}\right)^{\left(1+\frac{1}{Q}\right)} \\
& \geq K^{\prime}\left((1-\beta)^{N} \frac{\inf _{z \in X} F(z)}{\sup _{z \in X} F(z)}\right)^{2} g\left(\epsilon_{N}\right),
\end{aligned}
$$

for some constant $K^{\prime}<1$ which is independent of $N$ since $C_{N}$ is dominated by the additional exponential term. This is still true in case 3) of the theorem where we may consider $C_{N}$ to be eventually constant.

Lemma 6.3. Assume $g(\epsilon) \geq a \epsilon^{k}$ for some $1>a>0$ and $k \geq 1$.

(1) if $k=1$, then there exists $\lambda_{0}>0$, independent of $F$, such that for all $N$ we have

$$
\epsilon_{N+1} \geq \frac{e^{-\lambda N^{2}}}{\sup _{z \in X} D_{1} F(z)} \quad \text { where } \quad \lambda=\lambda_{0}\left[1+\log \left(\frac{\sup _{z \in X} F(z)}{\inf _{z \in X} F(z)}\right)\right] .
$$

(2) if $k>1$, then there exists $\lambda_{0}>0$, independent of $F$, such that for all $N$ we have

$$
\epsilon_{N+1} \geq e^{-\lambda k^{N}} \quad \text { where } \quad \lambda=\lambda_{0}\left[1+\log \left(\frac{\sup _{z \in X} F(z)}{\inf _{z \in X} F(z)}\right)+\log \left(\sup _{z \in X} D_{1} F(z)\right)\right] .
$$

Proof. Let $a_{N}=\log \left(\epsilon_{N}\right)$. Making a gross underestimate, the inequality

$$
\epsilon_{N+1} \geq K^{\prime}\left((1-\beta) \frac{\inf _{z \in X} F(z)}{\sup _{z \in X} F(z)}\right)^{2 N} g\left(\epsilon_{N}\right)
$$

implies for $N \geq 1$,

$$
a_{N+1} \geq 2 N \log \left((1-\beta) \frac{\inf _{z \in X} F(z)}{\sup _{z \in X} F(z)}\right)+k a_{N}+\log \left(K^{\prime} a\right) .
$$

So inductively we obtain

$$
a_{N+1} \geq 2 \log \left((1-\beta) \frac{\inf _{z \in X} F(z)}{\sup _{z \in X} F(z)}\right) \sum_{i=1}^{N}(N-i) k^{i}+k^{N} a_{1}+\log \left(K^{\prime} a\right) \sum_{i=1}^{N} k^{i} .
$$


Recall that $R_{0}=F$ so $\epsilon_{1}=\frac{\delta_{1}}{t_{1}}>\frac{\left(\frac{\inf _{z \in X} F(z)}{2 \sup _{z \in X} F(z)}\right)^{2}}{\sup _{z \in X} D_{1} F(z)}$. If $k=1$, then since $1-\beta<1$ and $K^{\prime} a<1$ we have that

$$
\begin{aligned}
a_{N+1} & \geq 2 \log \left((1-\beta) \frac{\inf _{z \in X} F(z)}{\sup _{z \in X} F(z)}\right) \sum_{i=1}^{N}(N-i)+a_{1}+N \log \left(K^{\prime} a\right) \\
& \geq 2 \log \left((1-\beta) \frac{\inf _{z \in X} F(z)}{\sup _{z \in X} F(z)}\right) \frac{N^{2}+N}{2}+a_{1}+N \log \left(K^{\prime} a\right) \\
& \geq-\lambda_{0}\left[1+\log \left(\frac{\sup _{z \in X} F(z)}{\inf _{z \in X} F(z)}\right)\right] N^{2}-\log \sup _{z \in X} D_{1} F(z)
\end{aligned}
$$

for some $\lambda_{0}>0$. This proves 1$)$.

If $k>1$, then

$$
\begin{aligned}
a_{N+1} & \geq 2 \log \left((1-\beta) \frac{\inf _{z \in X} F(z)}{\sup _{z \in X} F(z)}\right) \sum_{i=0}^{N}(N-i) k^{i}+k^{N} a_{1}+\log \left(K^{\prime} a\right) \sum_{i=0}^{N} k^{i} \\
& =k^{N}\left(2 \log \left((1-\beta) \frac{\inf _{z \in X} F(z)}{\sup _{z \in X} F(z)}\right) \sum_{i=0}^{N} i k^{-i}+a_{1}+\log \left(K^{\prime} a\right) \sum_{i=0}^{N} k^{i-N}\right) \\
& \geq-\lambda_{0}\left[1+\log \left(\frac{\sup _{z \in X} F(z)}{\inf _{z \in X} F(z)}\right)+\log \left(\sup _{z \in X} D_{1} F(z)\right)\right] k^{N},
\end{aligned}
$$

for some $\lambda_{0}>0$.

This proves the lemma.

Now we continue with the proof of Theorem 6.1 By overestimating the first case, we can incorporate both cases of Lemma 6.3 into one estimate. Namely, there exists a $\lambda>0$ given by the second part of the lemma such that

$$
\epsilon_{N+1} \geq e^{-\lambda N^{2} k^{N}}
$$

Now from the definition of a spike, we have that for all $N$ and $i=1, \ldots, k_{N}$, if $K=\frac{L_{\nu} \beta}{5 s^{2}}$, then

$$
\frac{\left\|f_{\alpha_{i}^{(N)}}\right\|_{L^{1}}}{\left\|f_{\alpha_{i}^{(N)}}\right\|_{L^{\infty}}} \geq \frac{\nu\left(B\left(b_{\alpha_{i}^{(N)}}, r_{\alpha_{i}^{(N)}}\right)\right)}{C_{N}} \geq \frac{r_{i}^{Q}}{C_{N}^{2}} \geq \frac{e^{-\lambda Q(N-1)^{2} k^{(N-1)}}}{K N}
$$

In case 3 ), this becomes

$$
\frac{\left\|f_{\alpha_{i}^{(N)}}\right\|_{L^{1}}}{\left\|f_{\alpha_{i}^{(N)}}\right\|_{L^{\infty}}} \geq \frac{e^{-\lambda Q(N-1)^{2} k^{(N-1)}}}{C^{2}}
$$

Now we use the estimate (**) and the above estimates to finish each case. We set the constant $L$ to be the global Lipschitz constant for $F$. 
Case 1). Using the fact that spikes are unit, our construction yields

$$
\begin{aligned}
\sum_{N=1}^{\infty} \sum_{i=1}^{k_{N}} & \lambda_{i}^{(N)}\left\|f_{\alpha_{i}^{(N)}}\right\|_{L^{1}} \log \left(\frac{1}{\left\|f_{\alpha_{i}^{(N)}}\right\|_{L^{1}}}\right) \\
& \leq \sum_{N=1}^{\infty} \sum_{i=1}^{k_{N}} \lambda_{i}^{(n)}\left\|f_{\alpha_{i}^{(N)}}\right\|_{L^{1}}\left(\lambda Q(N-1)^{2}+2 \log (K N)\right) \\
& \leq \sum_{N=1}^{\infty} \frac{C^{\prime}\|F\|_{L^{\infty}(X)}}{N^{3} \log ^{2}(N)}\left(\lambda Q(N-1)^{2}+2 \log (K N)\right) \\
& <A\left[1+\log \left(\frac{\sup _{z \in X} F(z)}{\inf _{z \in X} F(z)}\right)+\log L\right]\|F\|_{L^{\infty}(X)}<\infty
\end{aligned}
$$

Case 2). Using that $\log (x+y) \leq \log (x)+y$ for $x \geq 1$ and $y>0$, for a possibly different constant $C^{\prime}$ we obtain,

$$
\begin{aligned}
& \sum_{N=1}^{\infty} \sum_{i=1}^{k_{N}} \lambda_{i}^{(N)}\left\|f_{\alpha_{i}^{(N)}}\right\|_{L^{1}} \log \log \left(\frac{1}{\left\|f_{\alpha_{i}^{(N)}}\right\|_{L^{1}}}\right) \\
& \leq \sum_{N=1}^{\infty} \sum_{i=1}^{k_{N}} \lambda_{i}^{(n)}\left\|f_{\alpha_{i}^{(N)}}\right\|_{L^{1}} \log \left(\lambda Q(N-1)^{2} k^{N-1}+2 \log (K N)\right) \\
& \leq \sum_{N=1}^{\infty} \frac{C^{\prime}\|F\|_{L^{\infty}(X)}}{\log (k) N^{2} \log ^{2}(N)+N \log ^{3}(N)}(N \log (k)+\log (\lambda N)) \\
& <A\left[1+\log \left(1+\log \left(\frac{\sup _{z \in X} F(z)}{\inf _{z \in X} F(z)}\right)+\log L\right)\right]\|F\|_{L^{\infty}(X)}<\infty .
\end{aligned}
$$

Case 3). The construction yields

$$
\begin{aligned}
\sum_{N=1}^{\infty} \sum_{i=1}^{k_{N}} \lambda_{i}^{(N)}\left\|f_{\alpha_{i}^{(N)}}\right\|_{L^{1}} \log \left(\frac{1}{\left\|f_{\alpha_{i}^{(N)}}\right\|_{L^{1}}}\right) \\
\leq \sum_{N=1}^{\infty} \sum_{i=1}^{k_{N}} \lambda_{i}^{(n)}\left\|f_{\alpha_{i}^{(N)}}\right\|_{L^{1}}\left(\lambda Q(N-1)^{2} k^{N-1}+2 \log (C)\right) \\
\leq \sum_{N=1}^{\infty} C^{\prime}\|F\|_{L^{1}}\left(1-\frac{L_{\nu} \beta}{C^{2} s^{2}}\right)^{N}\left(\lambda Q(N-1)^{2} k^{N-1}+2 \log (C)\right) \\
<A\left[1+\log \left(\frac{\sup _{z \in X} F(z)}{\inf _{z \in X} F(z)}\right)+\log L\right]\|F\|_{L^{1}}<\infty .
\end{aligned}
$$

Since $\frac{1}{k}+\frac{L_{\nu} \beta}{C^{2} s^{2}}>1$ we have that

$$
\left(1-\frac{L_{\nu} \beta}{C^{2} s^{2}}\right) k<1
$$

and in this case the sum converges. Note that in each case the constant $A$ does not depend on $F$.

For the bound on the entropy we recall that $k_{N}$ is the number of elements in the Besicovitch cover with radii on the scale of $\epsilon_{N}$. By passing to a subcover, we may 
assume $k_{N}$ has been chosen minimally. Since the cover is Besicovitch and by the condition on $\nu$ in the definition of a $Q$-spike, we have

$$
k_{N} \leq \frac{K}{\epsilon_{N}^{Q}} \leq K L e^{\lambda Q(N-1)^{2} k^{N-1}}
$$

for some constant $K$ independent of $N$.

Now as before we break the computation into cases. The convexity of $-\log$ implies for case 1),

$$
\begin{aligned}
-\sum_{N=1}^{\infty} \sum_{i=1}^{k_{N}} \lambda_{i}^{(N)}\left\|f_{\alpha_{i}^{(N)}}\right\|_{L^{1}} \log \left(\lambda_{i}^{(N)}\left\|f_{\alpha_{i}^{(N)}}\right\|_{L^{1}}\right) \\
\leq-\sum_{N=1}^{\infty}\left(\sum_{i=1}^{k_{N}} \lambda_{i}^{(N)}\left\|f_{\alpha_{i}^{(N)}}\right\|_{L^{1}}\right) \log \left(\frac{1}{k_{N}} \sum_{i=1}^{k_{N}} \lambda_{i}^{(N)}\left\|f_{\alpha_{i}^{(N)}}\right\|_{L^{1}}\right) \\
\leq-\sum_{N=1}^{\infty} \frac{C^{\prime}\|F\|_{L^{\infty}(X)}}{N^{3} \log ^{2}(N)} \log \left(\frac{C^{\prime}\|F\|_{L^{\infty}(X)}}{k_{N} N^{3} \log ^{2}(N)}\right) \\
\leq \sum_{N=1}^{\infty} \frac{C^{\prime \prime}\|F\|_{L^{\infty}(X)}}{N^{3} \log ^{2}(N)}\left(\lambda Q N^{2}+4 \log (N)-\log \|F\|_{L^{\infty}(X)}\right) \\
<A\left[1+\log \left(\frac{\sup _{z \in X} F(z)}{\inf _{z \in X} F(z)}\right)+\log L\right]\|F\|_{L^{\infty}(X)}<\infty .
\end{aligned}
$$

Repeating this procedure for case 3) using the estimate in $\mathbb{N}^{*}$ is similar. This completes the proof of the theorem.

Remarks 6.4. If we wish to extend the theorem to a more general class of $F$, then we can approximate a bounded uniformly positive lower semicontinuous $F \in L^{1}(X, \nu)$ by an increasing sequence of Lipschitz (see [Mic01]) functions and use an argument similar to that in the proof of Theorem 5.2 Because the estimates are in terms of $\|F\|_{L^{\infty}}$ in case 1) and 2), this method fails. In fact, it is easy to construct a continuous function $F$ on the interval $[0,1]$ for which any increasing sequence of Lipschitz approximations $F_{j}$ satisfies $\sum_{j=1}^{\infty}\left\|F_{j}-F_{j-1}\right\|_{\infty}=\infty$.

However, in case 3) this approach can work. One explicit way of doing this is to consider the metric space version of the quadratic Moreau-Yosida infimal convolution approximation. Namely, let $F_{s}$ be defined by

$$
F_{s}(x):=\inf _{y \in X}\left\{F(y)+s d(x, y)^{2}\right\} .
$$

For lower semicontinuous $F$, the functions $F_{s}$ satisfy

(1) For all $s \geq 0, F_{s}$ is Lipschitz with Lipschitz constant $s$.

(2) For all $t \geq s \geq 0$, we have $F_{s} \leq F_{t} \leq F$ and $\lim _{s \rightarrow \infty} F_{s}(x)=F(x)$.

(3) For all $s \geq 0$, we have $\inf _{x \in X} F_{s}(x)=\inf _{x \in X} F(x)=H_{0}$.

Items 2) and 3) are routine to verify. For the first statement, see Chapter 5 of CLSW98 or CLW95 and observe that the proof only depends on the triangle inequality and not on the distance being a norm.

If $F$ arises as a strictly increasing pointwise limit of such uniformly positive bounded Lipschitz functions $F_{i}$ with Lipschitz constants $L_{i}$ and

$$
\sum_{i=1}^{\infty} \log \left(L_{i}\right)\left\|F_{i}-F_{i-1}\right\|<\infty
$$


then it is not hard to show that the conclusion of case 3) of the theorem still holds, except for the quantitative bounds. However this condition most likely fails for continuous functions whose pointwise modulus of continuity is greater than $-\frac{1}{\log }$ on a positive measure set, but we did not check this.

\section{RADON-NiKOdYM DERIVATIVES ARE SPIKES FOR $\delta$-HYPERBOLIC SPACES.}

Now we recall the notation of the first section. For a Gromov $\delta$-hyperbolic space $(X, d)$ and $\alpha>0$, we would like to find an $\alpha$-quasiconformal measure, but we do not know that any exist. If we let $\frac{\alpha}{\epsilon}$ be the Hausdorff dimension of the metric $\delta_{p}^{\epsilon}$ (or equivalently, the bilipschitz quasimetric $d_{p}^{\epsilon}$ ) and let $m_{p}$ be the corresponding $\frac{\alpha}{\epsilon}$-dimensional Hausdorff measure, then $m_{p}$ is a reasonable candidate for such a measure. In the case that $X$ is geodesic and $\Gamma$ acts quasiconvex cocompactly on $X$, Coornaert showed (Proposition 7.5 of Coo93), based on an argument of Sullivan Sul79, that $m_{p}$ is $\alpha$-quasiconformal and any two $\alpha$-quasiconformal measures are equivalent with a bounded uniformly positive Radon-Nikodym derivative. Nevertheless, even in this setting, it is not clear that there exist continuous $\alpha$ quasiconformal measures.

In this section we will prove Theorem 0.1 For this purpose we now allow $\alpha$ to be any positive number. However, we have already seen in Sections 1 and 2 that, at least for the somewhat regular spaces, we must assume $\alpha$ to be sufficiently large in order for $\alpha$-quasiconformal measures even to exist.

Proposition 7.1. Let $X$ be a $\delta$-hyperbolic metric space which is Gromov product bounded with respect to $\Gamma$ and some $p \in X$. For any $\alpha>0$, let $\nu$ be an $\alpha$-quasiconformal measure which does not consist of a single atom. Then there exist constants $\beta \geq 1$ and $D_{0}>0$, independent of $p$, such that for all $D \geq D_{0}$, all $\epsilon>0$ and all $\gamma \in \Gamma$,

$$
\left(e^{2 \alpha\left(x \cdot \gamma^{-1} p\right)_{p}}, e^{-\epsilon\left(U_{p, \gamma}-D\right)}, z_{p, \gamma}^{+}, \frac{\alpha}{\epsilon}, \frac{\alpha}{\epsilon}, \beta e^{\alpha(10 \delta+2 D)}\right)
$$

is a $\nu$-spike on $\partial X$ with respect to the quasimetric $d_{p}^{\epsilon}$.

Proof. We must verify the conditions of Definition 4.1 Recall that

$$
O_{p}(\gamma, D)=\left\{x: d_{p}^{\epsilon}\left(z_{p, \gamma}^{+}, x\right) \leq e^{-\epsilon\left(U_{p, \gamma}-D\right)}\right\} .
$$

By the Shadow Lemma (1.26) there exist $D_{0}$ and $\beta \geq 1$ such that for all $D \geq D_{0}$ we have

$$
\nu\left(O_{p}(\gamma, D)\right) \leq \beta e^{-\alpha U_{p, \gamma}} e^{2 \alpha D} .
$$

We also have

$$
\frac{1}{\beta} e^{-\alpha U_{p, \gamma}} \leq \nu\left(O_{p}(\gamma, D)\right) .
$$

Now for all $z, x \in \partial X$ such that $d_{p}^{\epsilon}(z, x) \leq e^{\epsilon\left(U_{p, \gamma}-D\right)}$ by Lemma 1.12 observe that $\left|\left(x \cdot \gamma^{-1} p\right)_{p}-\left(z \cdot \gamma^{-1} p\right)_{p}\right| \leq 2 \delta+D$. In particular

$$
e^{2 \alpha\left(x \cdot \gamma^{-1} p\right)_{p}} \geq e^{-2 \alpha(2 \delta+D)} e^{2 \alpha\left(z \cdot \gamma^{-1} p\right)_{p}} .
$$

This implies condition 3). 
Now it is not difficult to obtain condition 1). For all $x \in \partial X$ such that $d_{p}^{\epsilon}\left(z_{p, \gamma}^{+} x\right) \leq e^{\epsilon\left(U_{p, \gamma}-D\right)}$, we also have that

$$
e^{2 \alpha\left(x \cdot \gamma^{-1} p\right)_{p}} \geq e^{-2 \alpha(2 \delta+D)} e^{2 \alpha\left(z_{p, \gamma}^{+} \cdot \gamma^{-1} p\right)_{p}} \geq e^{-2 \alpha(4 \delta+D)} e^{2 \alpha U_{p, \gamma},}
$$

since $\left(z_{p, \gamma}^{+} \cdot \gamma^{-1} p\right)_{p} \geq U_{p, \gamma}-\delta$. This implies condition 1$)$.

Condition 2): By definition of $U_{p, \gamma}$ it follows from the Shadow Lemma 1.26 that $\nu$ has $\left(\frac{\alpha}{\epsilon}, \frac{\alpha}{\epsilon}\right)$-decay. For this case we also assume $x \notin O_{p}(\gamma, D)$, so we have $\left(x \cdot z_{p, \gamma}^{+}\right)_{p} \leq U_{p, \gamma}-D$. On the other hand, for all $y \in O_{p}(\gamma, D)$ we have $\left(y \cdot z_{p, \gamma}^{+}\right)_{p} \geq$ $U_{p, \gamma}-D \geq\left(x \cdot z_{p, \gamma}^{+}\right)_{p}$. Now by $\delta$-hyperbolicity we have

$$
(x \cdot y)_{p} \geq \min \left(\left(x \cdot z_{p, \gamma}^{+}\right)_{p},\left(y \cdot z_{p, \gamma}^{+}\right)_{p}\right)-\delta \geq\left(x \cdot z_{p, \gamma}^{+}\right)_{p}-\delta .
$$

By Lemma 1.13 we have that for all $x \notin O_{p}(\gamma, 0)$ and all $y \in O_{p}(\gamma, D)$, we have

$$
e^{\epsilon\left(x \cdot \gamma^{-1} p\right)_{p}} \leq \frac{e^{3 \epsilon \delta}}{d_{p}^{\epsilon}\left(x, z_{p, \gamma}^{+}\right)} \leq \frac{e^{4 \epsilon \delta}}{d_{p}^{\epsilon}(x, y)}
$$

Combining these estimates together, we obtain

$$
\begin{aligned}
e^{2 \alpha\left(x \cdot \gamma^{-1} p\right)_{p}} & =\frac{1}{\nu\left(O_{p}(\gamma, D)\right)} \int_{O_{p}(\gamma, D)} e^{2 \alpha\left(x \cdot \gamma^{-1} p\right)_{p}} d \nu(y) \\
& \leq \frac{1}{\nu\left(O_{p}(\gamma, D)\right)} \int_{O_{p}(\gamma, D)} \frac{e^{8 \alpha \delta}}{d_{p}^{\epsilon}(x, y)^{\frac{2 \alpha}{\epsilon}}} \nu(y)
\end{aligned}
$$

By setting $r=e^{-\epsilon\left(U_{p, \gamma}-D\right)}$ we obtain

$$
r^{\frac{\alpha}{\epsilon}} e^{2 \alpha\left(z_{p, \gamma}^{+} \cdot \gamma^{-1} p\right)_{p}} \geq e^{\alpha\left(U_{p, \gamma}+D\right)-2 \alpha \delta} \geq \frac{e^{\alpha D-2 \alpha \delta}}{\beta \nu\left(O_{p}(\gamma, D)\right)} .
$$

This implies the second condition, and therefore,

$$
\left(e^{2 \alpha\left(x \cdot \gamma^{-1} p\right)_{p}}, e^{-\epsilon\left(U_{p, \gamma}-D\right)}, z_{p, \gamma}, \frac{\alpha}{\epsilon}, \frac{\alpha}{\epsilon}, \max \left\{\beta e^{\alpha(10 \delta-D)}, e^{\alpha(8 \delta+2 D)}\right\}\right)
$$

is a spike for every $D \geq D_{0}$. For simplicity we note that

$$
\max \left\{\beta e^{\alpha(10 \delta-D)}, e^{\alpha(8 \delta+2 D)}\right\}<\beta e^{\alpha(10 \delta+2 D)},
$$

and so we use the right hand expression for the spike constant.

Lastly, to show we have a $\nu$-spike, observe that the local doubling constants are given by

$$
\frac{\nu\left(O_{p}(\gamma, D+\log (5) / \epsilon)\right)}{\nu\left(O_{p}(\gamma, D)\right)} \leq \beta^{2} e^{2 \alpha(D+\log (5) / \epsilon)} .
$$

This proves the lemma.

Corollary 7.2. Let $X$ be as in Proposition 7.1 and $\nu$ a nonatomic $\alpha$-quasiconformal measure with constant $C$. If $\nu^{\prime}=f \nu$ for a uniformly positive $f \in L^{\infty}(X, \nu)$, then there exists a constant $\beta \geq 1$ and $D_{0}>0$ such that for all $D \geq D_{0}$ and all $\epsilon>0$,

$$
\left(\frac{d\left(\gamma_{\star} \nu^{\prime}\right)}{d \nu}, e^{-\epsilon\left(U_{p, \gamma}-D\right)}, z_{p, \gamma}^{+}, \frac{\alpha}{\epsilon}, \frac{\alpha}{\epsilon}, C^{2} \beta e^{\alpha(10 \delta+2 D)}\|f\|_{L^{\infty}}\left\|\frac{1}{f}\right\|_{L^{\infty}}\right)
$$

is a $\nu$-spike on $\partial X$ with respect to the quasimetric $d_{p}^{\epsilon}$. 
Proof. Observe that

$$
\frac{d \gamma_{*} \nu^{\prime}}{d \nu}(x)=f\left(\gamma^{-1} x\right) \frac{d \gamma_{*} \nu}{d \nu}(x)
$$

and that for some $C \geq 1$,

$$
C^{-1} e^{2 \alpha\left(x \cdot \gamma^{-1} p\right)_{p}} \leq e^{\alpha d\left(p, \gamma^{-1} p\right)} \frac{d \gamma_{*} \nu}{d \nu}(x) \leq C e^{2 \alpha\left(x \cdot \gamma^{-1} p\right)_{p}} .
$$

Since $f$ is bounded from below and above we apply Lemma 4.5 twice to the previous proposition to obtain the result.

Proof of Theorem [0.1. Fix $p \in X$. Corollary 7.2 implies that the set of 6-tuples,

$$
\left\{\left(\frac{d \gamma_{\star} \nu}{d \nu}, e^{-\epsilon\left(U_{p, \gamma}-D\right)}, z_{p, \gamma}^{+}, \frac{\alpha}{\epsilon}, \frac{\alpha}{\epsilon}, C(D)\right)\right\}_{\gamma \in \Gamma, D \geq D_{0}},
$$

are a family of $\nu$-spikes where $C(D)=\beta e^{\alpha(10 \delta+2 D)}$. Observe that the balls $B_{d_{p}^{\epsilon}}\left(z_{p, \gamma}^{+}, e^{-\epsilon\left(U_{p, \gamma}-D\right)}\right)$ in the quasimetric $d_{p}^{\epsilon}$ are exactly $O_{p}(\gamma, D)$. Observe, that every Radon-Nykodym derivative appears once for each $D \geq D_{0}$. Noting that $U_{p, \gamma}$ is comparable to $d\left(p, \gamma^{-1} p\right)$, from the expression of Remark 1.25 we can write the radial limit set as

$$
\Lambda_{r}(\Gamma)=\bigcup_{D \geq D_{0}} \bigcap_{r>0} \bigcup_{\left\{\gamma \in \Gamma \mid e^{-\epsilon\left(U_{p}, \gamma-D\right)} \leq r\right\}} O_{p}(\gamma, D)
$$

Note that $C(D)$ and $D$ are monotone increasing with respect to each other. Write $D(\cdot)$ for the inverse function to $C(\cdot)$. In terms of the notation of Theorem [5.2 we have $B_{C}(r)=\bigcup_{\left\{\gamma \in \Gamma \mid e^{-\epsilon\left(U_{p}, \gamma-D\right)} \leq r\right\}} O_{p}(\gamma, D(C))$. Our assumption that the radial limit set has full measure within the limit set implies that $\lim _{C \rightarrow \infty} \nu\left(B_{C}\right)=1$. We also assumed that $\frac{d \gamma_{\star} \nu}{d \nu}$ is continuous for each $\gamma \in \Gamma$. We will apply Theorem 5.2 to this family of continuous spikes (normalized to be unit spikes), and we take $F=\frac{d \nu^{\prime}}{d \nu}$ which we assumed to be lower semicontinuous.

Before applying the Basis Theorem, we first need to know that $\nu$ has $\left(\frac{\alpha}{\epsilon}, \frac{\alpha}{\epsilon}\right)$ decay. Since $(X, \Gamma)$ is assumed to be quasiconvex cobounded, for any point $z \in \partial X$, there is a $C$-quasigeodesic ray ending in $z$ for some universal constant $C$. Therefore for any $r>0$, the family of shadows $\left\{O_{p}(\gamma, D)\right\}_{\gamma \in \Gamma}$ has a member containing $z$ and with $d_{p}^{\epsilon}$ radii bounded between $C r$ and $\frac{1}{C} r$ for some fixed $C>1$ and any sufficiently small $r>0$. Therefore the Shadow Lemma 1.26 implies that $\nu$ is upper and lower $\frac{\alpha}{\epsilon}$-regular with respect to $d_{p}^{\epsilon}$. Hence Lemma 3.3 implies the decay property. Incidentally, the lower regularity gives us the strong doubling property for $\nu$, though we did not need this.

Similarly, when $\nu=\nu_{p}$ belongs to a bounded density $\left\{\nu_{q}\right\}_{q \in X}$, case b') of the Shadow Lemma together with the assumption on $X$ implies the upper regularity for shadows $\left\{O_{p}(q, D)\right\}_{q \in X}$ which form a cover of $\Lambda_{r}$ with radii bounded between $C r$ and $\frac{1}{C} r$ for some fixed $C>1$ and any sufficiently small $r>0$. This implies the upper Ahlfors regularity for $\nu$ on $\Lambda_{r}$ without the quasiconvex cobounded assumption on $\Gamma$. In particular, $\nu$ has $\left(\frac{\alpha}{\epsilon}, \frac{\alpha}{\epsilon}\right)$ decay. 
Theorem 0.1 now follows from the Basis Theorem by taking $\mu(\gamma)=\lambda_{\gamma}$, since for every measurable set $E$ we have

$$
\mu \star \nu(E)=\int_{E} \sum_{\gamma \in \Gamma} \mu(\gamma) \frac{d \gamma_{\star} \nu}{d \nu} d \nu=\int_{E} \frac{d \nu^{\prime}}{d \nu} d \nu=\nu^{\prime}(E)
$$

Proof of Corollary 0.3. By the proximality criterion established in Fur73 we need to show that for each $x \in \Lambda$, there is a sequence $\left(\gamma_{i}\right) \subset \Gamma$ such that $\gamma_{i *} \nu$ converges weakly to an atomic measure (of any positive weight) at $x$. Since $x \in \Lambda$ there is a sequence $\left(\gamma_{i}\right)$ such that for the base point $p \in X, \gamma_{i} p$ tends to $x$. In particular, $\left(\gamma_{i}^{-1} p \cdot x\right)_{p}$ and $d\left(p, \gamma_{i} p\right)$ both tend to $\infty$ as $i$ does. Since $\nu$ is bounded $\alpha$-quasiconformal, $\int_{\Lambda} d \gamma_{i_{*}} \nu \geq C$ independently of $i$. On the other hand for any open neighborhood $\mathcal{O} \subset \Lambda$ of $x$, we have

$$
\int_{\Lambda \backslash \mathcal{O}} d \gamma_{i_{*}} \nu=\int_{\Lambda \backslash \mathcal{O}} \frac{d \gamma_{i_{*}} \nu}{d \nu}(z) d \nu(z) \leq \int_{\Lambda \backslash \mathcal{O}} C e^{-\alpha \rho_{p, z}\left(\gamma_{i}^{-1} p\right)} d \nu(z)
$$

which vanishes as $i$ tends to $\infty$ since $\left(z \cdot \gamma_{i}^{-1} p\right)_{p}$ remains bounded for $z \notin \mathcal{O}$ while $d\left(p, \gamma_{i}^{-1} p\right)$ becomes uniformly large. Therefore, $\left\{\gamma_{*} \nu\right\}_{\gamma \in \Gamma}$ forms a proximal family on $\Lambda$.

\section{RADON-NIKODYM DERIVATIVES AS $Q$-SPIKES}

In this section we prove Theorem 0.4 . We assume that $(H, d)$ is a $\mathrm{CAT}(-1)$ space and that $\nu$ is a Lipschitz $\alpha$-quasiconformal measure on $\Lambda \subset \partial H$ for any $\alpha>0$ for which one exists. In order to avoid treating separate cases in the analysis below, we will also assume $\epsilon \leq \alpha$.

For each $\gamma \in \Gamma$ we let $f_{\gamma}(z)=\frac{d \gamma_{\star} \nu}{d \nu}(z)$. Recall that $U_{p, \gamma}=d\left(p, \gamma^{-1} p\right)$ and set $z_{p, \gamma}^{+}$to be the end of a geodesic starting at $p$ and passing through $\gamma^{-1} p$. In this section we will show the following proposition.

Proposition 8.1. For each $p \in H$ and $\gamma \in \Gamma$, the tuple

$$
\left(e^{2 \alpha\left(x \cdot \gamma^{-1} p\right)_{p}}, e^{-\epsilon\left(d\left(p, \gamma^{-1} p\right)-D\right)}, z_{p, \gamma}^{+}, \frac{\alpha}{\epsilon}, \frac{\alpha}{\epsilon}, C(\alpha, \epsilon, D)\right)
$$

is a $Q$-spike for $Q=\frac{\alpha}{\epsilon}$, where $C(\alpha, \epsilon, D)$ only depends on $\alpha, \epsilon$ and $D$.

We will need the following lemmas.

Lemma 8.2. Let $\rho, \rho^{\prime}$ be two metrics on $\partial H$. Let $x \in \partial H$. Define $f(z)=\frac{\rho^{\prime}(x, z)}{\rho(x, z)}$.

Then for any $z, y \neq x$ we have

$$
\frac{f(z)-f(y)}{\rho(z, y)} \leq \frac{f(z)}{\rho(x, y)}+\frac{\rho^{\prime}(z, y)}{\rho(z, y)} \frac{1}{\rho(x, y)}
$$


Proof.

$$
\begin{aligned}
\frac{|f(z)-f(y)|}{\rho(z, y)} & =\frac{\mid \frac{\rho^{\prime}(x, z)}{\rho(x, z)}-\frac{\rho^{\prime}(x, y) \mid}{\rho(x, y)}}{\rho(z, y)}=\frac{\left|\rho^{\prime}(x, z) \rho(x, y)-\rho^{\prime}(x, y) \rho(x, z)\right|}{\rho(z, y) \rho(x, z) \rho(x, y)}= \\
& =\frac{\rho^{\prime}(x, z)|\rho(x, y)-\rho(x, z)|+\left|\rho^{\prime}(x, z)-\rho^{\prime}(x, y)\right| \rho(x, z)}{\rho(z, y) \rho(x, z) \rho(x, y)}= \\
& =\frac{f(z)}{\rho(x, y)} \frac{|\rho(x, y)-\rho(x, z)|}{\rho(z, y)}+\frac{\left|\rho^{\prime}(x, z)-\rho^{\prime}(x, y)\right|}{\rho(z, y)} \frac{1}{\rho(x, y)}
\end{aligned}
$$

By triangle inequality we have $|\rho(x, y)-\rho(x, z)| \leq \rho(y, z)$ and $\mid \rho^{\prime}(x, z)-$ $\rho^{\prime}(x, y) \mid \leq \rho^{\prime}(z, y)$

Substituting we obtained required inequality.

Now we apply this lemma to our Radon-Nikodym derivatives. The following can be viewed as a quantitative version of the Lipschitz property for Busemann functions established by Bourdon [Bou96. Perhaps this can be derived directly from that result.

Lemma 8.3. For $p, q \in H$, fix $\epsilon$ such that $d_{p}^{\epsilon}$ and $d_{q}^{\epsilon}$ are metrics. Then

$$
\frac{\left|e^{\epsilon(z \cdot q)_{p}}-e^{\epsilon(y \cdot q)_{p}}\right|}{d_{p}^{\epsilon}(y, z)} \leq 4 e^{\epsilon d(p, q)} \frac{e^{\epsilon(z \cdot q)_{p}}}{\operatorname{diam}(\Lambda)}
$$

for any $y, z \in \partial H$.

Proof. Fix $x \in \partial H$ such that $d_{p}^{\epsilon}(y, x) \geq \frac{\operatorname{diam}(\Lambda)}{2}$. Let

$$
f(w)=\frac{d_{q}^{\epsilon}(w, x)}{d_{p}^{\epsilon}(w, x)} .
$$

By Lemma 1.14 we have

$$
\frac{d_{q}^{\epsilon}(z, y)}{d_{p}^{\epsilon}(z, y)} \leq f(z) e^{\epsilon\left((y \cdot q)_{p}-(x \cdot q)_{p}\right)} \leq f(z) e^{\epsilon d(p, q)}
$$

By Lemma 8.2 we have

$$
\frac{|f(z)-f(y)|}{d_{p}^{\epsilon}(z, y)} \leq \frac{f(z)}{d_{p}^{\epsilon}(x, y)}+\frac{d_{q}^{\epsilon}(z, y)}{d_{p}^{\epsilon}(z, y)} \frac{1}{d_{p}^{\epsilon}(x, y)} \leq \frac{4 f(z) e^{\epsilon d(p, q)}}{\operatorname{diam}(\Lambda)} .
$$

Now use the fact that

$$
f(w)=e^{\epsilon(w \cdot q)_{p}} e^{\epsilon(x \cdot q)_{p}},
$$

to eliminate $f$ everywhere in the above formula. To finish the proof divide everything by $e^{\epsilon(x \cdot q)_{p}}$.

Proof of Proposition 8.1. Let $D_{0}$ be the constant from Lemma 1.26 Fix any $D \geq$ $D_{0}$, so that by Proposition 7.1

$$
\left(e^{2 \alpha\left(x \cdot \gamma^{-1} p\right)_{p}}, e^{-\epsilon\left(d\left(p, \gamma^{-1} p\right)-D\right)}, z_{p, \gamma}^{+}, \frac{\alpha}{\epsilon}, \frac{\alpha}{\epsilon}, C(\alpha, D)\right)
$$

is a $\nu$-spike for $C(\alpha, D)=\beta e^{\alpha(10 \delta+2 D)}$. Set $q=\gamma^{-1} p$ and $r=e^{-\epsilon(d(p, q)-D)}$. 
Now apply the inequality $\left|x^{k}-y^{k}\right| \leq k|x-y| \max (x, y)^{k-1}$ for all $k \geq 1$ to the Lemma 8.3 to obtain

$$
\begin{aligned}
\frac{\left|e^{\alpha(z \cdot q)_{p}}-e^{\alpha(y \cdot q)_{p}}\right|}{d_{p}^{\epsilon}(y, z)} & \leq \frac{\alpha}{\epsilon} \frac{\left|e^{\epsilon(z \cdot q)_{p}}-e^{\epsilon(y \cdot q)_{p}}\right|}{d_{p}^{\epsilon}(y, z)} \max \left(e^{\epsilon(z \cdot q)_{p}}, e^{\epsilon(y \cdot q)_{p}}\right)^{\frac{\alpha}{\epsilon}-1} \leq \\
& \leq 4 \frac{\alpha}{\epsilon} e^{\epsilon d(p, q)} \frac{e^{\epsilon(z \cdot q)_{p}}}{\operatorname{diam}(\Lambda)} \max \left(e^{\epsilon(z \cdot q)_{p}}, e^{\epsilon(y \cdot q)_{p}}\right)^{\frac{\alpha}{\epsilon}-1} .
\end{aligned}
$$

From the definition of $\nu$-spike we have that $e^{\alpha(z \cdot q)_{p}} \leq C(\alpha, D) e^{\alpha(y \cdot q)_{p}}$ for all $z, y$ such that $d_{p}^{\epsilon}(z, y) \leq e^{-\epsilon\left(d\left(p, \gamma^{-1} p\right)-D\right)}$. This implies that

$$
\begin{aligned}
D_{r} e^{2 \alpha(y \cdot q)_{p}} & \leq 4 \frac{\alpha}{\epsilon} e^{\epsilon d(p, q)} \frac{e^{\alpha(y \cdot q)_{p}}}{\operatorname{diam}(\Lambda)} C(\alpha, D)^{\frac{\alpha-\epsilon}{\alpha}}= \\
& =\frac{4 \alpha C(\alpha, D)^{\frac{\alpha-\epsilon}{\alpha}} e^{\alpha D}}{\epsilon \operatorname{diam}(\Lambda)} \frac{e^{2 \alpha(y \cdot q)_{p}}}{r} .
\end{aligned}
$$

However, we assumed $\nu$ was $\alpha$-quasiconformal, so the Shadow Lemma 1.26 shows

$$
\nu\left(B\left(z_{p, \gamma}^{+}, e^{-\epsilon(d(p, q)-D)}\right)\right) \geq \beta e^{\alpha d(p, q)}=\beta r^{\frac{\alpha}{\epsilon}} e^{-\alpha D} .
$$

Setting $C(\alpha, \epsilon, D)=\max \left(\frac{4 \alpha C(\alpha, D)^{\frac{\alpha-\epsilon}{\alpha}} e^{\alpha D}}{\epsilon \operatorname{diam}(\Lambda)}, C(\alpha, D), \beta e^{\alpha D}\right)$ we finish the proof.

Proof of Theorem 0.4. Since $\nu$ is Lipschitz $\alpha$-quasiconformal, and in light of Lemma 3.8 each Radon-Nikodym derivative $\frac{d \gamma_{\star} \nu}{d \nu}$ can be expressed as

$$
\frac{d \gamma_{\star} \nu}{d \nu}(z)=R_{\gamma}(z) e^{2 \alpha\left(\gamma^{-1} p \cdot z\right)_{p}} e^{-\alpha d\left(p, \gamma^{-1} p\right)}
$$

for a Lipschitz function $R_{\gamma}$ on $\Lambda$ satisfying $K^{-1} \leq R_{\gamma} \leq K$ and $D_{r} R_{\gamma} \leq \frac{K}{r}$, for a constant $K \geq 1$ independent of $\gamma \in \Gamma$ and all $r>0$. Hence by Lemma 4.9 the tuples

$$
\left(\frac{d \gamma_{\star} \nu}{d \nu}, e^{-\epsilon\left(d\left(p, \gamma^{-1} p\right)-D\right)}, z_{p, \gamma}^{+}, \frac{\alpha}{\epsilon}, \frac{\alpha}{\epsilon}, K^{2} C(\alpha, \epsilon, D)\right)
$$

also form a family of $\frac{\alpha}{\epsilon}$-spikes indexed by $\gamma \in \Gamma$ and all $D \geq D_{0}$.

We assumed that $\operatorname{diam}(X / \Gamma)<\infty$, so we may set $D=\max \left\{\operatorname{diam}(X / \Gamma), D_{0}\right\}$. Hence, for every $R>0$, the union of shadows $\bigcup_{\left\{\gamma \mid R-D \leq d\left(p, \gamma^{-1} p\right) \leq R\right\}} O_{p}(\gamma, D)$ covers $\Lambda$. We assume that this was the choice of $D$ taken in the proof of Proposition 8.1 above. In the notation of Theorem 6.1 this means that we can take $g(r)=e^{-\epsilon D} r$ and that $B_{C}=\Lambda$ where $C=K^{2} C(\alpha, \epsilon, D)$.

We now apply case 3 ) of Theorem 6.1 to these spike and set the function $F=1$. Setting $\mu(\gamma)=\lambda_{\gamma}$, then as in the proof of Theorem 0.1 we have $\mu \star \nu=\nu$. Since $\mu$ also has finite first moment, we apply the criteria of Kaimanovich to conclude that $(\Lambda, \nu)$ is a Poisson boundary for $\mu$.

Remark 8.4. We could have weakened the hypothesis on $\Gamma$ in Theorem 0.4 so that

$$
B_{C}=\bigcap_{r>0} \bigcup_{\left\{\gamma \mid e^{-\epsilon d\left(p, \gamma^{-1} p\right)}<r\right\}} O_{p}(\gamma, D(C))
$$

satisfies either the assumptions of case 1) or 2) in Theorem 6.1 However, there does not seem to be a simple intrinsic condition on $\Gamma$ which guarantees this. 
Corollary 8.5. In Corollary 0.3 and Theorem 0.4 the space of measures $\mu$ for which a given measure $\nu$ on $\partial X$ is stationary (respectively, a Poisson Boundary) is infinite dimensional. Moreover, if $\eta$ is any finite Borel measure on $\Gamma$ then the measure $\mu$ can be chosen so that $\eta$ is absolutely continuous with respect to $\mu$. If $\eta$ has full support on $\Gamma$, then $\mu$ can be chosen in the same class as $\eta$.

Proof. if we started with any finite sum $\sum_{i=1}^{k} \lambda_{i} f_{i}<F$ with $f_{i}=\frac{d \gamma_{i} \nu}{d \nu}$ for any choice of $\gamma_{i} \in \Gamma$, then we could apply Theorem 6.1 or 5.2 to write $F-\sum_{i=1}^{k} \lambda_{i} f_{i}$ in a basis that excluded $f_{1}, \ldots, f_{k}$. In other words, for any finite set $\left\{\gamma_{1}, \ldots, \gamma_{k}\right\} \subset \Gamma$, we can specify the value of $\mu\left(\gamma_{i}\right)$ within an interval $\left[0, \epsilon_{i}\right]$ for some $\epsilon_{i}>0$. In particular, the space of measures for which $\nu$ is stationary, or a Poisson boundary measure, is always infinite dimensional.

For the second statement, recall that $f_{\gamma}(x)=\frac{d \gamma_{*} \nu}{d \nu}(x)$ are bounded continuous functions on $\Lambda$. We can choose a positive function $\eta$-measureable $w$ on $\Gamma$ so that $w(\gamma) \leq \frac{1}{\sup _{x \in \Lambda} f_{\gamma}(x)}$ for all $\gamma \in \Gamma$. For each $x \in \Lambda, f_{\gamma}(x)$ is continuous in $\gamma$ with respect to the topology on $\Gamma$ inherited as a subspace of $\operatorname{Isom}(X)$ endowed with the compact open topology. Since $\eta$ is Borel, it follows that $w(\gamma) f_{\gamma}(x)$ is $\eta$-measurable for all $x \in \Lambda$. By Lebesgue dominated convergence, $\int_{\Gamma} w(\gamma) f_{\gamma} d \eta(\gamma)$ is continuous on all of $\Lambda$. By dividing $w$ by a fixed constant we may assume $\int_{\Gamma} w(\gamma) f_{\gamma} d \eta(\gamma)$ is less than 1 or $\frac{d \nu^{\prime}}{d \nu}$, whichever is the case.

Now we repeat the proof of the theorems by approximating a new function. For instance, in the first theorem we approximate $\frac{d \nu^{\prime}}{d \nu}-\int_{\Gamma} w(\gamma) f_{\gamma} d \eta(\gamma)$ in place of $\frac{d \nu^{\prime}}{d \nu}$. In either case, this results in a measure $\mu^{\prime}$. We finally set $\mu=\mu^{\prime}+w \eta$.

For the last statement, suppose we have satisfied the hypotheses of Corollary 0.3 (respectively Theorem 0.4) so that we have found a subset $S \subset \Gamma$ such that $\left\{\frac{d \gamma_{*} \nu}{d \nu}\right\}_{\gamma \in S}$ form a family of $\nu$-spikes (resp. $Q$-spikes) satisfying the hypotheses of Theorem 5.2 (resp. Theorem [6.1). For each $\gamma \in S$, we wish to replace the continuous $\nu$-spikes (resp. $Q$-spikes) corresponding to the functions $\frac{d \gamma_{*} \nu}{d \nu}$ on $\Lambda$ with the functions $f_{\gamma}$ given by

$$
f_{\gamma}(x)=\int_{\Gamma} \phi_{\gamma}\left(\gamma^{\prime}\right) \frac{d \gamma_{*}^{\prime} \nu}{d \nu}(x) d \eta\left(\gamma^{\prime}\right)
$$

where $\phi_{\gamma}$ is a yet to be chosen bounded Borel measurable function on $\Gamma$. We have assumed that the support of $\eta$ contains an open neighborhood of $S$. Hence for each $\gamma \in S$, there is a sequence $\left(\theta_{\gamma, i}\right)$ of bounded continuous functions on $\Gamma$ such that $\theta_{\gamma, i} \eta$ converges weakly to the unit atomic measure at $\gamma$. Since the $\frac{d \gamma_{*} \nu}{d \nu}(x)$ are continuous $\nu$-spikes (resp. $Q$-spikes for $Q=\frac{\alpha}{\epsilon}$ ), the Lebesgue dominated convergence theorem implies that if $\phi_{\gamma}$ is chosen to be $\theta_{\gamma, i}$ for some sufficiently large $i=i(\gamma)$, then $f_{\gamma}$ will still satisfy the continuous $\nu$-spike (resp. $Q$-spike with $Q=\frac{\alpha}{\epsilon}$ ) conditions, but with each spike constant $C_{\gamma}$ for $\frac{d \gamma_{*} \nu}{d \nu}(x)$ uniformly enlarged to, for instance, $2 C_{\gamma}$ for $f_{\gamma}$. Applying the proofs of Theorems 0.1 and 0.4 to the new spikes $f_{\gamma}$ yields a finite measure $\mu$ of the form $\mu=\sum_{i=1}^{\infty} \lambda\left(\gamma_{i}\right) \phi_{\gamma_{i}} \eta$. Consequently, $\mu$ is absolutely continuous with respect to $\eta$, though not necessarily conversely. However, by the penultimate argument, we may augment $\mu$ to be the same measure class as $\eta$.

Remark 8.6. In all of our constructions of stationizing measures $\mu$, the support must at least contain a countably infinite subset of $\Gamma$. However, as we will see in 
the next section, there are special cases of $\Gamma$ and $(\partial \Gamma, \nu)$ for which the support of $\mu$ can be finite. However, these do not arise from our construction directly.

We end this section with the proof of the last corollary of the introduction.

Proof of Corollary 0.5. We first show that the Hausdorff measures and the PattersonSullivan measures, assuming they exist for the given $\Gamma$ action on $H$, are Lipschitz $\alpha$-quasiconformal measures. By Proposition 2.6 and Corollary 2.1] we know that these measures are $\alpha$-conformal measures. However, Proposition 8.3 shows that the Gromov product, and consequently Busemann functions, are Lipschitz on the boundary of CAT(-1) spaces. Hence these measures have Lipschitz Radon-Nikodym derivatives with respect to the $\Gamma$ transformations.

If $m$ is either such a Hausdorff or Patterson-Sullivan measure and $\nu=f m$ for a Lipschitz $f$, then we have

$$
\frac{d \gamma_{*} \nu}{d \nu}(x)=\frac{d \gamma_{*} f m}{d f m}(x)=\frac{f\left(\gamma^{-1} x\right)}{f(x)} \frac{d \gamma_{*} m}{d m} .
$$

We assumed $\frac{1}{K} \leq f<K$ and $D_{r} f<K$ for some $K \geq 1$ and all $r$ less than some fixed value $C$. On the other hand, we have by Lemma 3.7

$$
D_{r}\left(f \circ \gamma^{-1}\right)(x) \leq D_{r D_{r}\left(\gamma^{-1}\right)(x)}(f)\left(\gamma^{-1} x\right) D_{r}\left(\gamma^{-1}\right)(x) .
$$

However, we only need to estimate this for $r=e^{-\epsilon d\left(p, \gamma^{-1} p\right)}$. Since $D_{r}\left(\gamma^{-1}\right)(x)<$ $C e^{\epsilon d\left(p, \gamma^{-1} p\right)}$ for some $C>0$ we obtain $D_{r}\left(f \circ \gamma^{-1}\right)(x)<\frac{K C}{r}$. Hence by Lemma $3.7 D_{r} \frac{f\left(\gamma^{-1}\right)}{f}<\frac{K^{3} C}{r}$. Lemma 4.9 implies that $\frac{d \gamma_{*} \nu}{d \nu}$ are $Q$-spikes with spike constant $2 K^{5} \mathrm{C}$ which is independent of $\gamma$. This allows us to employ the rest of the proof of Theorem 0.4

\section{Application to the Free Groups}

Here we present an application of Theorem 6.1 to the case of a free group $\Gamma$. It is easy to verify that the Cayley graph of $\Gamma$, equipped with any metric quasi-isometric to the word metric, is a Gromov hyperbolic space. With even less effort one may check that its Cayley graph for any set of generators is a CAT $(-\kappa)$ space for any $\kappa \leq 0$.

We will prove that the (class of) Patterson-Sullivan measures is harmonic, i.e., there exists a random walk on the free group such that the induced measure on the boundary is in the maximal Hausdorff class.

We show that normalized Radon-Nikodym derivative satisfy Theorem 6.1

Let $X$ be a Cayley graph for a free group $\Gamma$ with standard set of generators (i.e. generators without any relations) and any positive weights on the graph. Let $d$ be the corresponding weighted path metric.

Let $x, y \in X$ and $z \in \partial X$. Let $z_{o} \in X$ be a point on the segment $[x, y]$ and geodesic connecting $x$ and $z$ such that the $d\left(x, z_{o}\right)$ achieves the maximum. Then the Busemann function can be represented as,

$$
\rho_{x, z}(y)=d\left(x, z_{o}\right)-d\left(y, z_{o}\right) .
$$

Therefore for $z, o \in \partial X$. Let $z \in X$ be a point on the geodesic $[z, o]$ connecting $z$ and $o$ which is closest to $x$. Then

$$
d_{x}^{\epsilon}(z, o)=e^{-\epsilon d(x, y)} .
$$


Let $\alpha / \epsilon$ be the Hausdorff dimension for the metric $d_{p}^{\epsilon}$ and $\nu$ be the corresponding $\alpha$-conformal density, which coincides with the Patterson-Sullivan measure at $p$. Then we have for $p=\mathbf{e}$ and all $\gamma \in \Gamma$

$$
\frac{d \gamma_{\star} \nu}{d \nu}(z)=e^{-\alpha \rho_{\gamma-1}, z}(p)
$$

So since $-d\left(p, \gamma^{-1}(p)\right) \leq \rho_{\gamma^{-1} p, z}(p) \leq d\left(p, \gamma^{-1}(p)\right)=\|\gamma\|$, we have that

$$
e^{-\alpha\|\gamma\|} \leq \frac{d \gamma_{\star} \nu}{d \nu}(z) \leq e^{\alpha\|\gamma\|}
$$

Let $f_{\gamma}(z)=\frac{d \gamma_{\star} \nu}{d \nu}(z)$. It is not difficult to check that $\left(f_{\gamma}(z), z_{\gamma}, e^{-\epsilon\|\gamma\|}, \alpha, \alpha, e^{-1}\right)$ is a spike, where $z_{\gamma}$ is the endpoint of any geodesic which starts at $p$ and passes through $\gamma^{-1} p$.

Now let us estimate $D_{e^{-\epsilon\|\gamma\|}} f_{\gamma}(z)$.

Lemma 9.1. For any $q, p \in X$, the Busemann function $\rho_{q, .}(p)$ is locally constant on $\partial X$ taking on only a finite number of values. Furthermore, $D_{e^{-\epsilon}\|\gamma\|} f_{\gamma}(z)=0$ for all $\gamma \in \Gamma$.

Proof. Let $o, z \in \partial X$ be such that $d_{p}^{\epsilon}(o, z)<e^{-\epsilon d(p, q)}$. In particular, the point $y$ lying on the geodesic $[o, z]$ and closest to $p$ does not lie on the segment $[p, q]$.

Let $x$ be the point of the segment $[p, q]$ closest to $y$. Now since $y \neq x$, we have that

$$
\rho_{q, z}(p)=d(y, q)-d(y, p)=d(x, q)-d(x, p)
$$

on the other hand,

$$
\rho_{q, o}(p)=d(y, q)-d(y, p)=d(x, q)-d(x, p) .
$$

So $\rho_{q, z}(p)=\rho_{q, o}(p)$. The first claim follows from the fact that a finite number of $d_{p}$ balls with radius $e^{-\epsilon d(p, q)}$ cover $\partial X$.

For the second statement, let $o, z \in \partial X$ be such that $d_{p}^{\epsilon}(o, z)<e^{-\epsilon\|\gamma\|}$. Since $\|\gamma\|=d\left(\gamma^{-1} p, p\right)$, we have $\rho_{\gamma^{-1} p, z}(p)=\rho_{\gamma^{-1} p, o}(p)$ or $f_{\gamma}(z)=f_{\gamma}(o)$.

It is clear that the ball of the radius $e^{-\epsilon n}$ form a Vitally Covering for any $n \in \mathbb{N}$. Therefore, we can apply Theorem 6.1 to obtain that

$$
1=\sum_{\gamma \in \Gamma} \lambda_{\gamma} f_{\gamma}(z)
$$

or equivalently

$$
\nu=\sum_{\gamma \in \Gamma}\left(\lambda_{\gamma}\left\|f_{\gamma}\right\|_{L^{1}}\right) \gamma_{\star} \nu .
$$

with the property that

$$
-\sum_{\gamma \in \Gamma}\left(\lambda_{\gamma}\left\|f_{\gamma}\right\|_{L^{1}}\right) \log \left(\left\|f_{\gamma}\right\|_{L^{1}}\right)=\sum_{\gamma \in \Gamma} \lambda_{\gamma}\left\|f_{\gamma}\right\|_{L^{1}}(\epsilon\|\gamma\|)<\infty .
$$

Hence if $\mu(\gamma)=\lambda_{\gamma}\left\|f_{\gamma}\right\|_{L^{1}}$, then $\mu$ is a probability measure with finite first moment and $\nu$ is $\mu$-stationary.

In the case when the edge weights only depend on the reduced word distance to the identity, the measure $\mu$ can be represented very simply. If $\mu$ is constant on a single sphere about the identity, $\|\gamma\|=k$, then it is easy to compute using Lemma 9.1 that $\mu \star \nu=\nu$. More generally, any measure which is constant on spheres has this property since the average of two measures which stationize $\nu$ also stationizes 
$\nu$. Although from Corollary 8.5 we see that these are only the most symmetric of the measures which stationize $\nu$.

\section{REFERENCES}

[BH99] M. R. Bridson and A. Haefliger, Metric spaces of non-positive curvature, Grundlehren der Mathematischen Wissenschaften [Fundamental Principles of Mathematical Sciences], vol. 319, Springer-Verlag, Berlin, 1999. MR 2000k:53038

[Bou95] M. Bourdon, Structure conforme au bord et flot géodésique d'un cat(-1)-espace, Enseign. Math. (2) 41 (1995), no. 1-2, 63-102. MR 96f:58120

[Bou96] Sur le birapport au bord des cat $(-1)$-espaces, Inst. Hautes Études Sci. Publ. Math. (1996), no. 83, 95-104. MR 97k:58123

[Bow] B. Bowditch, Relatively hyperbolic groups, Preprint.

[BP00] M. Bourdon and H. Pajot, Rigidity of quasi-isometries for some hyperbolic buildings, Comment. Math. Helv. 75 (2000), no. 4, 701-736. MR 1789183

[BS00] M. Bonk and O. Schramm, Embeddings of Gromov hyperbolic spaces, Geom. Funct. Anal. 10 (2000), no. 2, 266-306. MR 2001g:53077

[BW89] F. F. Bonsall and D. Walsh, Vanishing $l^{1}$-sums of the Poisson kernel, and sums with positive coefficients, Proc. Edinburgh Math. Soc. (2) 32 (1989), no. 3, 431-447. MR 90m:31001

[CLSW98] F. H. Clarke, Yu. S. Ledyaev, R. J. Stern, and P. R. Wolenski, Nonsmooth analysis and control theory, Graduate Texts in Mathematics, vol. 178, Springer-Verlag, New York, 1998. MR 99a:49001

[CLW95] F. H. Clarke, Yu. S. Ledyaev, and P. R. Wolenski, Proximal analysis and minimization principles, J. Math. Anal. Appl. 196 (1995), no. 2, 722-735. MR 96i:49028

[Coo93] M. Coornaert, Mesures de Patterson-Sullivan sur le bord d'un espace hyperbolique au sens de Gromov, Pacific J. Math. 159 (1993), no. 2, 241-270. MR 94m:57075

[Fed69] H. Federer, Geometric measure theory, Springer-Verlag New York Inc., New York, 1969.

[Fur63] H. Furstenberg, A Poisson formula for semi-simple Lie groups, Ann. of Math. (2) 77 (1963), 335-386. MR 26 \#3820

[Fur67] $\quad$ Poisson boundaries and envelopes of discrete groups, Bull. Amer. Math. Soc. 73 (1967), 350-356. MR 35 \#1698

[Fur71] _ Random walks and discrete subgroups of Lie groups, Advances in Probability and Related Topics, Vol. 1, Dekker, New York, 1971, pp. 1-63. MR 44 \#1794

[Fur73] B Boundary theory and stochastic processes on homogeneous spaces, Harmonic analysis on homogeneous spaces (Proc. Sympos. Pure Math., Vol. XXVI, Williams Coll., Williamstown, Mass., 1972), Amer. Math. Soc., Providence, R.I., 1973, pp. 193229. MR $50 \# 4815$

[Gar96] S. J. Gardiner, Representation of continuous functions as sums of Green functions, Proc. Amer. Math. Soc. 124 (1996), no. 4, 1149-1157. MR 96g:31005

[GdlH90] É. Ghys and P. de la Harpe, Sur les groupes hyperboliques d'après mikhael gromov (bern, 1988), Progr. Math., vol. 83, Birkhäuser Boston, Boston, MA, 1990. MR 1086 649

[Gro87] M. Gromov, Hyperbolic groups, Essays in group theory, Springer, New York, 1987, pp. 75-263. MR 89e:20070

[Hae91] A. Haefliger, Complexes of groups and orbihedra, Group theory from a geometrical viewpoint (Trieste, 1990), World Sci. Publishing, River Edge, NJ, 1991, pp. 504-540. MR 93m:20048

[HL90] W. K. Hayman and T. J. Lyons, Bases for positive continuous functions, J. London Math. Soc. (2) 42 (1990), no. 2, 292-308. MR 92a:31002

[Kai90] V. A. Kaimanovich, Invariant measures of the geodesic flow and measures at infinity on negatively curved manifolds, Ann. Inst. Henri Poincaré Physique Théorique 53 (1990), no. 4, 361-393.

[Kai00] The Poisson formula for groups with hyperbolic properties, Ann. of Math. (2) 152 (2000), no. 3, 659-692. MR 2002d:60064

[Kai03] _ Poisson boundary of discrete groups, Unpublished Survey available at http://name.math.univ-rennes1.fr/vadim.kaimanovich/ 2003. 
[KV83] V. A. Kaimanovich and A. M. Vershik, Random walks on discrete groups: boundary and entropy, Ann. Probab. 11 (1983), no. 3, 457-490. MR 85d:60024

[Mic01] R. Miculescu, Approximation of continuous functions by Lipschitz functions, Real Anal. Exchange 26 (2000/01), no. 1, 449-452. MR 2002c:26004

[Pat76] S. J. Patterson, The limit set of a Fuchsian group, Acta Math. 136 (1976), no. 3-4, 241-273.

[Sul79] D. Sullivan, The density at infinity of a discrete group of hyperbolic motions, Inst. Hautes Études Sci. Publ. Math. (1979), no. 50, 171-202. MR 81b:58031

[Ton52] H. Tong, Some characterizations of normal and perfectly normal spaces, Duke Math. J. 19 (1952), 289-292. MR 14,304b

[Yue96] C. B. Yue, The ergodic theory of discrete isometry groups on manifolds of variable negative curvature, Trans. Amer. Math. Soc. 348 (1996), no. 12, 4965-5005.

INDIANA UNIVERSITY

E-mail address: cconnell@math.indiana.edu

UNIVERSITY OF CHICAGO

E-mail address: roma@math.uchicago.edu 